\title{
HTGR SPENT FUEL COMPOSITION AND FUEL ELEMENT BLOCK FLOW
}

\author{
by \\ C. J. HAMILTON, N. D. HOLDER, \\ V. H. PIERCE, and \\ M. W. ROBERTSON
}

This report was preporice

sponsored by the Urepared as an account of work Re United States nor the Uinted States Nethe they enployelopment Administration, nor any of subcontractors, or thes any of thesr contractors. warranty, express or impled oloyees, makes any

trabuluy or responsabuty for the or assumes any legal

or usefulness of any information, appey. Completeness process disclosed, or represents that its use product or

Prepared under

Contract E(04-3)-167

Project Agreement No. 53

for the San Francisco Operations Office

U.S. Energy Research and Development Administration

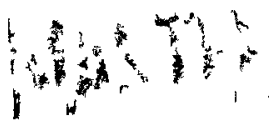




\section{DISCLAIMER}

This report was prepared as an account of work sponsored by an agency of the United States Government. Neither the United States Government nor any agency Thereof, nor any of their employees, makes any warranty, express or implied, or assumes any legal liability or responsibility for the accuracy, completeness, or usefulness of any information, apparatus, product, or process disclosed, or represents that its use would not infringe privately owned rights. Reference herein to any specific commercial product, process, or service by trade name, trademark, manufacturer, or otherwise does not necessarily constitute or imply its endorsement, recommendation, or favoring by the United States Government or any agency thereof. The views and opinions of authors expressed herein do not necessarily state or reflect those of the United States Government or any agency thereof. 


\section{DISCLAIMER}

Portions of this document may be illegible in electronic image products. Images are produced from the best available original document. 
$\bullet$ 
ABSTRACT

The High-Temperature Gas-Cooled Reactor (HTGR) utilizes the thoriumuranium fuel cycle. Fully enriched uranium fissile material and thorium fertile material are used in the initial reactor core and for makeup fuel in the recycle core loadings. Bred U-233 and unburned U-235 fissile materials are recovered from spent fuel elements, refabricated into recycle fuel elements, and used as part of the recycle core loading along with the makeup fuel elements. A typical HTGR employs a 4-yr fuel cycle with approximately one-fourth of the core discharged and reloaded annually.

The fuel element composition, including heavy metals, impurity nuclides, fission products, and activation products, has been calculated for discharged spent fuel elements and for reload fresh fuel and recycle fuel elements for each cycle over the life of a typical HTGR. Fuel element compositions are presented for the conditions of equilibrium recycle. Data describing compositions for individual reloads throughout the reactor life are available in a detailed volume upon request.

Fuel element block flow data have been compiled based on a forecast HTGR market. Annual block flows are presented for each type of fuel element discharged from the reactors for reprocessing and for refabrication. 


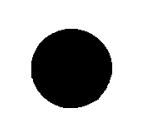

$$
\text { r }
$$
$\checkmark$

$\bullet$ 
CONTENTS

ABSTRACT . . . . . . . . . . . . . . . . . . . . iii

1. INTRODUCTION . . . . . . . . . . . . . . . . . . 1-1

2. HTGR FUEL CYCLE . . . . . . . . . . . . . . . . . 2-1

3. BLOCK FLOW FOR HTGR MARKET . . . . . . . . . . . . . . 3-1

4. FUEL ELEMENT COMPOSITION . . . . . . . . . . . . . . 4 4-1

4.1. Discussion . . . . . . . . . . . . . . . . 4-1

4.2. Method of Calculation ............... 4-2

4.2.1. Basic Calculationa1 Model . . . . . . . . 4 4-2

4.2.2. Reactions and Production Modes ......... . 4-3

4.2.3. Basic Data.............. 4-4

4.3. Impurities . . . . . . . . . . . . . . 4-4

4.4. Results .................. . . 4-19

5. CONCLUSIONS . . . . . . . . . . . . . . . . . 5-1

6. REFERENCES . . . . . . . . . . . . . . . . . . 6-1

\section{FIGURES}

1. HTGR recycle fuel schematic: once-through U-235 recycle, continuous U-233 recycle............... 2-2

2. I, M, 25R fissile particle chain............. 4-5

3. 23R fissile particle chain . . . . . . . . . . . . . 4-7

4. I, M, 23R, 25R fertile particle chain . . . . . . . . . . 4-9

5. Fission product and impurity chains . . . . . . . . . . 4-11

\section{TABLES}

1. HTGR fuel element block flow for recycle . . . . . . . . 3-3

2. Assumed concentration of impurities in graphite, thorium, and uranium in initial reactor fuel loadings . . . . . . . . 4-17 


\section{TABLES (Continued)}

3. Measured impurities for candidate HTGR graphites . . . . . . . 4-18

4. Reload 13 - fuel element composition as charged . . . . . . . 4-21

5. Reload 13 - fuel element composition as discharged . . . . . . 4-50 


\section{INTRODUCTION}

The High-Temperature Gas-Cooled Reactor (HTGR) utilizes the thoriumuranium fuel cycle. The initial HTGR core loading uses uranium enriched to $93 \%$ U-235 as feed fissile material with thorium fertile material for production of bred U-233. A 4-yr fuel cycle is employed with about onefourth of the core discharged and reloaded on an annual basis.

The bred U-233 and unburned U-235 are recovered, purified, and recycled as refabricated fuel elements. A typical HTGR progresses in its fuel cycle from the initial core loading to a quasi-equilibrium recycle core loading of three types of fuel elements containing the bred U-233, unburned U-235, and fresh U-235 makeup feed.

Using an HTGR market forecast, fuel element block flow data have been compiled. The compilation follows the start-up of the first reactors, the progression from the initial to the recycle core loadings, and the start-up and progression of the later reactors that make up the total market. The block flow data show the annual number of fuel elements discharged and the number avallable for reprocessing and refabrication. The compilation is extended to show the number of each type of fuel element involved in the fuel cycle.

Fuel element compositions, including the heavy metal, impurity, fission product, and activation product nuclides, were calculated for each type of fuel element included in a typical HTGR lifetime fuel cycle. The calculations included the initial, makeup, and recycle fuel elements at the time of reactor loading and when discharged after irradiation in the reactor core. For each type of fuel element, the nuclide compositions are compiled separately for the fissile and fertile material. 
In Volume I of this report, fuel element compositions are printed for a typical HTGR operating at equilibrium recycle with makeup fresh fuel elements, bred U-233 recycle fuel elements, and unburned U-235 recycle fuel elements. Volume II consists of an appendix containing the calculated fuel element compositions printed for selected cycles of a typical HTGR as the fuel cycle progresses from the inftial core loading to the equilibrium recycle core loading. In view of the publication costs and specialized interest, Volume II has been issued with a limited distribution. Requests for copies of Volume II should be directed to the Document Center, General Atomic Company. Archive computer tapes have been compiled and contain the fuel element composition data for each cycle over the life of a typical HTGR. Data from the tapes may be obtained by request to V. H. Pierce, General Atomic Company.

The fuel element composition and block flow data are the basis for establishing material and nuclide mass flow data for HTGR fuel reprocessing and refabrication. These data, in turn, are the basis for process stream material balance; by-product, effluent, and waste stream composition; and decay heat load, shielding, and criticality analyses. 


\section{INTRODUCTION}

The High-Temperature Gas-Cooled Reactor (HTGR) utilizes the thoriumuranium fuel cycle. The initial HTGR core loading uses uranium enriched to $93 \% \mathrm{U}-235$ as feed fissile material with thorium fertile material for production of bred U-233. A 4-yr fuel cycle is employed with about onefourth of the core discharged and reloaded on an annual basis.

The bred U-233 and unburned U-235 are recovered, purified, and recycled as refabricated fuel elements. A typica1 HTGR progresses in its fuel cycle from the initial core loading to a quasi-equilibrium recycle core loading of three types of fuel elements containing the bred U-233, unburned U-235, and fresh U-235 makeup feed.

Using an HTGR market forecast, fuel element block flow data have been compiled. The compilation follows the start-up of the first reactors, the progression from the initial to the recycle core loadings, and the start-up and progression of the later reactors that make up the total market. The block flow data show the annual number of fuel elements discharged and the number available for reprocessing and refabrication. The compilation is extended to show the number of each type of fuel element involved in the fuel cycle.

Fuel element compositions, including the heavy metal, impurity, fission product, and activation product nuclides, were calculated for each type of fuel element included in a typical HTGR lifetime fuel cycle. The calculations included the initial, makeup, and recycle fuel elements at the time of reactor loading and when discharged after irradiation in the reactor core. For each type of fuel element, the nuclide compositions are compiled separately for the fissile and fertile material. 
In Volume I of this report, fuel element compositions are printed for a typical HTGR operating at equilibrium recycle with makeup fresh fuel elements, bred U-233 recycle fuel elements, and unburned U-235 recycle fuel elements. Volume II consists of an appendix containing the calculated fuel element compositions printed for selected cycles of a typical HTGR as the fuel cycle progresses from the initial core loading to the equilibrium recycle core loading. In view of the publication costs and specialized interest, Volume II has been issued with a limited distribution. Requests for copies of Volume II should be directed to the Document Center, General Atomic Company. Archive computer tapes have been compiled and contain the fuel element composition data for each cycle over the life of a typical HTGR. Data from the tapes may be obtained by request to V. H. Pierce, General Atomic Company.

The fuel element composition and block flow data are the basis for establishing material and nuclide mass flow data for HTGR fuel reprocessing and refabrication. These data, in turn, are the basis for process stream material balance; by-product, effluent, and waste stream composition; and decay heat load, shielding, and criticality analyses. 


\section{HTGR FUEL CYCLE}

A schematic diagram of the HTGR thorium-uranium fuel cycle (Ref. 1) is shown in Fig. 1. Fissile material consisting of 93\% U-235 enriched uranium fabricated as fuel particles is mixed with thorium fertile fuel particles and fabricated into fuel elements, designated I type elements, for the initial reactor core loading.

To establish the 4-yr HTGR fuel loading cycle, about one-fourth of the I fuel elements are discharged following the first equivalent full-power year of reactor operation. A reload of the same number of elements is then made, and reactor operation is resumed.

The reload fuel elements are fabricated using U-235 fissile particles and thorium fertile particles but typically have greater fissile and fertile metal loadings per element than those used for the reactor initial core. The reload fuel elements containing 93\% U-235 enriched fissile material are designated makeup, $M$ type, fuel elements and have a 4-yr life in the reactor core.

The discharged, irradiated or spent, initial core fuel elements, designated IS elements, are shipped to storage facilities. The Is elements are subsequently reprocessed to recover the bred U-233 and unburned U-235 fissile material.

Annually, the remaining I fuel elements are discharged, at a rate of about one-fourth of the original number of elements each year. M elements are used for core annual reloading until recycle fuel elements become available. Discharged IS and subsequently spent makeup MS fuel elements are shipped to storage facilities. 


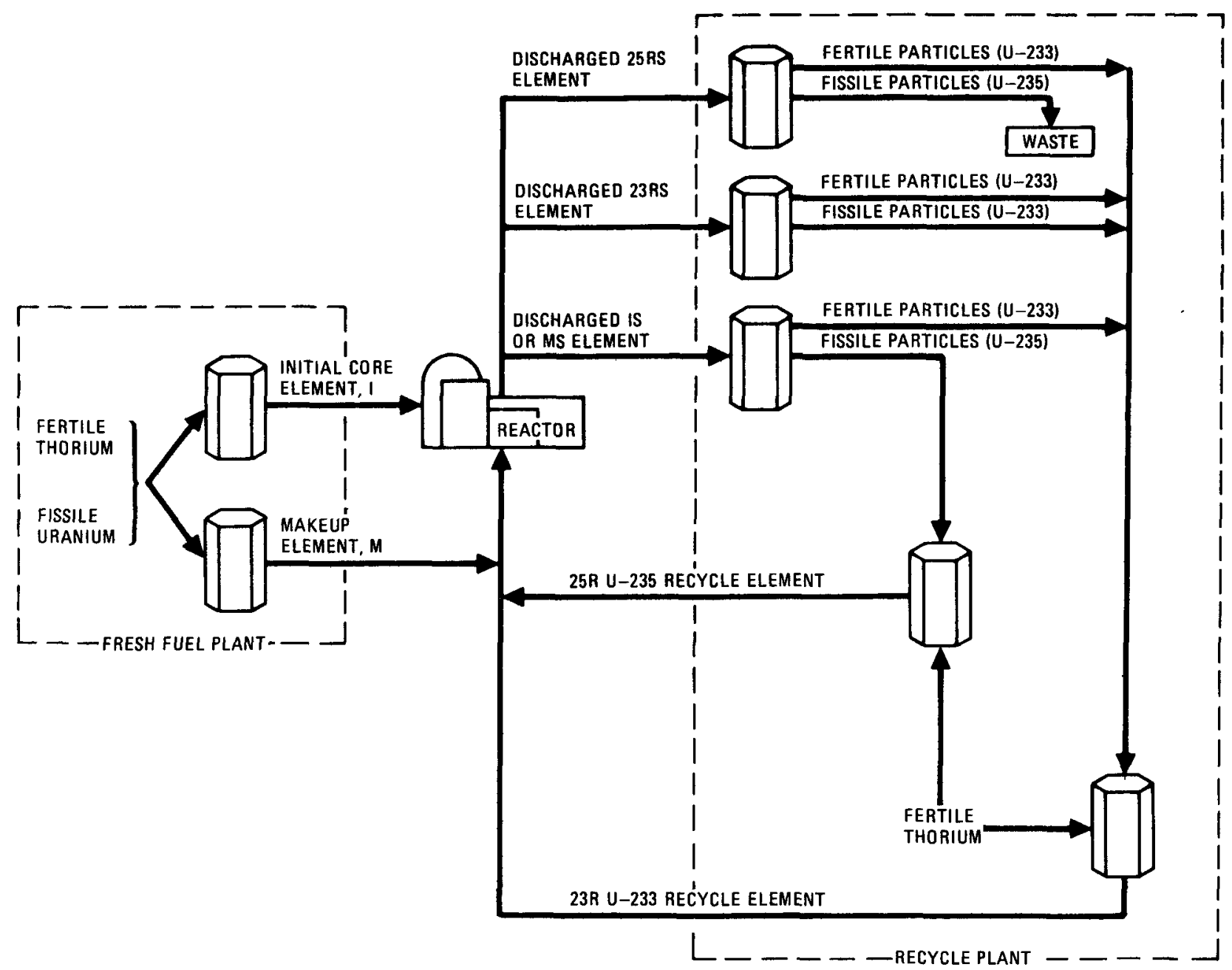

Fig. 1. HTGR recycle fuel schematic: once-through U-235 recycle, continuous $\mathrm{U}-233$ recycle 
The stored IS and MS elements are reprocessed to recover the U-233 fissile material bred in the fertile particles and the unburned U-235 fissile material (Refs, 2,3). The recovered fissile materials are refabricated into recycle fuel elements. Recycle elements made from the recovered U-235 material and the recovered U-233 material are designated $25 \mathrm{R}$ and $23 \mathrm{R}$ elements, respectively. Each type of recycle element is fabricated by mixing one kind of fissile particle with thorium fertile particles.

When the recycle elements become available, they are combined with $M$ elements for HTGR reloads. The total number of elements required for the annual reload is equal to the number of elements discharged at the given reload. The number of $23 \mathrm{R}$ and $25 \mathrm{R}$ elements comprising the total reload is determined by the number of these elements that can be refabricated from the available U-233 and U-235 fissile material. The number of $M$ elements is determined by the difference between the total number of fuel elements required and the number of recycle fuel elements that are available.

Typically, once recycle fuel is available, all fuel reloads for an HTGR are made up from M, 23R, and 25R fuel elements. Four years after introduction of recycle fuel, the reactor discharge will consist of the burned or spent MS, 23RS, and 25RS elements. After discharge these elements are shipped and reprocessed in a manner similar to that used for the IS and MS elements.

The MS elements are reprocessed to recover the bred U-233 and the unburned U-235. Unburned U-233 and bred U-233 are recovered from the 23RS elements. The twice-burned fissile particles containing U-235 are separated from the 25RS elements and retired as a high-level solid waste. The U-233 bred in the 25RS fertile particles is recovered for recycle. The two product streams--the U-235 fissile material from the MS elements and the U-233 fissile material combined from the MS, 23RS, and 25RS elements--are used to refabricate $25 R$ and $23 R$ recycle fuel elements. 


\section{BLOCK FLOW FOR HTGR MARKET}

The chronology of a typical HTGR fuel cycle development is important in the design of spent fuel storage, recycle plant, and fresh fuel plant facilities. In the initial stage of reactor operation, all discharged fuel is of the IS and MS types containing burned U-235 fissile material and bred U-233 material. When recycle $23 \mathrm{R}$ and $25 \mathrm{R}$ elements are introduced into the reactor fuel cycle, initially $\mathrm{few}$, if any, of the $\mathrm{M}$ elements are required. As the equilibrium fuel cycle is approached, a mix of $M, 23 R$, and $25 R$ elements is present, consisting of about $50 \% \mathrm{M}, 40 \% 23 \mathrm{R}$, and $10 \% 25 \mathrm{R}$ elements.

In order to minimize the contamination of the U-233 material with U-235 and the subsequent neutronic penalties due to U-236 buildup, separability of the U-235 material and the U-233-containing material is maintained (Ref. 4). A mechanical separation is provided by taking advantage of the properties of the different fuel particle coatings: silicon carbide TRISO coating on the fissile particles and pyrolytic carbon BISO coating on the fertile particles. Separate solvent extraction and purification systems--PUREX for the U-235 material and THOREX for the thorium/bred U-233 material--are provided. Separate refabrication processing of the $\mathrm{U}-235$ and $\mathrm{U}-233$ material into the $25 \mathrm{R}$ and $23 \mathrm{R}$ fuel elements is necessary.

To provide separate processing and accommodate the changes in the number and types of fuel elements requires sizing the recycle plant process systems to match the upper limits of the respective cases. To complete the forecast of the upper limit cases for the recycle fuel plant, a schedule mode1 for HTGR start-ups is required. Superimposing a single HTGR fue1 cycle history with a reactor start-up (and fuel element discharge) schedule will develop a recycle fuel plant forecast for required throughput.

In January 1976, the U.S. Energy Research and Development Administration (ERDA), Division of Nuclear Fuel Cycle and Production, forecast a schedule 
for HTGR spent fuel discharge for initial planning purposes. In its entirety the ERDA planning basis encompasses a number of qualifications including availability of recycle facilities, strategy for implementation of reactor fuel recycle, schedule for expansion of the recycle facility, and certain overall program planning elements. Although each of the qualifications in itself will represent a substantial planning effort, use of the basic HTGR fuel discharge schedule as forecast is illustrative of the potential recycle throughput variation as a function of the type of spent fuel element.

Table 1 shows the reactor discharge derived from the ERDA planning basis but extended to include IS, MS, 23RS, and 25RS fuel elements. No restrictions regarding the availability of recycle fuel facilities or programmatic strategies were used. The data represent a best case situation for fuel recycle in its simplest form.

Table 1 illustrates the changes that occur in reprocessing and refabrication requirements for a typical reactor market. The number of different types of fuel elements will change depending on the size of the market. However, the relation of the different types of elements to each other will be approximately as shown in the table.

In the early years of operation, the reprocessing head-end will be involved exclusively with IS and MS elements. The 23RS and 25RS elements do not enter head-end reprocessing for the first $5 \mathrm{yr}$. As shown in Table 1, the relation between the U-235 PUREX solvent extraction system and the thorium/U-233 THOREX system changes considerably. This is also true of the $23 \mathrm{R}$ and $25 \mathrm{R}$ refabrication systems. In both cases, in the first year of plant operation throughput will be approximately equal. The relationship will begin to change after the first year and will approach a ratio of 6 times as much U-233 throughput as U-235 throughput. Recycle plant and process system designs will be required to have sufficient flexibility to accommodate processing changes of the magnitude illustrated in Table 1. 
TABLE 1

HTGR FUEL ELEMENT BLOCK FLOW FOR RECYCLE $(a)$

\begin{tabular}{|c|c|c|c|c|c|c|c|c|c|}
\hline \multirow[b]{2}{*}{ Year } & \multicolumn{2}{|c|}{ LHTGRs Discharging } & \multicolumn{4}{|c|}{$\begin{array}{l}\text { Fuel Elements Available } \\
\text { for Reprocessing }\end{array}$} & \multicolumn{3}{|c|}{$\begin{array}{l}\text { Fuel Elements Available } \\
\text { for Refabrication }\end{array}$} \\
\hline & $1160 \mathrm{MW}(\mathrm{e})$ & $1550 \mathrm{MW}(\mathrm{e})$ & IS/MS & $23 R S$ & 25RS & Total & $23 \mathrm{R}$ & $25 R$ & Total \\
\hline 1989 & 1 & & 1,064 & & & 1,064 & 309 & 255 & 564 \\
\hline 1990 & 1 & & 960 & & & 960 & 346 & 86 & 432 \\
\hline 1991 & 1 & 1 & 2,336 & & & 2,336 & 793 & 359 & 1,152 \\
\hline 1992 & 1 & 3 & 5,088 & & & 5,088 & 1,706 & 813 & 2,519 \\
\hline 1993 & 1 & 5 & 7,944 & & & 7,944 & 2,831 & 981 & 3,812 \\
\hline 1994 & 1 & 7 & 9,908 & 309 & 255 & 10,472 & 3,888 & 1,057 & 4,945 \\
\hline 1995 & 1 & 9 & 12,672 & 346 & 86 & 13,104 & 5,047 & 1,136 & 6,183 \\
\hline 1996 & 1 & 12 & 16,080 & 793 & 359 & 17,232 & 6,684 & 1,549 & 8,233 \\
\hline 1997 & 1 & 16 & 20,321 & 1706 & 813 & 22,840 & 8,864 & 2,088 & 10,952 \\
\hline 1998 & 1 & 20 & 24,308 & 2831 & 981 & 28,120 & 11,060 & 2,330 & 13,390 \\
\hline 1999 & 1 & 24 & 28,439 & 3888 & 1057 & 33,384 & 13,348 & 2,485 & 15,833 \\
\hline 2000 & 1 & 28 & 32,705 & 5047 & 1136 & 38,888 & 15,747 & 2,647 & 18,394 \\
\hline
\end{tabular}

(a) Based on the HTGR discharged fuel element forecast for planning purposes made by ERDA, Division of Nuclear Fuel Cycle and Production, in January 1976. 


\section{FUEL ELEMENT COMPOSITION}

\subsection{DISCUSSION}

As a part of the development program for HTGR fuel recycle demonstration, data defining the composition of discharged spent fuel elements and fabricated recycle fuel elements are required. As described in the Introduction, the fuel cycle for an HTGR progresses through several stages in building to the quasi-equilibrium fuel loading. Included in the fuel cycle are the $I, M, 23 R$, and 25R type fuel elements (see Fig. 1).

The spent fuel elements discharged from a reactor contain a broad spectrum of heavy metal, impurity, fission product, and activation product nuclides. In the HTGR fuel, the particle form of the fertile and fissile materials results in a segregation of the irradiation products. The different fission yields and neutronic properties of the fertile and fissile materials and the segregation afforded by the particle form of the materials result in different irradiation product concentrations.

In the spent fuel reprocessing and fuel refabrication operations, the several fuel element components are segregated and handled in separate streams. Within the process streams the various unit operations result in by-products, waste, or products that are further segregated. The composition of the fuel element components for the various types of fuel elements provides the base line data for design purposes. The nuclides, nuclide masses, and nuclide activities are the basis for analyses of process flow streams, product composition, by-product and waste streams, decay heat loads, shielding, and criticality. 
4.2. METHOD OF CALCULATION

\subsubsection{Basic Calculationa1 Mode1}

The GARGOYLE code (Refs. 5,6) was used as the basic analytical model of the burnup occurring in the reactor core. Existing codes such as the ORIGEN code (Ref. 7), which is basically a one energy group code since the fast and resonance fluxes are held at a fixed ratio to the thermal flux, are not adequate to accurately model the buildup of long chains of activation products over the large shift in spectrum which occurs in going through the different operating modes (initial core, no recycle, partial recycle, full recycle, equilibrium recycle) of a typical HTGR life. The GARGOYLE code was used to calculate the flux spectrum and perform the burnup and activation calculations in nine energy groups (five fast and four thermal). For these calculations the GARGOYLE code represented the core as a point by using the core average nuclide concentration to determine the core average neutron spectrum in each group with core leakage introduced as positive or negative contributions to the fission source in each group. If desired, the GARGOYLE code could be used to calculate the local burnup of a single block by using the correct leakages for that particular location in the core. The nine group cross sections were prepared using the fourth edition of the Evaluated Nuclear Data File, Version B (ENDF/B-IV) (Ref. 8) with the MICROX code (Ref. 9), which calculates the correct spectrum for collapsing the cross sections from the input nuclide concentrations and lattice geometric data.

The GARGOYLE code is normally used to determine the fuel mass flows required for various cores and fuel cycles. For the present study the basic code was used to generate the mass flows and resulting core average fluxes for a typical fuel cycle in which recycle begins in reload 6 . The calculated masses of the primary feed materials and the fluxes were then stored on magnetic tape. A modified version of GARGOYLE (Ref. 10) retrieved this information from the tape and analytically irradiated the core feed materials and initial impurities in the neutron spectrum determined for the 
typical cycle. This technique allows the concentration of fission product, impurity, and activation product nuclides which have negligible neutronic importance to be calculated in a consistent spectrum without re-calculation of the core fluxes for each time point in the run. The modifications to the basic code reduced the mechanical calculation effort by a factor of 3 to 4 . A detailed description of these calculation methods and procedures will be provided in a forthcoming report (Ref. 11).

The basic method and modifications described above for the HTGR calculations are equally applicable for LWRs and other reactor systems.

\subsubsection{Reactions and Production Modes}

In the present study, the concentration in spent fuel of approximately 370 nuclides was calculated. Owing to the differences in production modes and the large quantity of information required, four separate basic production chains were constructed.

The heavy metal or actinide production chain from $\mathrm{T} 1-205$ to $\mathrm{Cm}-245$ was considered as transmutation and decay daughters of the thorium and uranium used to fuel the reactor. Since the initial concentrations of the various feed materials are considerably different for the thorium, U-235, and U-233 particles, minor modifications in the production chain were required. These modifications assume that the most important production modes are considered in each particle. The chains are shown schematically for the U-235-containing fissile particles, the U-233-containing fissile particles, and the thorium-containing fertile particles in Figs. 2, 3, and 4, respectively.

The fission product (and impurity activation) chains are illustrated schematically in Fig. 5. These chains include the nuclides produced by direct fission yield, the irradiation and decay daughters of the directly yielded nuclides, and many of the impurities along with their daughters. 


\subsubsection{Basic Data}

Most of the basic information required for this study was obtained from the third and fourth editions of the ENDF/B-IV data file. Many of the cross sections and all of the fission yields employed were obtained from ENDF/B-IV. The half-lives and production modes were taken from the "Chart of the Nuclides" (Ref. 12) and the "Table of Isotopes" (Ref. 13). The MICROX program was used to generate the nine broad-group cross sections from the ENDF/B data.

\subsection{IMPURITIES}

The impurity materials and concentrations in the raw materials used for fuel manufacturing used in these calculations are shown in Table 2 . For other impurity concentration levels, multiplying ratios can be used for many of the materials. However, it would be necessary to recalculate the fuel element compositions for some of the impurity materials contained in the thorium, uranium, or other material where the impurity is involved in the irradiation chains.

For comparison with the impurity materials and concentrations assumed in these calculations, recent impurity data determined for candidate graphites (Ref. 14) are shown in Table 3.

No impurities resulting from the reprocessing or refabrication operations have been included in the present data. Perfect classification has been assumed, i.e., no crossover of the U-235 fissile stream into the U-233 fertile stream or vice versa. In addition, solvent extraction decontamination factors of infinity have been assumed. No addition of process chemicals or equipment corrosion products is included.

The calculation method (Ref. 7) has the capability for user specification of factors to model impurities, contamination, and crossover. In general, decontamination factors and equipment corrosion products have a negligible effect on the subsequent recycle fuel irradiation or on the 


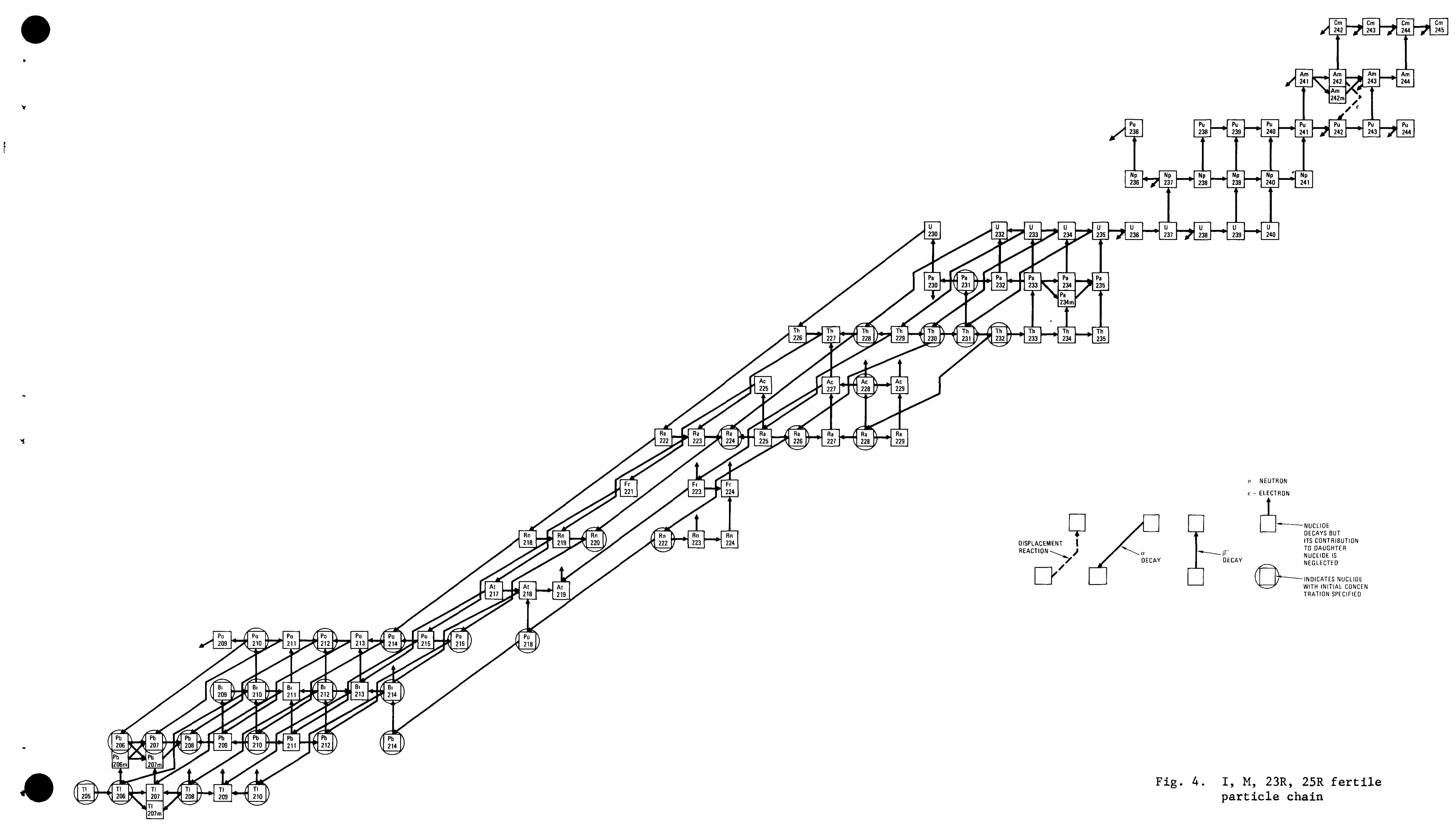




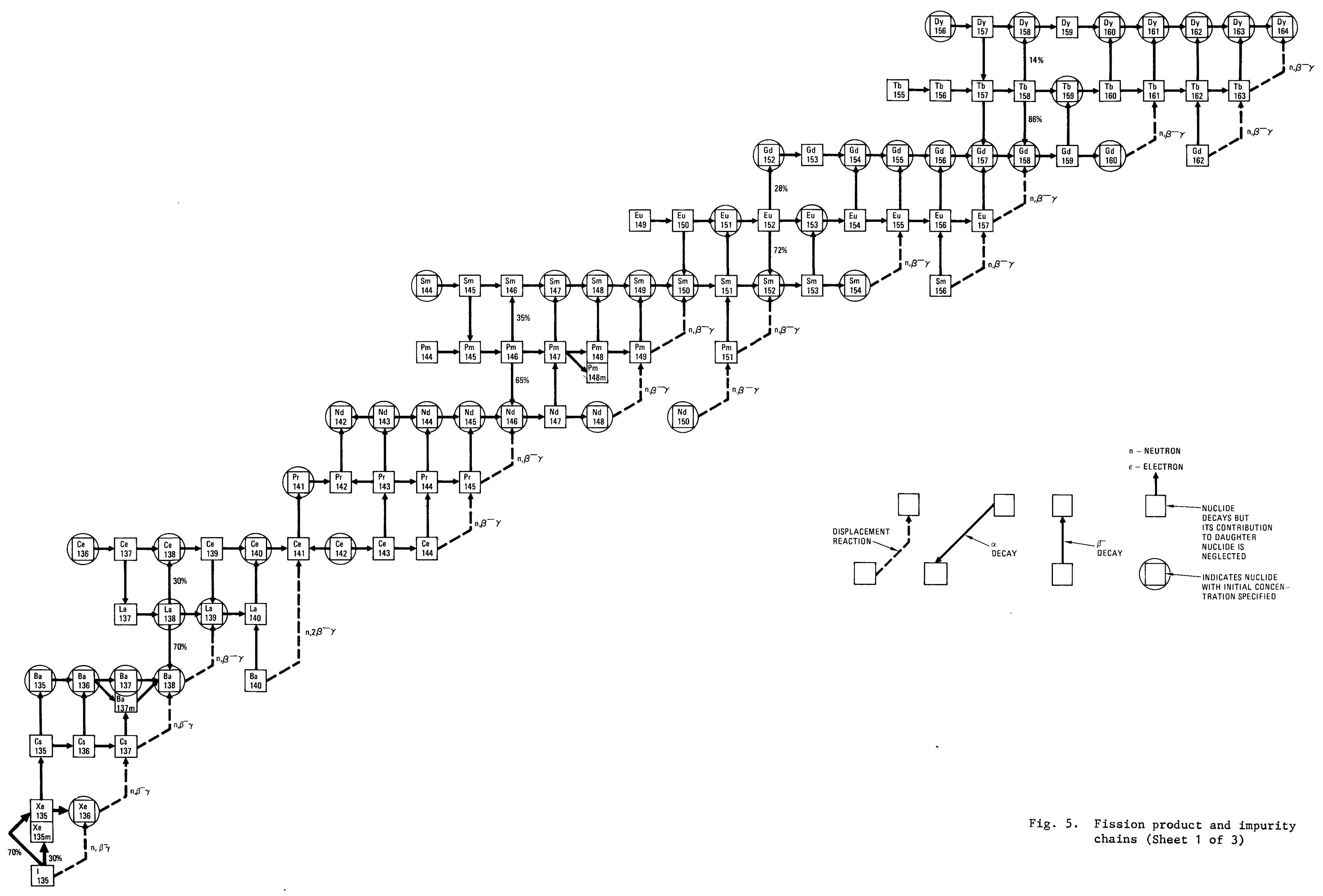




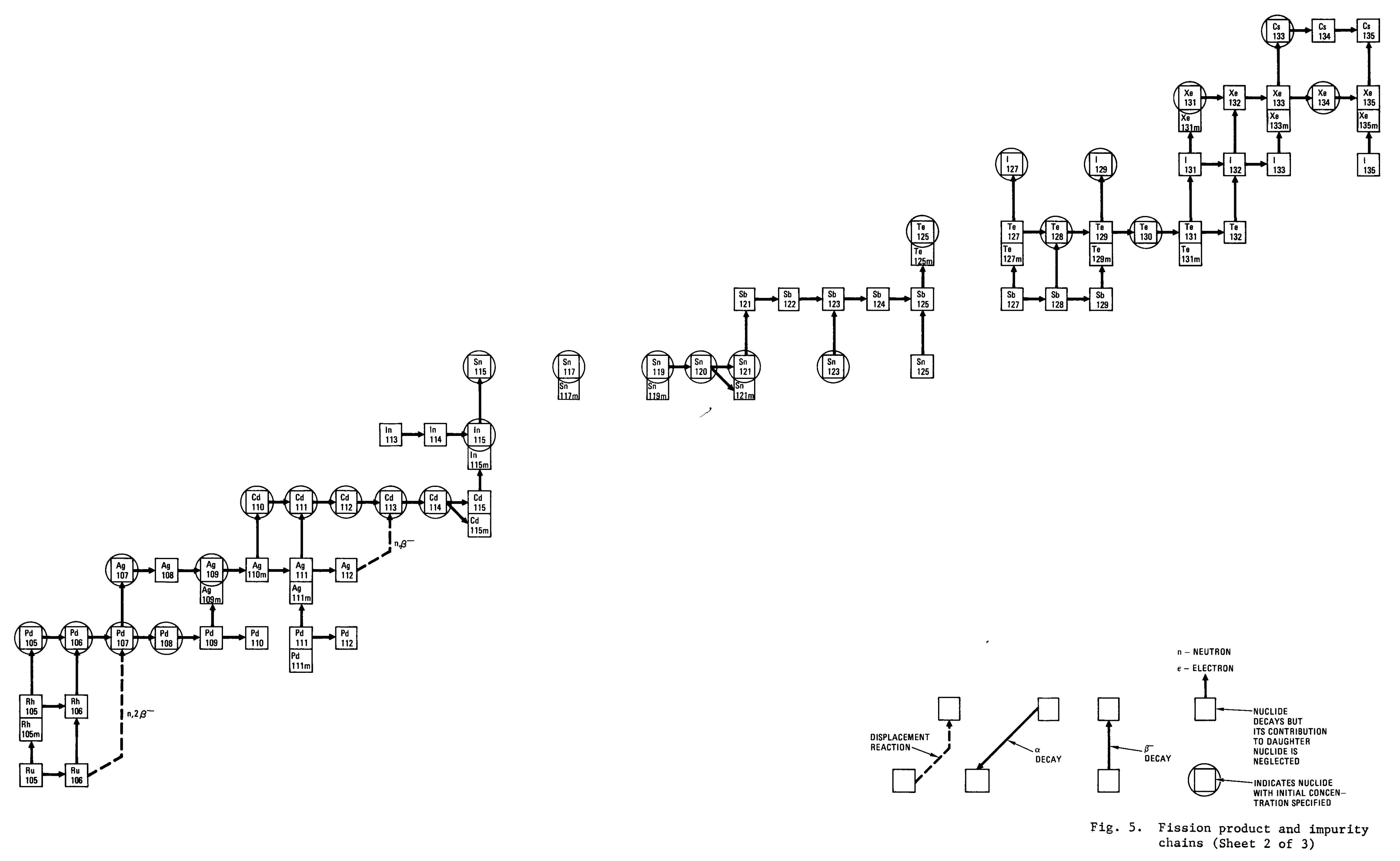




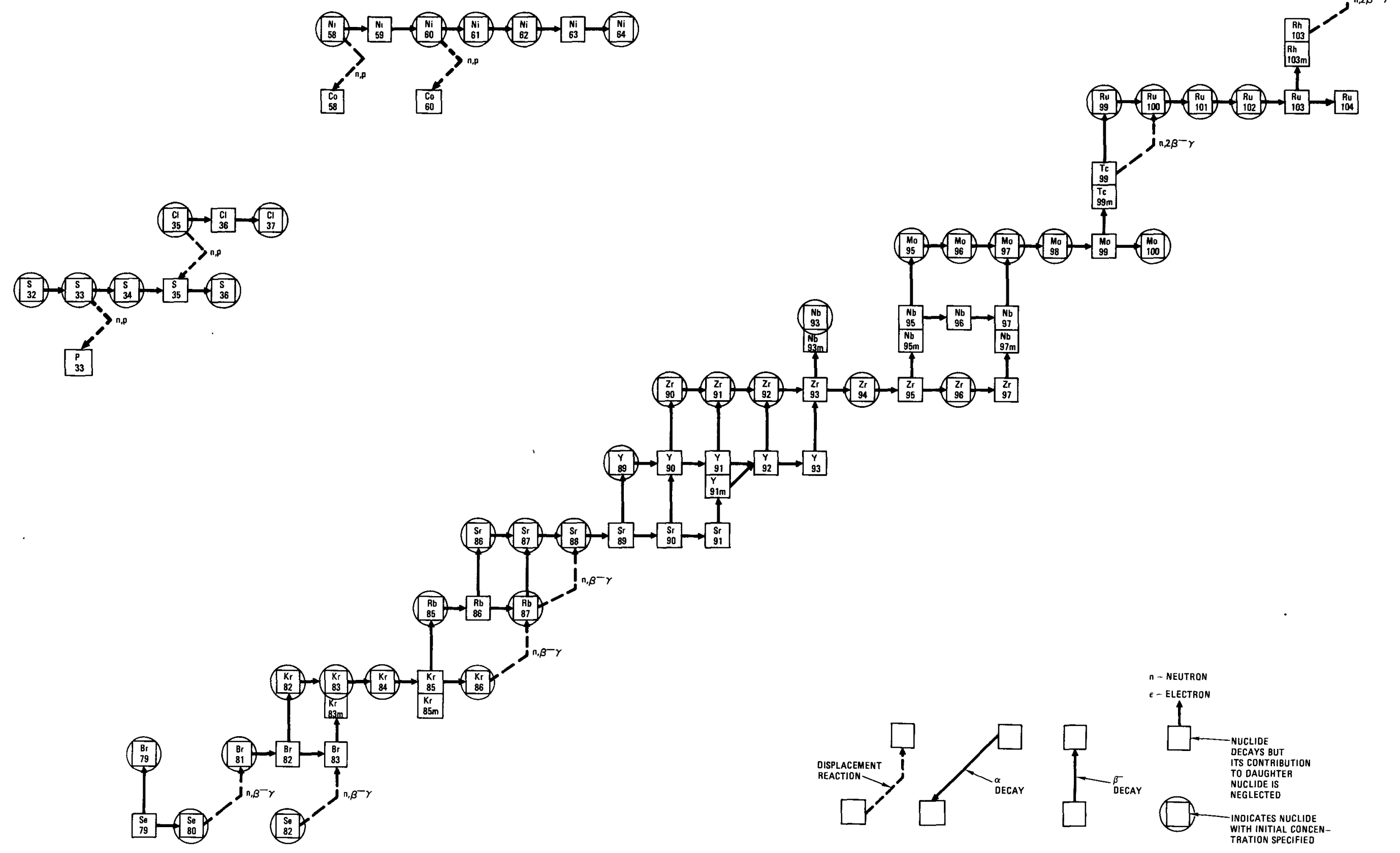


TABLE 2

ASSUMED CONCENTRATION OF IMPURITIES IN GRAPHITE, THORIUM, AND URANIUM IN INITIAL REACTOR FUEL LOADINGS

\begin{tabular}{|c|c|c|c|}
\hline \multirow[b]{2}{*}{ E1ement } & \multicolumn{3}{|c|}{ Concentration (ppm) } \\
\hline & Graphite & Thorium & Uranium \\
\hline $\mathrm{Ag}$ & 0 & 0.5 & 0.5 \\
\hline B & 59.5 & 0.5 & 0.5 \\
\hline $\mathrm{Ba}$ & 0 & 10 & 10 \\
\hline $\mathrm{Bi}$ & 0 & 2 & 2 \\
\hline $\mathrm{Cd}$ & 0.05 & 0.5 & 0.5 \\
\hline $\mathrm{Ce}$ & 0 & 50 & 50 \\
\hline C1 & 3 & 0 & 0 \\
\hline Co & 0 & 5 & 5 \\
\hline Cs & 0 & 10 & 10 \\
\hline Eu & 0.0001 & 0 & 0 \\
\hline Gd & 0.02 & 0 & 0 \\
\hline In & 0.4 & 5 & 5 \\
\hline La & 0 & 50 & 50 \\
\hline Li & 1 & 2 & 2 \\
\hline Mo & 0 & 50 & 50 \\
\hline $\mathrm{N}$ & 1 & 1 & 1 \\
\hline $\mathrm{Nb}$ & 0 & 50 & 50 \\
\hline $\mathrm{Ni}$ & 10 & 100 & 100 \\
\hline 0 & 1 & 1 & 1 \\
\hline $\mathrm{P}$ & 0 & 50 & 50 \\
\hline $\mathrm{Pb}$ & 0 & 5 & 5 \\
\hline $\mathbf{S}$ & 200 & 50 & 50 \\
\hline $\mathrm{Sm}$ & 0.01 & 0 & 0 \\
\hline $\mathrm{Sb}$ & 0 & 10 & 10 \\
\hline Si & 100 & 100 & 100 \\
\hline Sn & 0 & 10 & 10 \\
\hline $\mathrm{Sr}$ & 0 & 20 & 20 \\
\hline Th (a) & 0 & 0 & 15 \\
\hline U & 0 & (b) & 0 \\
\hline $\mathrm{Zr}$ & 0 & 50 & 50 \\
\hline
\end{tabular}

(a) Th-230 impurity in thorium is assumed to be $100 \mathrm{ppm}$. Resulting decay daughters from 2 yr of decay are also assumed.

(b) Resulting decay daughters from 2 yr of decay are also assumed. 
TABLE 3

MEASURED IMPURITIES FOR CANDIDATE HTGR GRAPHITES

\begin{tabular}{|c|c|c|c|}
\hline Element & $\begin{array}{l}\text { Impurity } \\
\text { Content } \\
\text { (ppm) }\end{array}$ & Element & $\begin{array}{l}\text { Impurity } \\
\text { Content } \\
\text { (ppm) }\end{array}$ \\
\hline \multicolumn{2}{|c|}{ Nonburnable } & $\mathrm{Ni}$ & $<4.0$ \\
\hline & & 0 & -- \\
\hline $\mathrm{Ag}$ & $<0.5$ & $\mathrm{P}$ & $<1.0$ \\
\hline $\mathrm{A} 1$ & $<3.7$ & $\mathrm{~Pb}$ & $<6.9$ \\
\hline $\mathrm{Ba}$ & $<0.5$ & $\operatorname{Pr}$ & $<0.42$ \\
\hline $\mathrm{Be}$ & $<0.005$ & $\mathrm{Rb}$ & $<1.0$ \\
\hline $\mathrm{Bi}$ & $<1.0$ & $S$ & $<1.6$ \\
\hline $\mathrm{Ca}$ & $<21.5$ & $\mathrm{Sb}$ & $<1.0$ \\
\hline $\mathrm{Ce}$ & $<0.30$ & $\mathrm{Sc}$ & $<0.01$ \\
\hline $\mathrm{Cl}$ & $<3.0$ & $\mathrm{Si}$ & $<26.0$ \\
\hline Co & $<4.0$ & $\mathrm{Sn}$ & $<1.0$ \\
\hline $\mathrm{Cr}$ & $<1.0$ & $\mathrm{Sr}$ & $<0.30$ \\
\hline $\mathrm{Cs}$ & $<1.0$ & $\mathrm{Ta}$ & $<0.35$ \\
\hline $\mathrm{Cu}$ & $<0.07$ & $\mathrm{~Tb}$ & $<0.20$ \\
\hline Dy & $<0.12$ & $\mathrm{~T} i$ & $<1.2$ \\
\hline $\operatorname{Er}$ & $<0.03$ & $\mathrm{Tl}$ & $<1.0$ \\
\hline $\mathrm{Fe}$ & $<3.0$ & $\mathrm{Tm}$ & $<0.04$ \\
\hline $\mathrm{Hf}$ & $<0.17$ & $\mathrm{~V}$ & $<0.5$ \\
\hline Ho & $<0.08$ & $\mathrm{~W}$ & $<25.5$ \\
\hline In & $<1.0$ & $\mathrm{Yb}$ & $<0.03$ \\
\hline $\mathrm{K}$ & $<1.0$ & $\mathrm{Zn}$ & $<1.0$ \\
\hline $\mathrm{La}$ & $<0.03$ & $\mathrm{Zr}$ & $<0.08$ \\
\hline $\mathrm{Lu}$ & $<0.02$ & & \\
\hline $\mathrm{Mg}$ & $<0.8$ & \multicolumn{2}{|c|}{ Burnable } \\
\hline $\mathrm{Mn}$ & $<1.0$ & & \\
\hline Mo & $<1.0$ & B & $<2.4$ \\
\hline $\mathrm{N}$ & - & $\mathrm{Cd}$ & $<0.5$ \\
\hline $\mathrm{Na}$ & $<10.3$ & $\mathrm{Eu}$ & $<0.04$ \\
\hline $\mathrm{Nb}$ & $<0.05$ & $\mathrm{Gd}$ & $<0.05$ \\
\hline \multirow[t]{2}{*}{ Nd } & $<0.3$ & $\mathrm{Li}$ & $<0.005$ \\
\hline & & $\mathrm{Sm}$ & $<0.39$ \\
\hline
\end{tabular}


irradiated recycle fuel composition. The production of the irradiation nuclides substantially exceeds nuclide concentration as an impurity. However, the presence of these impurities in the refabrication feed is important in terms of the feed specifications, process and support system design, shielding requirements, decay heat loads, and criticality. Future calculations can be made to estimate the effect on refabrication operations or at a time when process specifications are available establishing a range for impurity concentrations.

\subsection{RESULTS}

The calculational method described in Section 4.2 was used to determine the average spent fuel composition for a typical HTGR operating at equilibrium recycle with $M, 23 R$, and 25R type fuel elements, reload 13 . For the reload, two data tabulations are included: (1) Table 4, the composition of the elements as originally charged, and (2) Table 5, the composition and activity of the discharged fuel elements at 180 days after reactor shutdown. Volume II of this report ${ }^{*}$ contains data in a printed form for the following selected HTGR reloads:

Reload 1 - initial core IS type fuel elements discharged after one equivalent full-power year.

Reload 5 - MS type fuel elements (and IS type elements) discharged after $4 \mathrm{yr}$ of burnup. Representative of $M$ type fuel elements for non-recycle reloads.

Reload 10 - only 23RS and 25RS fuel elements (no M type elements used) discharged after $4 \mathrm{yr}$ of burnup.

Reload 13 - MS, 23RS, and 25RS elements discharged after 4 yr of burnup. Typical for an HTGR operating at equilibrium recycle.

${ }^{*}$ Requests for copies of Volume II should be directed to the Document Center, General Atomic Company. Printed tabulations for other HTGR reloads may be obtained by request to V. H. Pierce, General Atomic Company. 
For each discharge or reload, two data tabulations are included: the composition and activity of the reload fuel elements and of the discharged spent fuel elements. The spent fuel element tabulations are presented for 180-day, 1-yr, 3-yr, 5-yr, and 10-yr decay times after reactor shutdown.

In addition to the reloads printed in Volume II of this report, computer tape data files are in archives for all the other reactor reloads. The files can be used to calculate and print the composition of the fuel elements for any other reload. Also, the spent fuel element composition and activity data can be calculated and printed for any specified decay times beginning $24 \mathrm{hr}$ after reactor shutdown. 
TABLE 4

RELOAD 13 - FUEL ELEMENT COMPOSITION AS CHARGED

MS, 23RS, and 25RS elements are discharged at reload 13. The elements were originally loaded at reload 9. The $M$ elements contain fertile thorium particles and U-235 fully enriched uranium particles. The 23R and 25R elements were fabricated using bred U-233 material and unburned U-235 recovered and purified from reprocessing of earlier discharged MS, 23RS, and 25RS elements. 
NUCLIDE WEIGHTS IN GRAMS PER F.E. FOR RELOAD 9

\begin{tabular}{|c|c|c|c|c|c|c|}
\hline NUCLIDES & $\begin{array}{cl}\text { I,M } & \text { FRESH } \\
\text { FERTILE PTL. }\end{array}$ & $\begin{array}{l}U-235 F \cdot E \\
\text { FISSILE PTL. }\end{array}$ & $\begin{array}{l}23 R \text { RECYCLE } \\
\text { FERTILE PTL. }\end{array}$ & $\begin{array}{l}\text { U-233 F.E. } \\
\text { FISSILE PTL. }\end{array}$ & $\begin{array}{l}\text { 25R RECYCLE } \\
\text { FERTILE PTL. }\end{array}$ & $\begin{array}{l}\text { U-235 F.E. } \\
\text { FISSILE PIL. }\end{array}$ \\
\hline$--\infty-\infty-\infty$ & $-----------\infty$ & 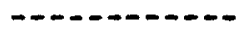 & 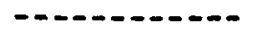 & 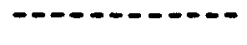 & 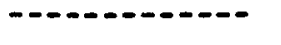 & 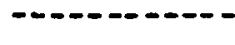 \\
\hline $\begin{array}{l}C M-242 \\
C M-243 \\
C M-244 \\
C M-245 \\
\text { CURIUM }\end{array}$ & $\begin{array}{l}0.0000 \\
0.0000 \\
0.0000 \\
0.0000 \\
c .0000\end{array}$ & $\begin{array}{l}0.0000 \\
0.0000 \\
0.0000 \\
0.0000 \\
0.0000\end{array}$ & $\begin{array}{l}0.0000 \\
0.0000 \\
0.0000 \\
0.0000 \\
0.0000\end{array}$ & $\begin{array}{l}0.0000 \\
0.0000 \\
0.0000 \\
0.0000 \\
0.0000\end{array}$ & $\begin{array}{l}0.0 \cap 00 \\
0 . \text { undo } \\
0 . \operatorname{crco} \\
0.0 ก 20 \\
0.0 \cap 00\end{array}$ & $\begin{array}{l}0.0000 \\
0.0700 \\
0.0 r 00 \\
0.0000 \\
0.0 r 00\end{array}$ \\
\hline $\begin{array}{l}A M-241 \\
A M-242 M \\
A M-242 \\
A M-243 \\
A M-244 \\
A M E R I C I U M\end{array}$ & $\begin{array}{l}0.0000 \\
0.0000 \\
0.0000 \\
0.0000 \\
0.0000 \\
0.0000\end{array}$ & $\begin{array}{l}0.0000 \\
0.0000 \\
0.0000 \\
0.0000 \\
0.5000 \\
0.0000\end{array}$ & $\begin{array}{l}0.0000 \\
c .0000 \\
0.0000 \\
0.0300 \\
c .0005 \\
0.0000\end{array}$ & $\begin{array}{l}0.0000 \\
0.0000 \\
0.0000 \\
0.0000 \\
0.0000 \\
0.0000\end{array}$ & $\begin{array}{l}0.0000 \\
0.0000 \\
0.0700 \\
0.0 \cap 00 \\
0.0 \cap 00 \\
0.0 \div 00\end{array}$ & $\begin{array}{l}0.0000 \\
0.0000 \\
0.0000 \\
0.0000 \\
0.0200 \\
0.0000\end{array}$ \\
\hline $\begin{array}{l}P U-236 \\
P U-238 \\
P U-239 \\
P U-24 C \\
P U-241 \\
P U-242 \\
P U-243 \\
P U-244 \\
P L U T O N I U M\end{array}$ & $\begin{array}{l}0.0000 \\
0.0000 \\
0.0000 \\
0.0000 \\
0.0000 \\
0.0000 \\
0.0000 \\
0.0000 \\
0.0000\end{array}$ & $\begin{array}{l}0.0000 \\
0.0000 \\
0.0000 \\
0.000 n \\
0.0000 \\
0.0000 \\
0.0000 \\
0.0000 \\
0.0000\end{array}$ & $\begin{array}{l}0.0000 \\
0.0000 \\
0.0000 \\
0.0000 \\
0.0020 \\
0.0000 \\
0.0000 \\
0.0007 \\
0.0000\end{array}$ & $\begin{array}{l}0.0000 \\
0.0000 \\
0.0000 \\
0.0000 \\
0.0 .000 \\
0.0000 \\
0.0000 \\
0.0000 \\
0.0000\end{array}$ & $\begin{array}{l}0.0000 \\
0.0000 \\
0.0000 \\
0.0000 \\
0.0 ? 100 \\
0.0000 \\
0.0 n 00 \\
0.0300 \\
0.0700\end{array}$ & $\begin{array}{l}0.0000 \\
0.0000 \\
0.0000 \\
0.0000 \\
0.0000 \\
0.0000 \\
0.0000 \\
0.0000 \\
0.0000\end{array}$ \\
\hline $\begin{array}{l}N P-236 \\
N P=237\end{array}$ & $\begin{array}{l}0.0000 \\
0.0 ? 00\end{array}$ & $\begin{array}{l}0.0000 \\
0.0000\end{array}$ & $\begin{array}{l}\text { C. ODCo } \\
0.3000\end{array}$ & $\begin{array}{l}0.0000 \\
0.0 r 00\end{array}$ & $\begin{array}{l}0.4000 \\
0.0005\end{array}$ & $\begin{array}{l}0.0000 \\
0.0000\end{array}$ \\
\hline $\begin{array}{l}\text { NP }-238 \\
\text { NP- } 239 \\
\text { NP-?4C } \\
\text { NP-241 } \\
\text { NEPTUNIUM }\end{array}$ & $\begin{array}{l}0.0000 \\
0.0000 \\
0.0000 \\
0.0000 \\
0.0000\end{array}$ & $\begin{array}{l}0.0000 \\
0.0000 \\
0.0000 \\
0.0000 \\
0.0000\end{array}$ & $\begin{array}{l}0.0000 \\
0.0000 \\
0.0000 \\
0.0000 \\
0.0000\end{array}$ & $\begin{array}{l}0.0000 \\
0.0000 \\
0.0000 \\
0.0000 \\
0.0000\end{array}$ & $\begin{array}{l}0.0300 \\
0.0000 \\
0.0000 \\
0.0 n 50 \\
0.0900\end{array}$ & $\begin{array}{l}0.0 c 00 \\
3.0703 \\
0.0000 \\
0.0000 \\
0.0 \cap 00\end{array}$ \\
\hline $\begin{array}{l}u-230 \\
u-232 \\
u-233 \\
u-234 \\
u-235\end{array}$ & $\begin{array}{l}0.0000 \\
0.0000 \\
0.0000 \\
0.0000 \\
0.0000\end{array}$ & $\begin{array}{l}0.0000 \\
0.0000 \\
0.0000 \\
6.1802+00 \\
7.7926+02\end{array}$ & $\begin{array}{l}0.0000 \\
0.0000 \\
0.0000 \\
0.000 n \\
0.0000\end{array}$ & $\begin{array}{l}0.0000 \\
3.0944-01 \\
5.4462+02 \\
1.1 \times 20+02 \\
2.3186+01\end{array}$ & $\begin{array}{l}0.0000 \\
0.0000 \\
0.0000 \\
0.0700 \\
0.0000\end{array}$ & $\begin{array}{l}0.0000 \\
0.0000 \\
0.0000 \\
2.5636+01 \\
5.767 .1+02\end{array}$ \\
\hline
\end{tabular}


NUCLIDE WEIGHTS IN GRAMS PER F.E. FOR RELOAD?

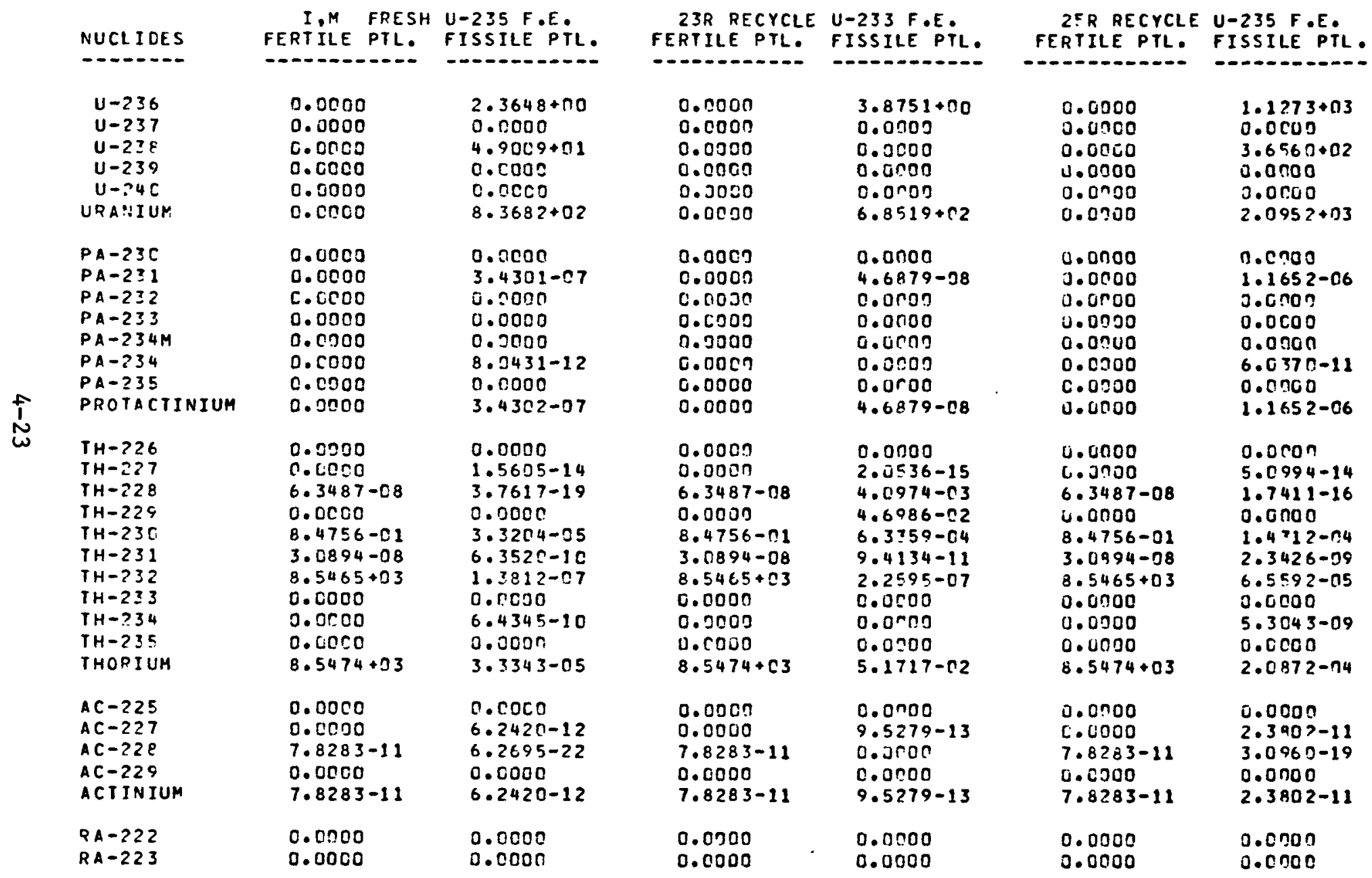


NUCLIDE WEIGHTS IN GRAMS PER F.E. FOR RELOAD?

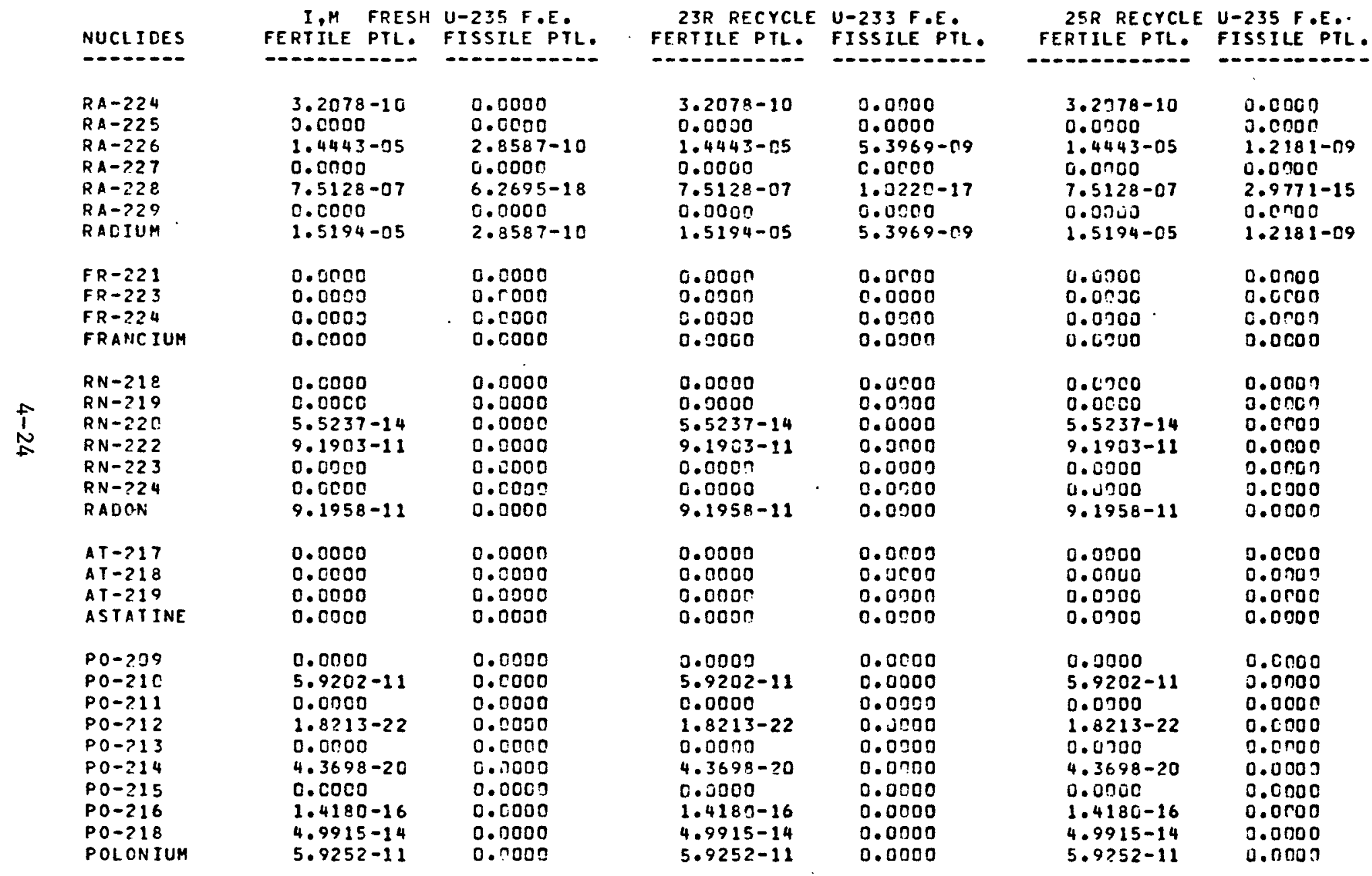


THESE ARE THE CONCENTRATIONS AS LOADED FOR RELOAD

NUCLIOE WEIGHTS IN GRAMS PER F.E. FOR RELOAD 9

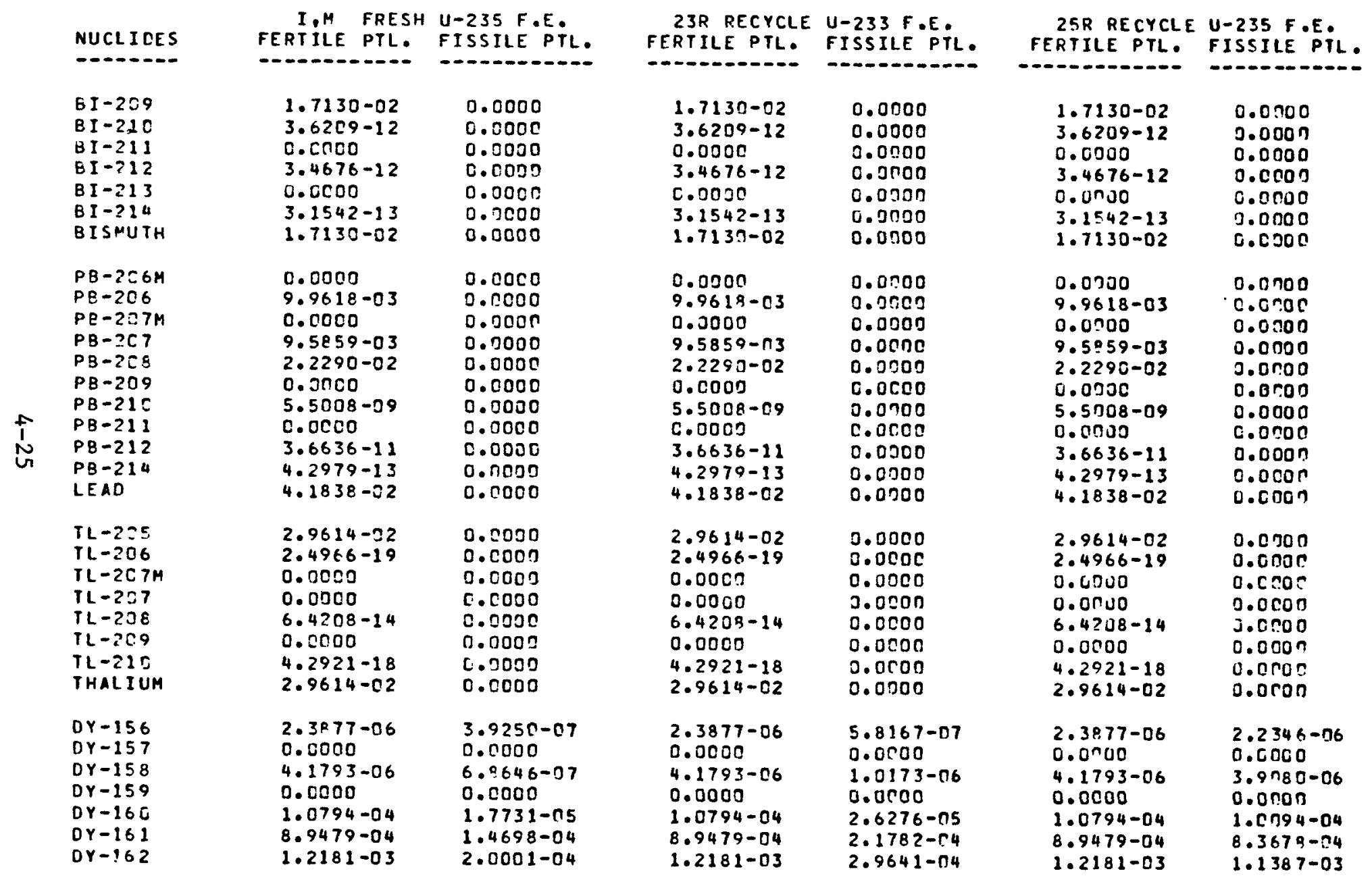


NUCLIOE WEIGHTS IN GRAMS PER F.E. FOR RELOAD 9

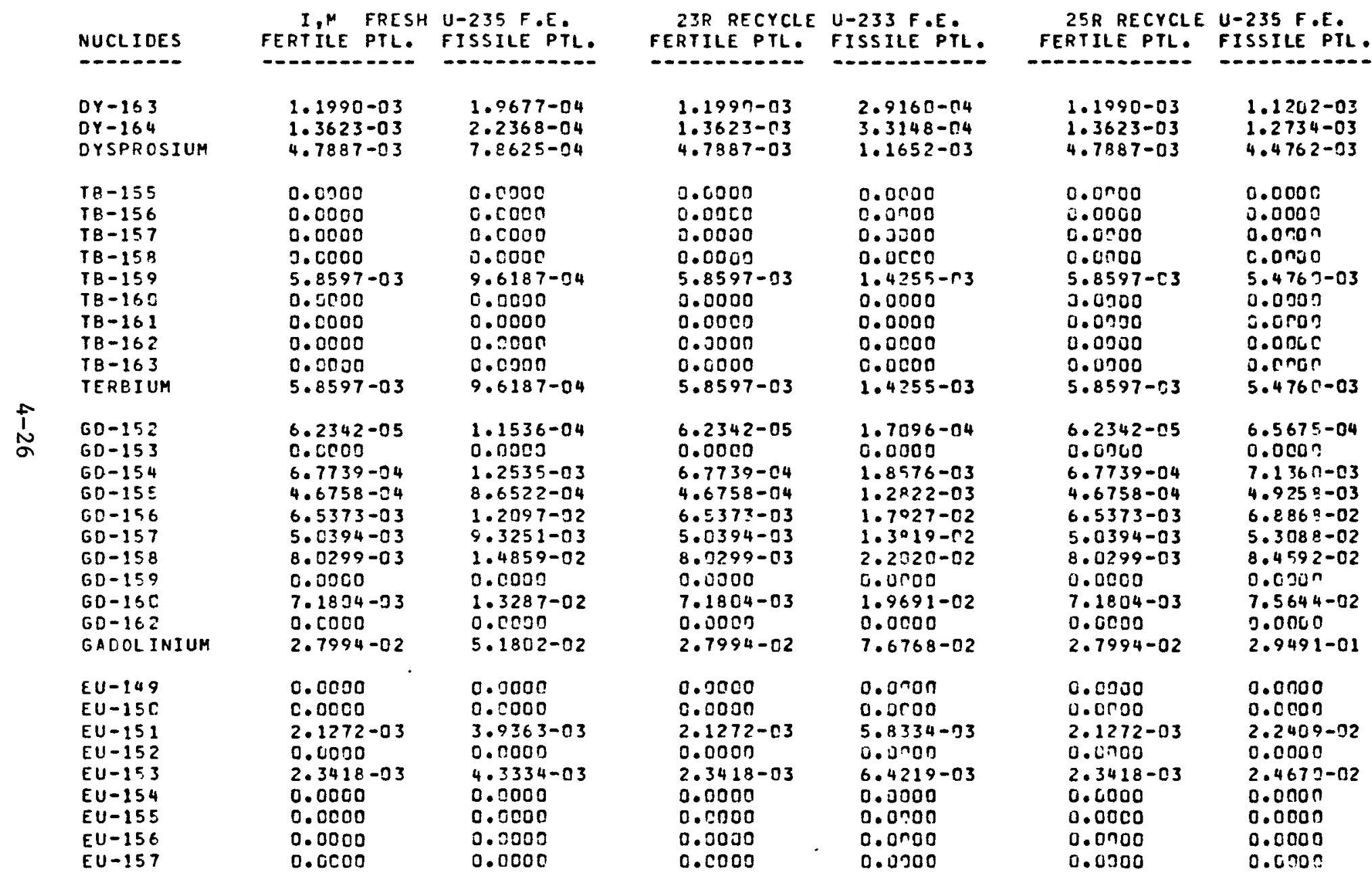


NUCL IDE WEIGHTS IN GRAMS PER F,E. FOR RELOAD?

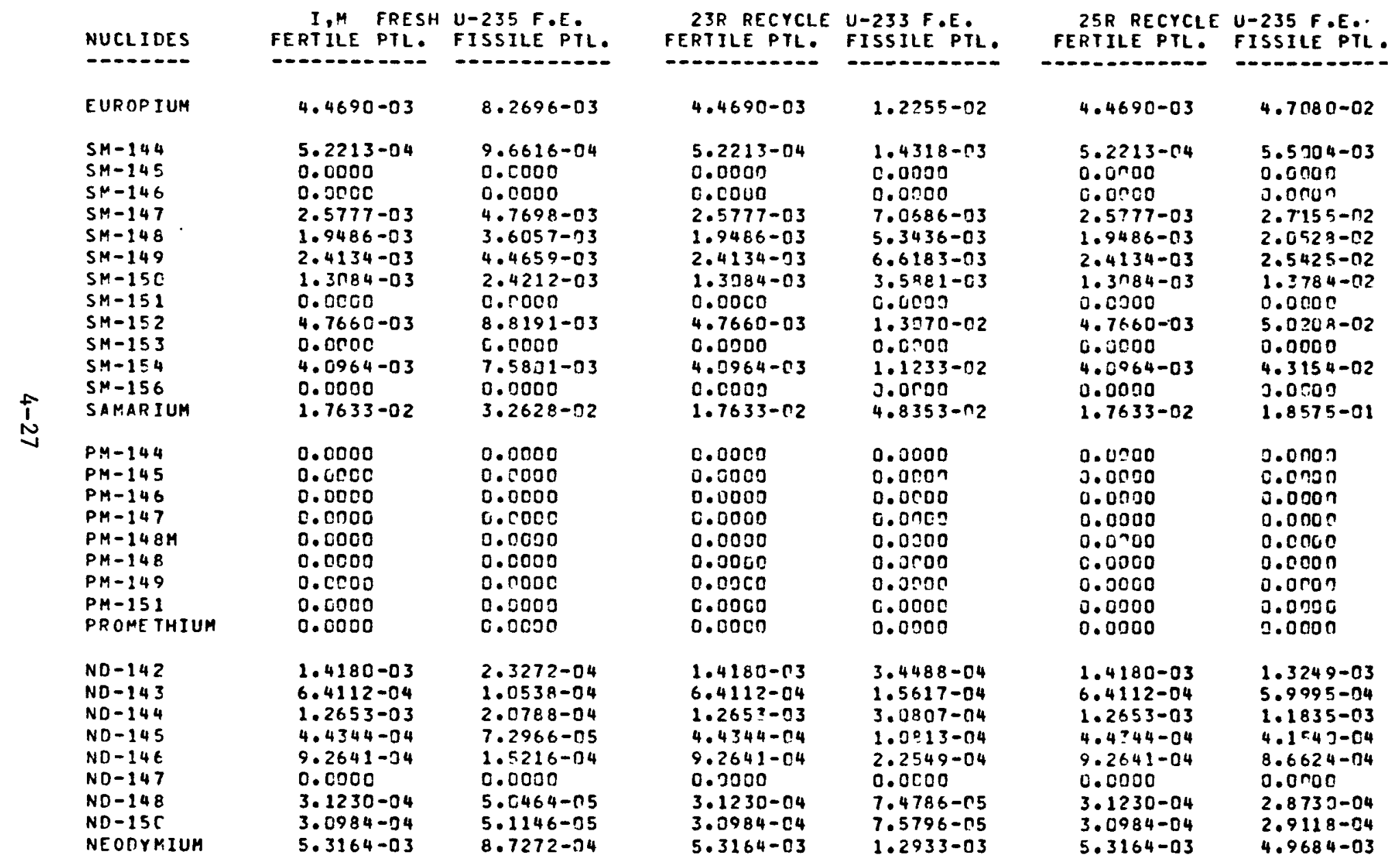


NUCLIDE HEIGHTS IN GRAMS PER F.E. FOR RELOAD 9

\begin{tabular}{|c|c|c|c|c|c|c|}
\hline NUCLIDES & $\begin{array}{cl}\text { I,M } & \text { FRESH } \\
\text { FERTILE PTL. }\end{array}$ & $\begin{array}{l}\text { U-235 F.E. } \\
\text { FISSILE PTL. }\end{array}$ & $\begin{array}{l}23 R \text { RECYCLE } \\
\text { FERTILE PTL. }\end{array}$ & $\begin{array}{l}\text { U-233 F.E. } \\
\text { FISSILE PTL. }\end{array}$ & $\begin{array}{l}25 R \text { RECYCLE } \\
\text { FERTILE PTL. }\end{array}$ & $\begin{array}{l}\text { U-235 F .E. } \\
\text { FISSILE PTL. }\end{array}$ \\
\hline$\ldots$ & 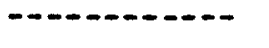 & 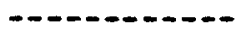 & $-2-2-2-20-2$ & 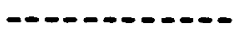 & $-2-20-2-2-2$ & $----2--2-2$ \\
\hline $\begin{array}{l}P R-141 \\
P R-142 \\
P R-143 \\
P R-144\end{array}$ & $\begin{array}{l}5.1942-03 \\
0.0000 \\
0.0000 \\
0.0000\end{array}$ & $\begin{array}{l}8.5298-04 \\
0.0000 \\
0.0000 \\
0.5000\end{array}$ & $\begin{array}{l}5.1942-03 \\
0.0000 \\
0.0000 \\
0.0000\end{array}$ & $\begin{array}{l}1.2641-03 \\
0.0000 \\
0.0000 \\
0.0000\end{array}$ & $\begin{array}{l}5.1942-03 \\
0.0000 \\
3.0000 \\
0.0000\end{array}$ & $\begin{array}{l}4.8561-03 \\
0.0000 \\
0.0009 \\
0.0000\end{array}$ \\
\hline $\begin{array}{l}\text { PR- } 145 \\
\text { PRASE CDYMIUM }\end{array}$ & $\begin{array}{l}0.0000 \\
5.1942-03\end{array}$ & $\begin{array}{l}0.0002 \\
8.5298-04\end{array}$ & $\begin{array}{l}0.0000 \\
5.1942-.03\end{array}$ & $\begin{array}{l}0.0000 \\
1.2641-03\end{array}$ & $\begin{array}{l}0.0300 \\
5.1942-03\end{array}$ & $\begin{array}{l}0.0000 \\
4.8561-03\end{array}$ \\
\hline $\begin{array}{l}C E-136 \\
C E-137 \\
C E-138 \\
C E-139 \\
C E-140 \\
C E-141 \\
C E-142 \\
C E-143 \\
C E-144 \\
C E F I U M\end{array}$ & $\begin{array}{l}4.8342-04 \\
0.0000 \\
6.3551-04 \\
0.0000 \\
2.2822-01 \\
0.0000 \\
2.8951-02 \\
0.0000 \\
0.0000 \\
2.5829-01\end{array}$ & $\begin{array}{l}7.9282-05 \\
0.0000 \\
1.0435-04 \\
0.0000 \\
3.7458-02 \\
0.0000 \\
4.7637-03 \\
0.0000 \\
0.0000 \\
4.2405-02\end{array}$ & $\begin{array}{l}4.8342-04 \\
0.0000 \\
6.3551-04 \\
0.0000 \\
2.2822-01 \\
0.0000 \\
2.8951-02 \\
0.0000 \\
0.0000 \\
2.5829-01\end{array}$ & $\begin{array}{l}1.1749-54 \\
0.0700 \\
1.5465-04 \\
0.0000 \\
5.5511-02 \\
0.0200 \\
7.0596-03 \\
0.0000 \\
0.0000 \\
6.2042-02\end{array}$ & $\begin{array}{l}4.8342-04 \\
0.0000 \\
6.3551-04 \\
0.6000 \\
2.2822-01 \\
0.0000 \\
2.8951-02 \\
0.0000 \\
0.0000 \\
2.5829-01\end{array}$ & $\begin{array}{l}4.5136-04 \\
0.0000 \\
5.9410-04 \\
0.0000 \\
2.1325-01 \\
0.0000 \\
2.7120-02 \\
0.0009 \\
0.0000 \\
2.4141-01\end{array}$ \\
\hline $\begin{array}{l}L A-137 \\
L A-138 \\
L A-139 \\
\text { LA-14C } \\
\text { LANTHANUM }\end{array}$ & $\begin{array}{l}0.0000 \\
2.2619-03 \\
2.5572-01 \\
0.0 r 00 \\
2.5798-01\end{array}$ & $\begin{array}{l}0.0000 \\
3.7150-04 \\
4.1662-\pi 2 \\
0.0000 \\
4.2233-02\end{array}$ & $\begin{array}{l}0.0000 \\
2.2619-03 \\
2.5572-01 \\
0.0020 \\
2.5798-01\end{array}$ & $\begin{array}{l}0.0300 \\
5.5055-04 \\
6.1741-02 \\
0.0000 \\
6.2292-02\end{array}$ & $\begin{array}{l}\text { L. 0noo } \\
2.2619-03 \\
2.5572-01 \\
0.0700 \\
2.5798-01\end{array}$ & $\begin{array}{l}0.0000 \\
2.115 \pi-03 \\
2.3718-C 1 \\
0.0000 \\
2.3930-01\end{array}$ \\
\hline $\begin{array}{l}B A-135 \\
B A-136 \\
B A-137 M \\
B A-137 \\
\text { EA-138 } \\
B A-140 \\
B A R 1 \cup M\end{array}$ & $\begin{array}{l}3.2780-03 \\
3.9126-03 \\
0.0000 \\
5.7126-03 \\
3.6421-02 \\
0.0000 \\
4.9324-02\end{array}$ & $\begin{array}{l}5.3827-04 \\
6.4323-04 \\
0.0002 \\
9.3803-04 \\
5.9577-03 \\
0.0000 \\
8.0772-03\end{array}$ & $\begin{array}{l}3.2780-03 \\
3.9126-03 \\
0.0000 \\
5.7126-03 \\
3.6421-02 \\
0.0000 \\
4.9324-02\end{array}$ & $\begin{array}{l}7.9769-04 \\
9.5324-04 \\
0.0000 \\
1.3901-03 \\
8.8290-03 \\
0.0000 \\
1.1970-02\end{array}$ & $\begin{array}{l}3.2780-03 \\
3.9126-03 \\
0.0000 \\
5.7126-03 \\
3.6421-02 \\
0.0300 \\
4.9324-02\end{array}$ & $\begin{array}{l}3.0644-03 \\
3.6619-03 \\
0.0007 \\
5.3403-03 \\
3.3017-02 \\
0.0300 \\
4.5984-72\end{array}$ \\
\hline $\begin{array}{l}C S-133 \\
C S-134 \\
C S-135 \\
C S-136\end{array}$ & $\begin{array}{l}0.0000 \\
0.0000 \\
0.0000 \\
0.0000\end{array}$ & $\begin{array}{l}0.0000 \\
0.0000 \\
0.0000 \\
0.0000\end{array}$ & $\begin{array}{l}0.0000 \\
0.0000 \\
0.0000 \\
0.0000\end{array}$ & $\begin{array}{l}0.0000 \\
0.0000 \\
0.0000 \\
0.0000\end{array}$ & $\begin{array}{l}0.0000 \\
0.0000 \\
0.0000 \\
0.0000\end{array}$ & $\begin{array}{l}0.0000 \\
0.0000 \\
0.0000 \\
0.0000\end{array}$ \\
\hline
\end{tabular}


NUCLIDE WEIGHTS IN GRAMS PER F.E. FOR RELOAO 9

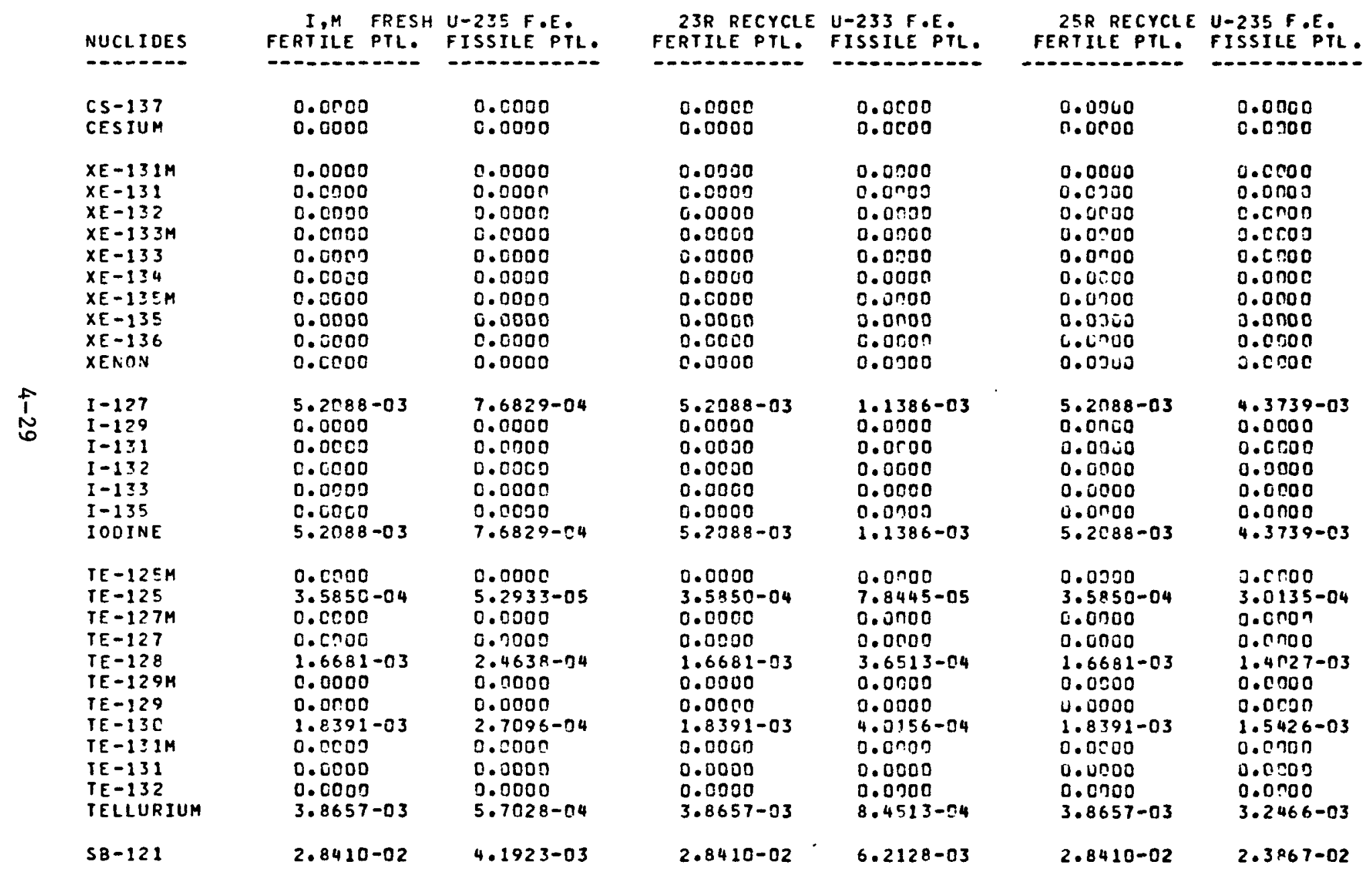


NUCLIDE WEIGHTS IN GRAMS PER F.E. FOR RELOAD?

\begin{tabular}{|c|c|c|c|c|c|c|}
\hline NUCLIDES & $\begin{array}{cl}\text { I,M } & \text { FRESH } \\
\text { FERTILE PTL. }\end{array}$ & $\begin{array}{l}\text { U-235 F.E. } \\
\text { FISSILE PTL. }\end{array}$ & $\begin{array}{l}\text { 23R RECYCLE } \\
\text { FERTILE PTL. }\end{array}$ & $\begin{array}{l}U-233 \text { F E E } \\
\text { FISSILE PTL. }\end{array}$ & $\begin{array}{l}\text { 25R RECYCLE } \\
\text { FERTILE PTL. }\end{array}$ & $\begin{array}{l}\text { U-235 F.E. } \\
\text { FISSILE PTL. }\end{array}$ \\
\hline $\begin{array}{l}S B-122 \\
S B-123 \\
S B-124 \\
S B-125 \\
S B-127 \\
S B-128 \\
S B-129 \\
A N T I M O N Y\end{array}$ & $\begin{array}{l}0.0000 \\
2.1568-.02 \\
0.3000 \\
0.0000 \\
0.0000 \\
0.0000 \\
0.0000 \\
4.9978-02\end{array}$ & $\begin{array}{l}0.0000 \\
3.1793-03 \\
0.0000 \\
0.0000 \\
0.0000 \\
0.0000 \\
0.0000 \\
7.3716-03\end{array}$ & $\begin{array}{l}0.0000 \\
2.1568-02 \\
0.0000 \\
0.0000 \\
0.0000 \\
0.0000 \\
0.0000 \\
4.9978-02\end{array}$ & $\begin{array}{l}0.0000 \\
4.7126-03 \\
0.0000 \\
0.0000 \\
0.0000 \\
0.0000 \\
0.0000 \\
1.0924-02\end{array}$ & $\begin{array}{l}0.0000 \\
2.1568-02 \\
0.0000 \\
0.0000 \\
0.0000 \\
0.0000 \\
0.0000 \\
4.9978-02\end{array}$ & $\begin{array}{l}0.0000 \\
1.8100-02 \\
0.0000 \\
0.0500 \\
0.0000 \\
0.0000 \\
0.0300 \\
4.1967-02\end{array}$ \\
\hline $\begin{array}{l}S N-115 \\
S N-117 M \\
S N-117 \\
S N-119 M \\
S N-119 \\
S N-120 \\
S N-121 M \\
S N-121 \\
S N-123 \\
S N-125 \\
T I N .\end{array}$ & $\begin{array}{l}1.5978-04 \\
0.0000 \\
3.6338-03 \\
0.0000 \\
4.1910-03 \\
1.6138-02 \\
0.0000 \\
0.0000 \\
0.0000 \\
0.0000 \\
2.4123-02\end{array}$ & $\begin{array}{l}2.3622-05 \\
0.0000 \\
5.3728-04 \\
0.0000 \\
6.1245-04 \\
2.3824-03 \\
0.3000 \\
0.0000 \\
0.0000 \\
0.0000 \\
3.5618-03\end{array}$ & $\begin{array}{l}1.5973-04 \\
0.0000 \\
3.6338-03 \\
0.0000 \\
4.1910-03 \\
1.6138-02 \\
0.0000 \\
0.0000 \\
0.0000 \\
0.0300 \\
2.4123-02\end{array}$ & $\begin{array}{l}3.5007-05 \\
0.0000 \\
7.9623-04 \\
0.0000 \\
9.1652-04 \\
3.5306-03 \\
0.3000 \\
0.0000 \\
0.0000 \\
0.0000 \\
5.2784-03\end{array}$ & $\begin{array}{l}1.5978-04 \\
0.0000 \\
3.6338-03 \\
0.0000 \\
4.1910-03 \\
1.6138-02 \\
0.0000 \\
0.0000 \\
0.0000 \\
0.0000 \\
2.4123-02\end{array}$ & $\begin{array}{l}1.3448-04 \\
0.0000 \\
3.0583-03 \\
0.0700 \\
3.5209-03 \\
1.3563-02 \\
0.0000 \\
0.0000 \\
0.0000 \\
0.0000 \\
2.0277-02\end{array}$ \\
\hline $\begin{array}{l}I N-1113 \\
I N-114 \\
I N-115 M \\
\text { IN-1115 } \\
\text { INOIUM }\end{array}$ & $\begin{array}{l}1.7967-02 \\
0.0000 \\
0.0000 \\
4.0843-01 \\
4.2640-01\end{array}$ & $\begin{array}{l}2.6722-04 \\
0.0000 \\
0.0000 \\
6.0715-03 \\
6.3387-03\end{array}$ & $\begin{array}{l}1.7967-02 \\
0.0000 \\
0.0000 \\
4.0843-01 \\
4.2640-01\end{array}$ & $\begin{array}{l}3.9601-04 \\
0.0007 \\
0.0000 \\
8.9977-03 \\
9.3938-03\end{array}$ & $\begin{array}{l}1.7067-02 \\
0.0000 \\
0.0000 \\
4.0843-01 \\
4.2640-01\end{array}$ & $\begin{array}{l}1.5213-03 \\
0.0500 \\
0.0500 \\
3.4566-02 \\
3.6087-02\end{array}$ \\
\hline $\begin{array}{l}C D-11 C \\
C D-111 \\
C D-112 \\
C D-113 \\
C D-114 \\
C D-115 M \\
C D-115 \\
C A D M I U M\end{array}$ & $\begin{array}{l}2.7952-04 \\
6.5155-03 \\
1.2483-02 \\
6.3810-03 \\
1.5060-02 \\
0.0000 \\
0.0000 \\
4.0719-02\end{array}$ & $\begin{array}{l}7.4712-05 \\
7.7833-05 \\
1.4937-04 \\
7.6128-0.5 \\
1.7993-04 \\
0.0000 \\
0.0000 \\
5.5798-04\end{array}$ & $\begin{array}{l}2.7952-04 \\
6.5155-03 \\
1.2483-02 \\
6.3810-03 \\
1.5060-02 \\
0.0000 \\
0.0000 \\
4.0719-02\end{array}$ & $\begin{array}{l}1.1072-04 \\
1.1534-04 \\
2.2136-04 \\
1.1282-04 \\
2.6666-04 \\
0.0000 \\
0.0000 \\
8.2690-04\end{array}$ & $\begin{array}{l}2.7952-04 \\
6.5155-03 \\
1.2483-02 \\
6.3810-03 \\
1.5060-02 \\
0.0500 \\
0.0000 \\
4.0719-02\end{array}$ & $\begin{array}{l}4.2534-04 \\
4.4311-04 \\
8.5036-04 \\
4.3340-04 \\
1.0244-03 \\
0.0000 \\
0.0000 \\
3.1766-03\end{array}$ \\
\hline
\end{tabular}


NUCLIDE NEIGHTS IN GRAMS PER F.E. FOR RELOAD,

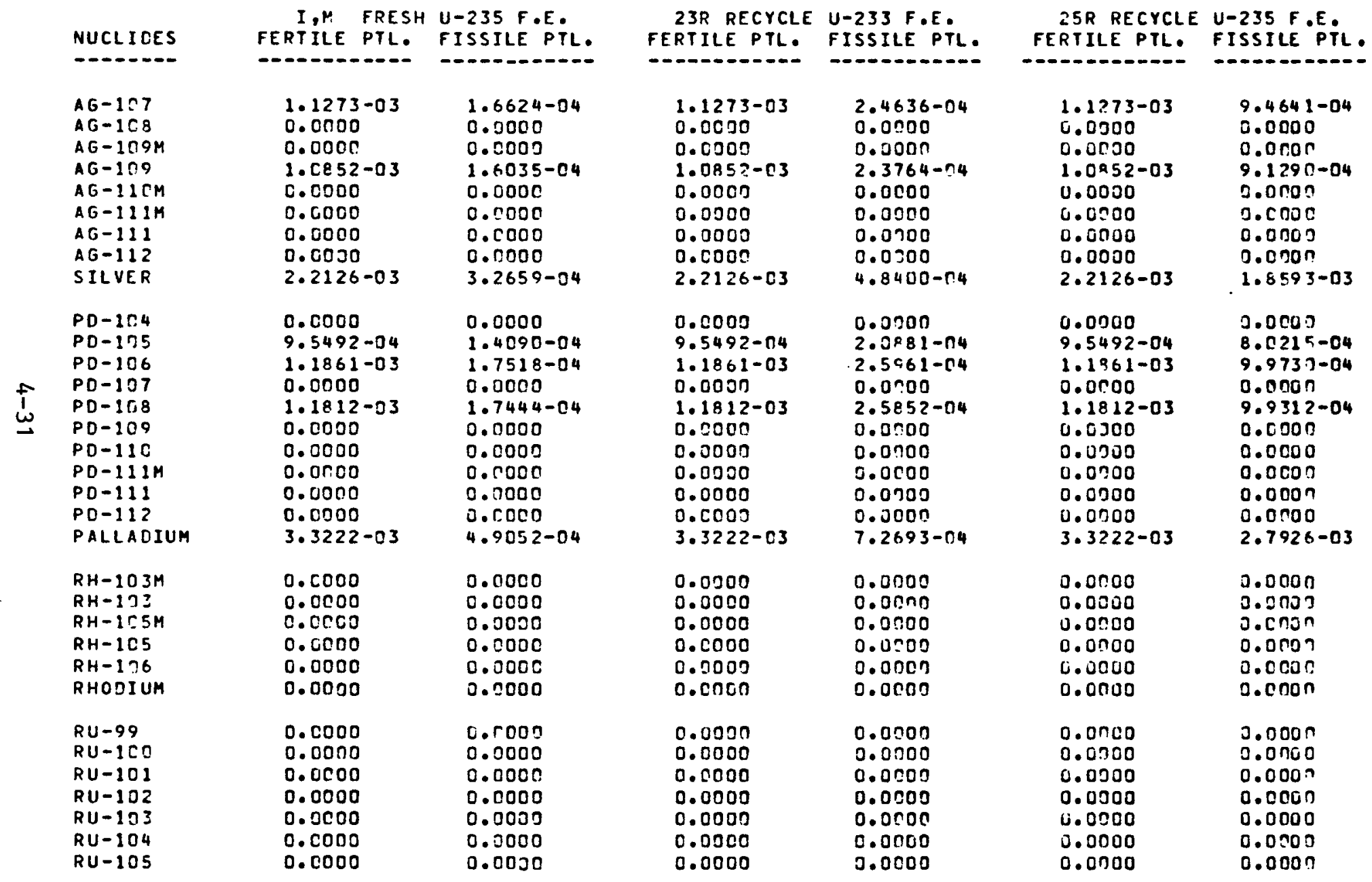


NUCLIDE WEIGHTS IN GRAMS PER F.E. FOR RELOAD 9

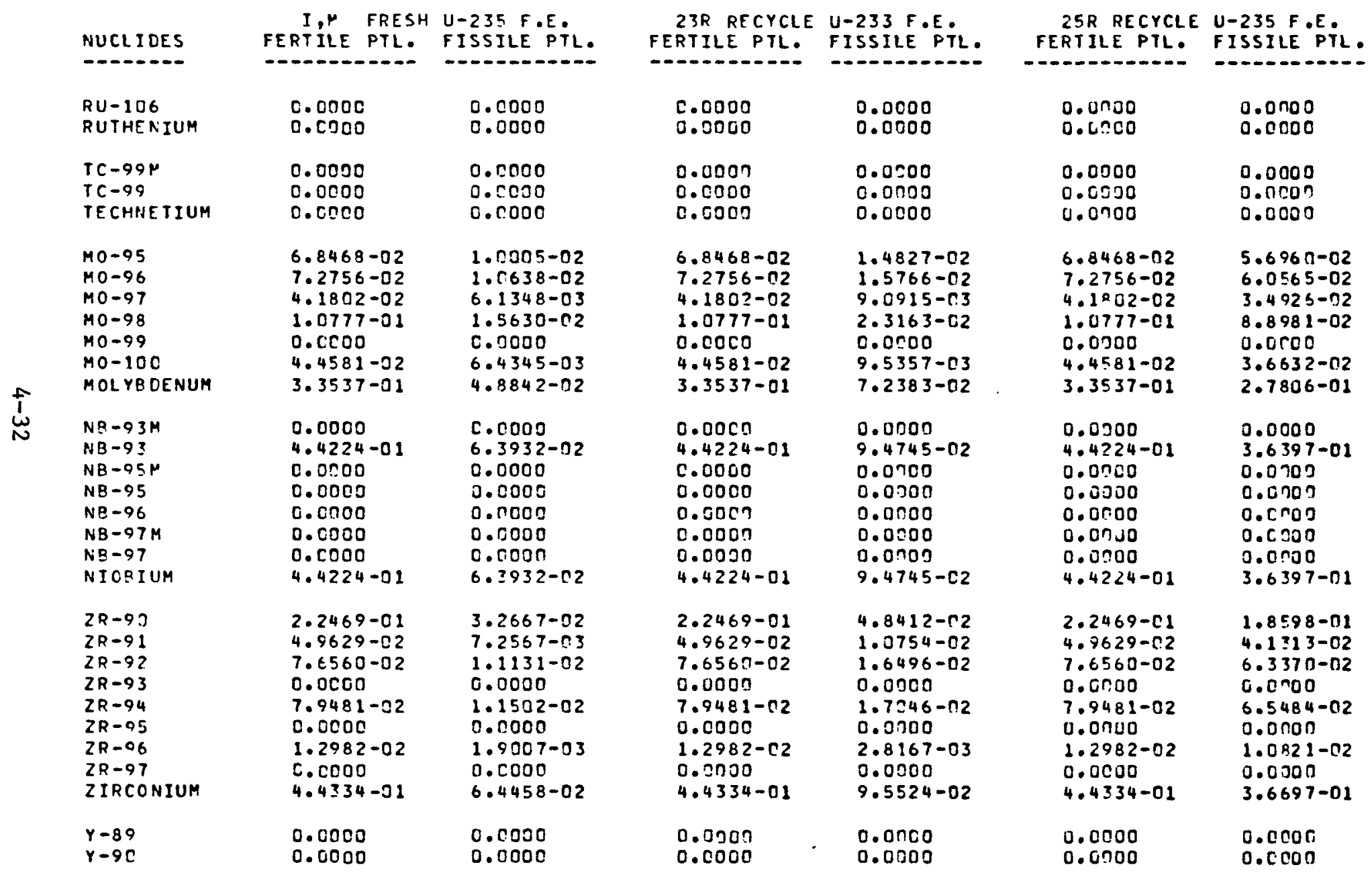


NUCLIDE WEIGHTS IN GRAMS PER F.E. FOR RELOAD

\begin{tabular}{|c|c|c|c|c|c|c|}
\hline NUCLIDES & $\begin{array}{cl}\text { I,M } & \text { FRESH } \\
\text { FERTILE } & \text { PIL. }\end{array}$ & $\begin{array}{l}\text { U-235 F.E. } \\
\text { FISSILE PTL. }\end{array}$ & $\begin{array}{l}\text { 23R RECYCLE } \\
\text { FERTILE PTL. }\end{array}$ & $\begin{array}{l}\text { U-233 F.E. } \\
\text { FISSILE PTL. }\end{array}$ & $\begin{array}{l}\text { 25R RECYCLE } \\
\text { FERTILE PTL. }\end{array}$ & $\begin{array}{l}\text { U-235 F.E. } \\
\text { FISSILE PIL. }\end{array}$ \\
\hline $\begin{array}{l}Y-91 M \\
Y-91 \\
Y-92 \\
Y-93 \\
Y T T E R E I U M\end{array}$ & $\begin{array}{l}0.0000 \\
0.0000 \\
0.0000 \\
0.0000 \\
0.0000\end{array}$ & $\begin{array}{l}0.0000 \\
0.2000 \\
0.0000 \\
0.0000 \\
0.0000\end{array}$ & $\begin{array}{l}0.0000 \\
0.0000 \\
0.0000 \\
0.0000 \\
0.0000\end{array}$ & $\begin{array}{l}0.0000 \\
0.0200 \\
0.0000 \\
0.0000 \\
0.0300\end{array}$ & $\begin{array}{l}0.0000 \\
0.0700 \\
0.0000 \\
0.0300 \\
0.0000\end{array}$ & $\begin{array}{l}0.0 n 00 \\
3.0 \Omega 00 \\
0.0000 \\
0 . c 000 \\
0.0000\end{array}$ \\
\hline $\begin{array}{l}S R-P 6 \\
S R-87 \\
S R-88 \\
S R-89 \\
S R-9 C \\
S R-91 \\
S T R O N T I U M\end{array}$ & $\begin{array}{l}1.7253-02 \\
1.2282-02 \\
1.5039-01 \\
0.0000 \\
0.0000 \\
0.0000 \\
1.7992-01\end{array}$ & $\begin{array}{l}2.5304-03 \\
1.8373-03 \\
2.1730-02 \\
0.0000 \\
0.0000 \\
0.0000 \\
2.6098-02\end{array}$ & $\begin{array}{l}1.7253-02 \\
1.2282-02 \\
1.5039-01 \\
0.7000 \\
0.0000 \\
0.0000 \\
1.7992-01\end{array}$ & $\begin{array}{l}3.7499-03 \\
2.7228-03 \\
3.2203-02 \\
0.0000 \\
0.0000 \\
0.0000 \\
3.8675-02\end{array}$ & $\begin{array}{l}1.7253-02 \\
1.2282-02 \\
1.5039-01 \\
0.0000 \\
0.0030 \\
0.0000 \\
1.7992-01\end{array}$ & $\begin{array}{l}1.4405-02 \\
1.0460-02 \\
1.2371-01 \\
0.0000 \\
3.0005 \\
0.0005 \\
1.4858-01\end{array}$ \\
\hline $\begin{array}{l}R B-25 \\
R B-86 \\
R E-87 \\
\text { RUB ID IUH }\end{array}$ & $\begin{array}{l}0.0005 \\
0.0000 \\
0.0000 \\
0.0000\end{array}$ & $\begin{array}{l}c .0000 \\
0.0000 \\
0.0000 \\
0.0000\end{array}$ & $\begin{array}{l}0.0000 \\
0.0000 \\
0.0300 \\
0.3000\end{array}$ & $\begin{array}{l}0.0000 \\
0.0000 \\
0.0000 \\
0.0000\end{array}$ & $\begin{array}{l}0.0000 \\
0.0700 \\
0.0000 \\
0.0700\end{array}$ & $\begin{array}{l}0.0000 \\
0.0000 \\
3.01000 \\
0.0000\end{array}$ \\
\hline $\begin{array}{l}K R-82 \\
K R-83 \mu \\
K R-83\end{array}$ & $\begin{array}{l}0.0000 \\
0.0000 \\
0.0000\end{array}$ & $\begin{array}{l}0.0000 \\
0.3000 \\
0.0000\end{array}$ & $\begin{array}{l}0.0000 \\
0.0000 \\
0.0000\end{array}$ & $\begin{array}{l}0.0700 \\
0.0200 \\
0.0000\end{array}$ & $\begin{array}{l}0.0000 \\
0.0 .700 \\
0.0000\end{array}$ & $\begin{array}{l}0.0000 \\
0.0000 \\
0.0000\end{array}$ \\
\hline $\begin{array}{l}K R-84 \\
K P-85 \% \\
K R-85\end{array}$ & $\begin{array}{l}0.0000 \\
0.0000 \\
0.0000\end{array}$ & $\begin{array}{l}0.0000 \\
0.0000 \\
0.0000\end{array}$ & $\begin{array}{l}0.0000 \\
0.0000 \\
0.0000\end{array}$ & 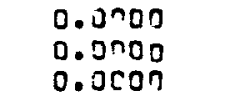 & $\begin{array}{l}0.57 \text { 0 } \\
\text { 0. } \\
0.070000\end{array}$ & $\begin{array}{l}0.0000 \\
0.0 \cap 00 \\
0.0000\end{array}$ \\
\hline $\begin{array}{l}\text { KR-B } 6 \\
\text { KPYPTON }\end{array}$ & $\begin{array}{l}0.0000 \\
0.0000\end{array}$ & $\begin{array}{l}0.0000 \\
0.0000\end{array}$ & $\begin{array}{l}0.000 n \\
0.0000\end{array}$ & $\begin{array}{l}0.0030 \\
0.3000\end{array}$ & $\begin{array}{l}0.0000 \\
0.0500\end{array}$ & $\begin{array}{l}0.0000 \\
0.0000\end{array}$ \\
\hline $\begin{array}{l}B R-79 \\
B R-21 \\
B R-82 \\
B R-83 \\
B R O M I N E\end{array}$ & $\begin{array}{l}0.0000 \\
0.0000 \\
0.0000 \\
0.0000 \\
0.0000\end{array}$ & $\begin{array}{l}0.0000 \\
0.0001 \\
0.0000 \\
0.0000 \\
0.0000\end{array}$ & $\begin{array}{l}0.0000 \\
0.0000 \\
0.0000 \\
0.0000 \\
0.0000\end{array}$ & $\begin{array}{l}0.0000 \\
0.0000 \\
0.0000 \\
0.0500 \\
0.0000\end{array}$ & $\begin{array}{l}0.0000 \\
0.0000 \\
0.0000 \\
0.0000 \\
0.0 n 00\end{array}$ & $\begin{array}{l}0.0000 \\
0.0000 \\
0.0100 \\
0.0700 \\
0.0000\end{array}$ \\
\hline $\begin{array}{l}S E-79 \\
S E-80\end{array}$ & $\begin{array}{l}0.0000 \\
0.0000\end{array}$ & $\begin{array}{l}0.0000 \\
0.0000\end{array}$ & $\begin{array}{l}0.0000 \\
0.0000\end{array}$ & $\begin{array}{l}0.0000 \\
0.0700\end{array}$ & $\begin{array}{l}0.0000 \\
0.0300\end{array}$ & $\begin{array}{l}0.0 n 00 \\
3.0000\end{array}$ \\
\hline
\end{tabular}


NUCLIDE WEIGHTS IN GRAMS PER F.E. FOR RELOAD 9

\begin{tabular}{|c|c|c|c|c|c|c|}
\hline NUCLIDES & $\begin{array}{cl}\text { I,M } & \text { FRESH } \\
\text { FERTILE PTL. }\end{array}$ & $\begin{array}{l}U-235 \text { F.E. } \\
\text { FISSILE PTL. }\end{array}$ & $\begin{array}{l}23 R \text { RECYCLE } \\
\text { FERTILE PTL. }\end{array}$ & $\begin{array}{l}\text { U-233 F.E. } \\
\text { FISSILE PTL. }\end{array}$ & $\begin{array}{l}\text { 25R RECYCLE } \\
\text { FERTILE PTL. }\end{array}$ & $\begin{array}{l}\text { U-235 F.E. } \\
\text { FISSILE PIL. }\end{array}$ \\
\hline------- & $---m-------2$ & 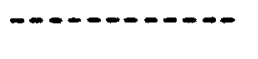 & 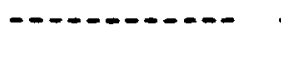 & $-\infty-\infty-\infty-\infty-\infty$ & 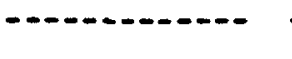 & $--\infty-\infty---\infty-\infty$ \\
\hline $\begin{array}{l}\text { SE-92 } \\
\text { SELENIUM }\end{array}$ & $\begin{array}{l}0.0000 \\
0.0000\end{array}$ & $\begin{array}{l}0.0000 \\
3.0000\end{array}$ & $\begin{array}{l}0.0000 \\
0.0000\end{array}$ & $\begin{array}{l}0.0000 \\
0.0000\end{array}$ & $\begin{array}{l}0.0000 \\
0.0000\end{array}$ & $\begin{array}{l}0.0000 \\
0.0 n 00\end{array}$ \\
\hline $\begin{array}{l}\text { CO-ES } \\
\text { CO-ST } \\
\text { COBALT }\end{array}$ & $\begin{array}{l}0.0000 \\
0.5000 \\
0.0000\end{array}$ & $\begin{array}{l}0.0000 \\
0.0000 \\
0.0000\end{array}$ & $\begin{array}{l}0.0000 \\
0.0000 \\
0.0000\end{array}$ & $\begin{array}{l}0.0000 \\
0.0000 \\
0.0000\end{array}$ & $\begin{array}{l}0.0000 \\
0.0000 \\
0.0000\end{array}$ & $\begin{array}{l}0.0000 \\
0.0000 \\
0.0000\end{array}$ \\
\hline $\begin{array}{l}N I-58 \\
N I-59 \\
N I-6 J \\
N I-61 \\
N I-62 \\
N I-63 \\
N I-64 \\
N I C K E L\end{array}$ & $\begin{array}{l}2.2928+00 \\
0.0000 \\
5.1714-01 \\
2.4928-02 \\
7.4167-02 \\
0.0000 \\
2.4727-02 \\
1.9338+00\end{array}$ & $\begin{array}{l}8.6123-02 \\
0.0005 \\
3.4647-02 \\
1.6774-03 \\
4.9441-03 \\
0.0300 \\
1.6367-03 \\
1.2903-01\end{array}$ & $\begin{array}{l}1.2928+00 \\
0.0007 \\
5.1714-01 \\
2.4928-02 \\
7.4167-02 \\
0.0000 \\
2.4727-02 \\
1.9338+00\end{array}$ & $\begin{array}{l}1.2763-01 \\
0.0000 \\
5.1346-02 \\
2.4858-53 \\
7.3270-03 \\
0.0000 \\
2.4255-03 \\
1.9122-01\end{array}$ & $\begin{array}{l}1.2928+00 \\
0.0500 \\
5.1714-01 \\
2.4928-02 \\
7.4167-02 \\
0.0000 \\
2.4727-02 \\
1.9738+00\end{array}$ & $\begin{array}{l}4.9031-01 \\
0.0700 \\
1.9725-01 \\
9.5494-03 \\
2.8147-02 \\
0.0700 \\
9.3177-03 \\
7.3457-01\end{array}$ \\
\hline $\begin{array}{l}C L-35 \\
C L-36 \\
C L-37 \\
C H L O R \text { INE }\end{array}$ & $\begin{array}{l}2.3925-01 \\
0.0000 \\
8.2474-02 \\
3.2172-01\end{array}$ & $\begin{array}{l}0.0000 \\
0.000 n \\
0.0000 \\
0.0000\end{array}$ & $\begin{array}{l}2.3925-01 \\
0.0000 \\
8.2474-02 \\
3.2172-01\end{array}$ & $\begin{array}{l}0.00 .00 \\
0.0000 \\
0.0000 \\
0.0000\end{array}$ & $\begin{array}{l}2.3925-01 \\
0.0000 \\
8.2474-02 \\
3.2172-01\end{array}$ & $\begin{array}{l}0.0 \cos \\
0.0 \cos \\
0.0 \cos \\
0.0000\end{array}$ \\
\hline $\begin{array}{l}s-32 \\
s-33 \\
s-34 \\
s-35 \\
s-36 \\
S U L F U R\end{array}$ & $\begin{array}{l}1.9497+01 \\
1.6183-01 \\
9.0944-01 \\
0.0000 \\
3.2098-03 \\
2.0571+01\end{array}$ & $\begin{array}{l}6.4235-02 \\
5.3538-04 \\
3.0479-03 \\
0.0000 \\
1.0889-06 \\
6.7819-02\end{array}$ & $\begin{array}{l}1.9497+01 \\
1.6183-01 \\
9.0944-01 \\
0.0000 \\
3.2098-03 \\
2.0571+01\end{array}$ & $\begin{array}{l}9.5194-02 \\
7.9342-n 4 \\
4.5168-03 \\
0.3000 \\
1.6137-06 \\
1.0051-01\end{array}$ & $\begin{array}{l}1.9497+01 \\
1.6183-01 \\
9.0944-01 \\
0.0000 \\
3.2098-03 \\
2.0571+01\end{array}$ & $\begin{array}{l}3.6569-01 \\
3.0480-03 \\
1.7352-02 \\
0.0000 \\
6.1993-06 \\
3.8610-01\end{array}$ \\
\hline $\begin{array}{l}P-33 \\
\text { PHOSPHOROUS }\end{array}$ & $\begin{array}{l}0.0000 \\
0.0900\end{array}$ & $\begin{array}{l}0.0000 \\
0.0000\end{array}$ & $\begin{array}{l}0.0000 \\
0.0000\end{array}$ & $\begin{array}{l}0.0000 \\
0.0700\end{array}$ & $\begin{array}{l}0.0000 \\
0.0000\end{array}$ & $\begin{array}{l}0.0000 \\
0.0000\end{array}$ \\
\hline SILICON & 0.0000 & $2.0326+03$ & 0.0000 & $3.0123+03$ & 0.0000 & $1.1572+04$ \\
\hline $\begin{array}{l}0-16 \\
0-17 \\
O X Y \in E N\end{array}$ & $\begin{array}{l}1.1888+03 \\
4.6735-01 \\
4.180809 \\
1.1893+03\end{array}$ & $\begin{array}{l}4.3997+01 \\
1.7296-02 \\
4.4014+01\end{array}$ & $\begin{array}{l}1.1888+03 \\
4.6735-01 \\
1.188+05 \\
1.1895+03\end{array}$ & $\begin{array}{l}6.5201+C 1 \\
2.5632-02 \\
6.5227+01\end{array}$ & $\begin{array}{l}1.1888+03 \\
4.6735-01 \\
1.1893+03\end{array}$ & $\begin{array}{l}2.5048+02 \\
9.8468-02 \\
2.5057+02\end{array}$ \\
\hline
\end{tabular}


NUCLIDE WEIGHTS IN GRAMS PER F.E. FOR RELOAD 9

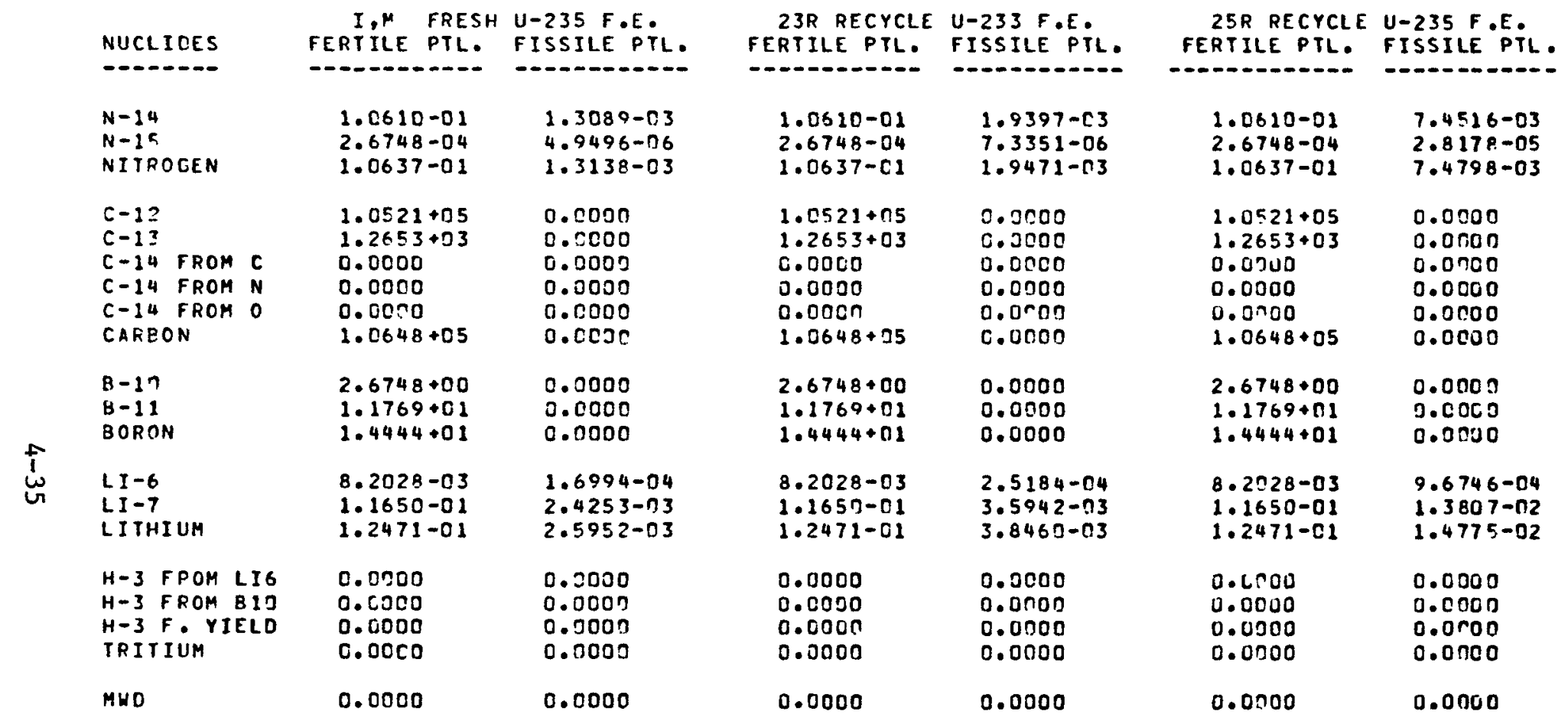


NUCLIDE ACIIVITIES IN CURICS PER F.E. FOR RELOAD

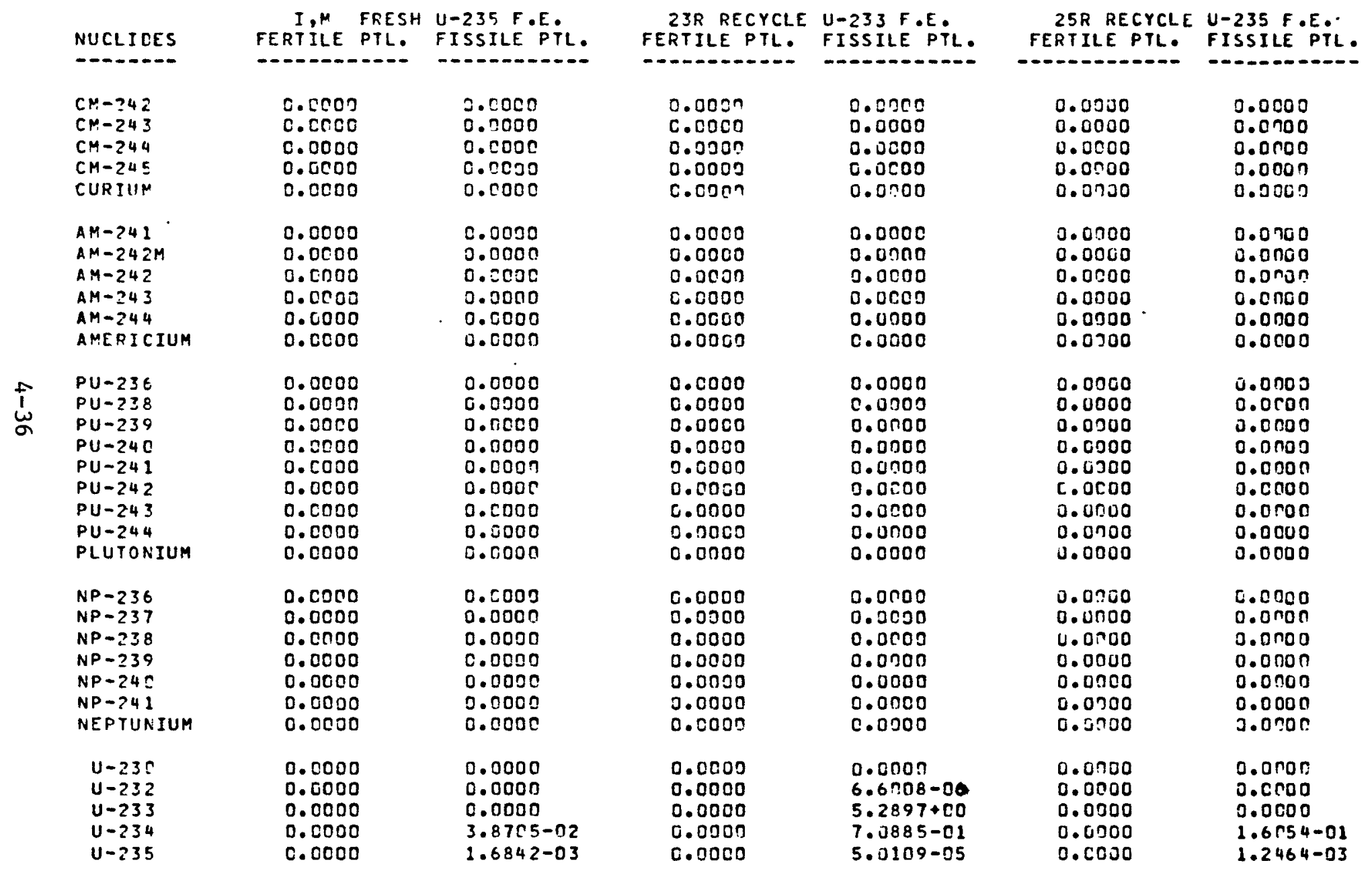


THESE ARE THE CONCENTRATIONS AS LOADED FOR RELOAD

NUCLIDE ACTIVITIES IN CURIES PER F.E. FOR RELOAD 9

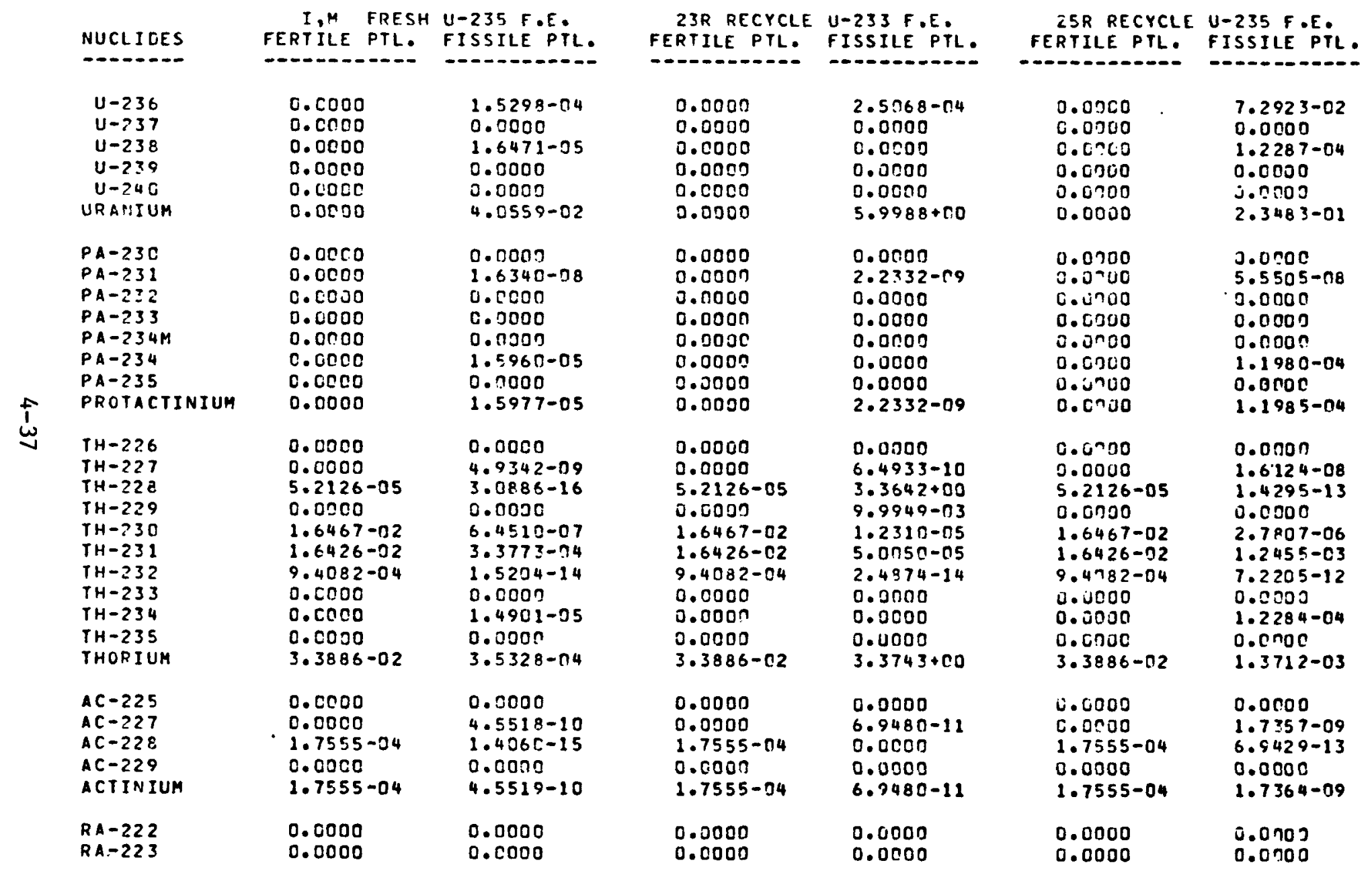


NUCLIDE ACTIVITIES IN CURIES PER F.E. FOR RELOAD 9

\begin{tabular}{|c|c|c|c|c|c|c|}
\hline NUCLIDES & $\begin{array}{cl}\text { I,M } & \text { FRESH } \\
\text { FERTILE PTL. }\end{array}$ & $\begin{array}{l}U-2 \geq 5 \quad F \cdot E \\
F I S S I L E \text { PTL. }\end{array}$ & $\begin{array}{l}\text { 2₹R RECYCLE } \\
\text { FERTILE PTL. }\end{array}$ & $\begin{array}{l}\text { U-233 F.E. } \\
\text { FISSILE PTL. }\end{array}$ & $\begin{array}{l}\text { 25R RECYCLE } \\
\text { FERTILE PTL. }\end{array}$ & $\begin{array}{l}\text { U- } 235 \text { F } . E \text {. } \\
\text { FISSILE PTL. }\end{array}$ \\
\hline $\begin{array}{l}\text { RA }-224 \\
R A-225 \\
\text { RA-226 } \\
\text { RA-227 } \\
\text { RA-226 } \\
\text { RA-229 } \\
\text { RADIUM }\end{array}$ & $\begin{array}{l}5.1377-05 \\
0.0000 \\
1.4261-05 \\
0.0000 \\
1.7587-03 \\
0.0000 \\
1.8244-03\end{array}$ & $\begin{array}{l}0.0003 \\
0.0000 \\
2.8228-10 \\
0.000 \mathrm{C} \\
1.4677-14 \\
0.000 \mathrm{C} \\
2.8229-10\end{array}$ & $\begin{array}{l}5.1377-05 \\
0.0000 \\
1.4261-05 \\
0.0000 \\
1.7587-03 \\
0.0000 \\
1.8244-03\end{array}$ & $\begin{array}{l}0.0-00 \\
0.0000 \\
5.3290-09 \\
0.0000 \\
2.3925-14 \\
0.0000 \\
5.3291-09\end{array}$ & $\begin{array}{l}5.1277-05 \\
0.0000 \\
1.4261-05 \\
0.0000 \\
1.7587-03 \\
0.0000 \\
1.8244-03\end{array}$ & $\begin{array}{l}0.0000 \\
0.0 n 00 \\
1.2028-09 \\
3.0000 \\
6.9693-12 \\
9.0 \cap 00 \\
1.2098-09\end{array}$ \\
\hline $\begin{array}{l}F R-221 \\
F R-223 \\
F R-224 \\
F R A N C I U M\end{array}$ & $\begin{array}{l}0.0000 \\
0.0000 \\
0.0000 \\
0.0000\end{array}$ & $\begin{array}{l}0.0000 \\
0.7000 \\
0.0000 \\
0.0000\end{array}$ & $\begin{array}{l}0.0000 \\
0.0000 \\
0.0000 \\
0.0000\end{array}$ & $\begin{array}{l}0.0700 \\
0.0700 \\
0.0000 \\
0.0000\end{array}$ & $\begin{array}{l}0.0000 \\
0.0 n 00 \\
0.0000 \\
0.0000\end{array}$ & $\begin{array}{l}0.0 \operatorname{con} \pi \\
0 . \cos 0 \\
0.0 \cos \\
0.0 \cos \end{array}$ \\
\hline $\begin{array}{l}R N-218 \\
R N-219 \\
R N-220 \\
R N-222 \\
R N-223 \\
R N-224 \\
R A D O N\end{array}$ & $\begin{array}{l}0.0000 \\
0.0000 \\
5.1228-05 \\
1.4141-05 \\
0.0000 \\
0.0000 \\
6.5370-05\end{array}$ & $\begin{array}{l}0.2000 \\
0.0000 \\
0.0000 \\
0.0000 \\
0.0000 \\
0.0000 \\
0.0000\end{array}$ & $\begin{array}{l}0.000 \Omega \\
0.0000 \\
5.1228-05 \\
1.4141-05 \\
0.0000 \\
0.0000 \\
6.5370-05\end{array}$ & $\begin{array}{l}0.0000 \\
0.0000 \\
0.0000 \\
0.0000 \\
0.0000 \\
0.0000 \\
0.4000\end{array}$ & $\begin{array}{l}0.0000 \\
0.0003 \\
5.1228-05 \\
1.4141-05 \\
0.0000 \\
0.0000 \\
6.5370-05\end{array}$ & $\begin{array}{l}0.0 \text { nod } \\
0.0000 \\
0 . c r 00 \\
0 . c \text { non } \\
0.0 \text { con } \\
0.0 \text { roo } \\
0.0 \text { noo }\end{array}$ \\
\hline $\begin{array}{l}\text { AT }-217 \\
\text { AT }-218 \\
\text { AT }-219 \\
\text { ASTAT INE }\end{array}$ & $\begin{array}{l}0.0000 \\
0.0000 \\
0.0000 \\
0.0000\end{array}$ & $\begin{array}{l}0.0000 \\
0.0000 \\
0.0000 \\
0.0000\end{array}$ & $\begin{array}{l}0.0000 \\
0.0001 \\
0.0000 \\
0.0000\end{array}$ & $\begin{array}{l}0.0000 \\
0.0500 \\
0.0000 \\
0.0200\end{array}$ & $\begin{array}{l}0.0000 \\
0.0000 \\
0.0000 \\
0.0000\end{array}$ & 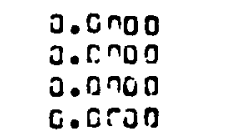 \\
\hline $\begin{array}{l}P O-2 C 9 \\
P O-21 C \\
P O-211 \\
P O-212 \\
P O-213 \\
P O-214 \\
P O-215 \\
P O-216 \\
P O-218 \\
P O L O N I U M\end{array}$ & $\begin{array}{l}0.0000 \\
2.6619-07 \\
0.0000 \\
3.1886-05 \\
0.0000 \\
1.4047-05 \\
0.0000 \\
5.1080-05 \\
1.4117-05 \\
1.1140-04\end{array}$ & $\begin{array}{l}0.0000 \\
0.0000 \\
0.0000 \\
0.0000 \\
0.0000 \\
0.0000 \\
0.0000 \\
0.0000 \\
0.0000 \\
0.0000\end{array}$ & $\begin{array}{l}0.0000 \\
2.6619-07 \\
0.0000 \\
3.1886-05 \\
0.0000 \\
1.4047-05 \\
0.0000 \\
5.1080-05 \\
1.4117-05 \\
1.1140-04\end{array}$ & $\begin{array}{l}0.0000 \\
0.0000 \\
0.0 \times 00 \\
0.0000 \\
0.0005 \\
0.0000 \\
0.0000 \\
0.0000 \\
0.0300 \\
0.0000\end{array}$ & $\begin{array}{l}0.0000 \\
2.6619-07 \\
0.0000 \\
3.1586-05 \\
0.0000 \\
1.4047-05 \\
0.0700 \\
5.1080-05 \\
1.4117-05 \\
1.1140-04\end{array}$ & $\begin{array}{l}0.0000 \\
0.0 n 00 \\
0.0 \sim 00 \\
0.0000 \\
0.0000 \\
0.0050 \\
0.0000 \\
0.0000 \\
0.0000 \\
0.0000\end{array}$ \\
\hline
\end{tabular}


NUCLIDE ACTIVITIES IN CURIES PER F.E. FOR RELOAD 9

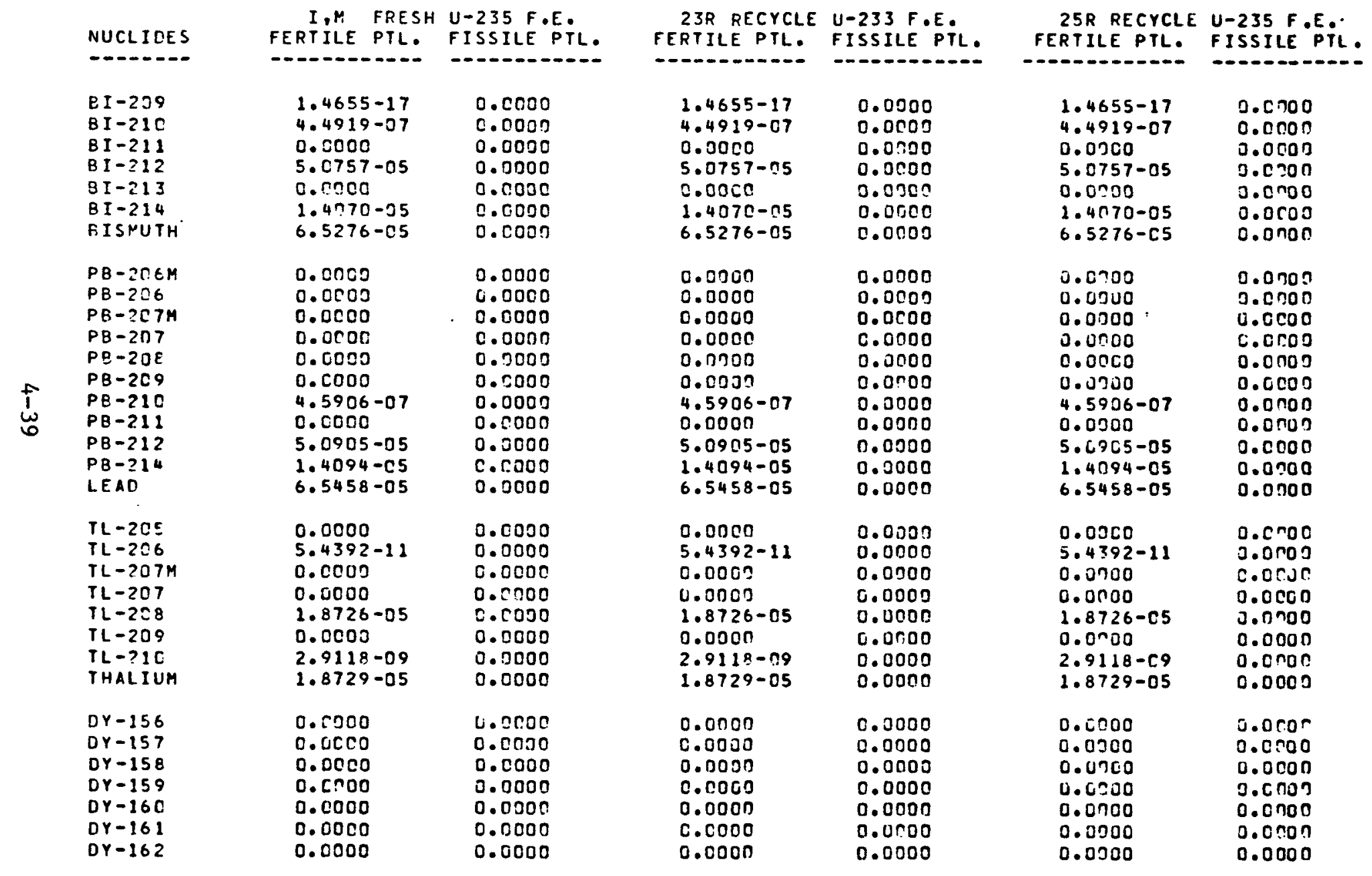


NUCLIDE ACTIVITIES IN CURIES PER F.E. FOR RELOAD 9

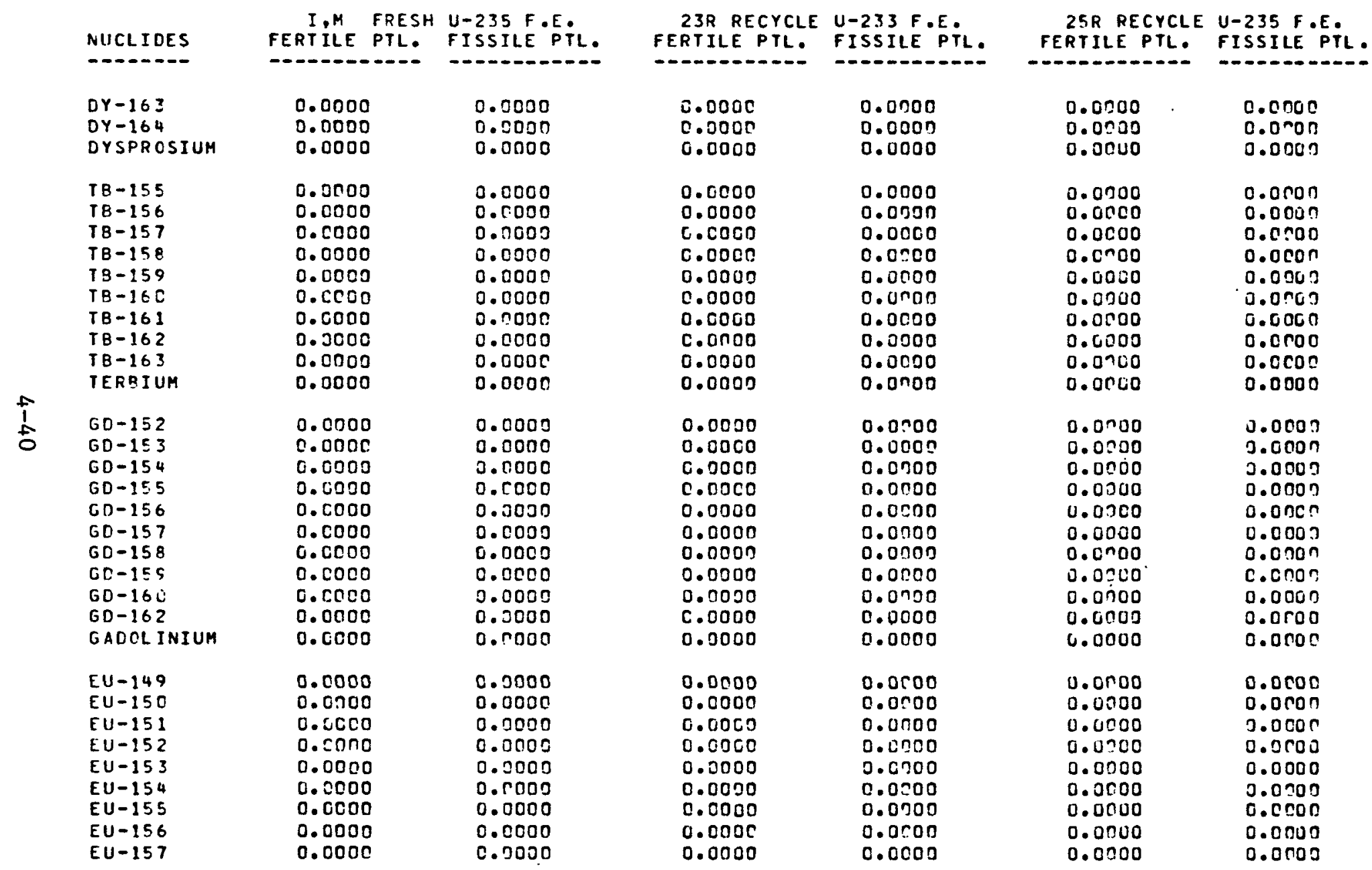


NUCLIDE ACTIVITIES IN CURIES PER F.E. FOR RELOAD 9

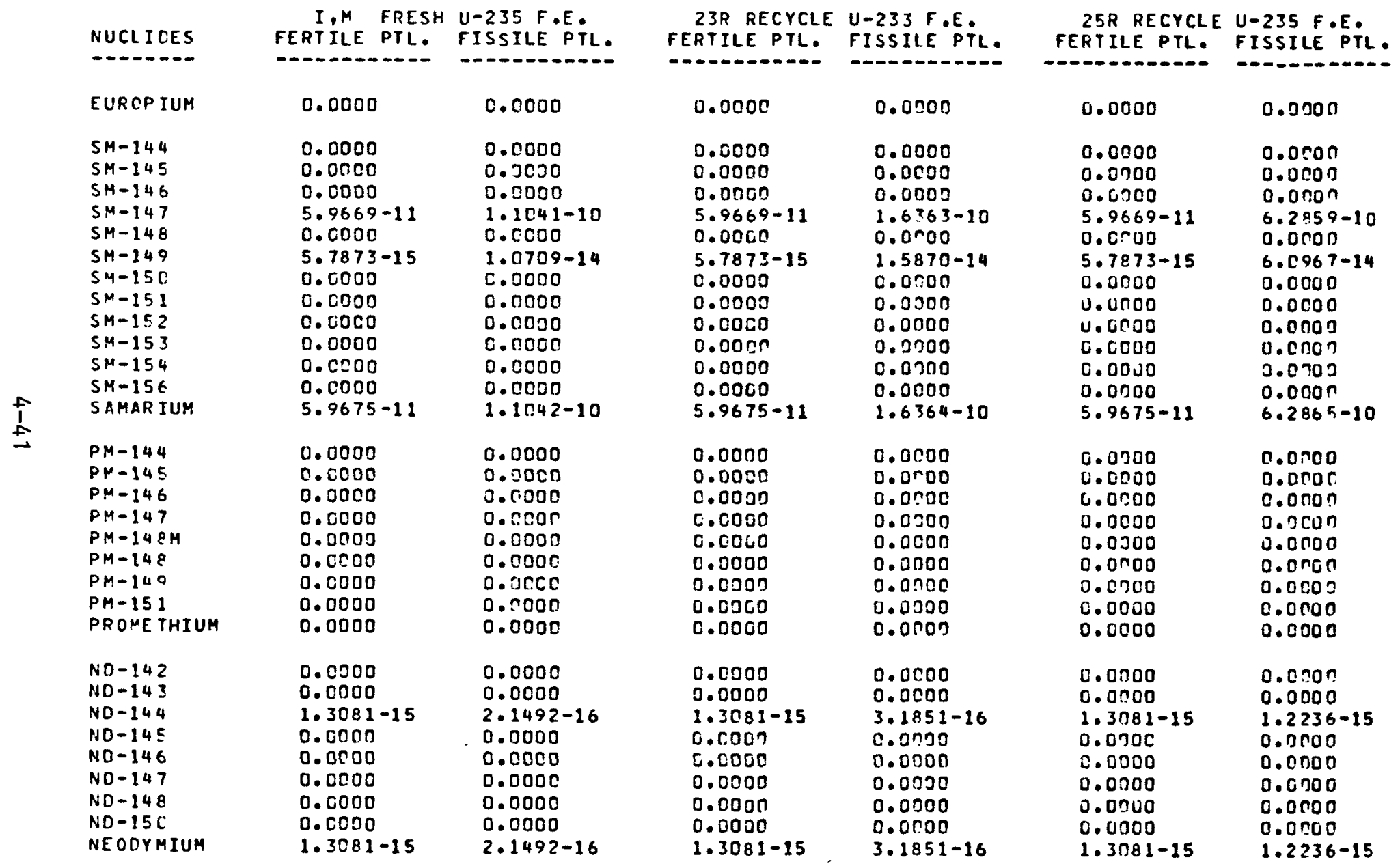


NUCLIDE ACTIVITIES IN CURIES PER F.E. FOR RELOAD?

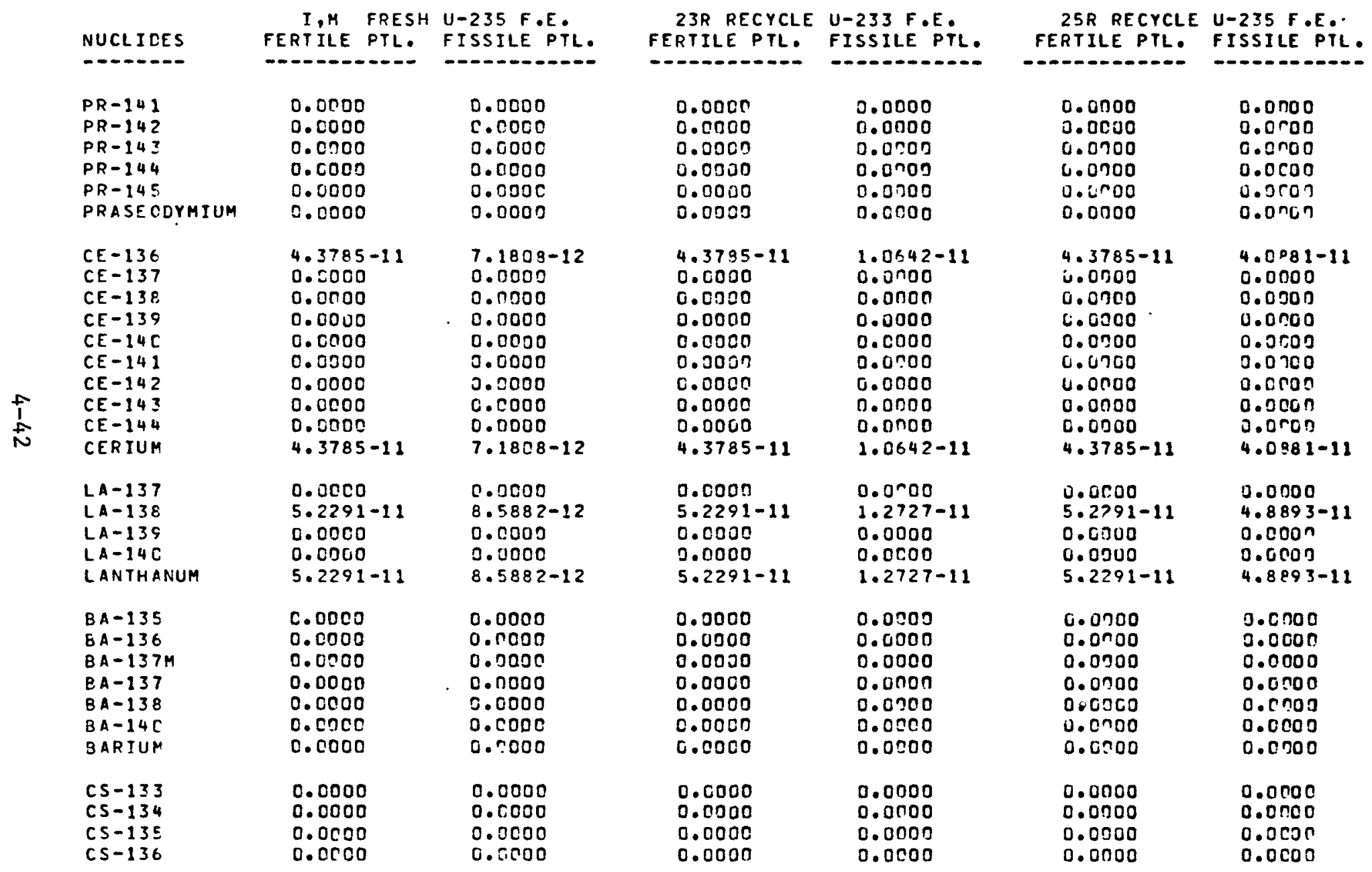


NUCLIDE ACTIVITIES IN CURIES PER F.E. FOR RELOAD 9

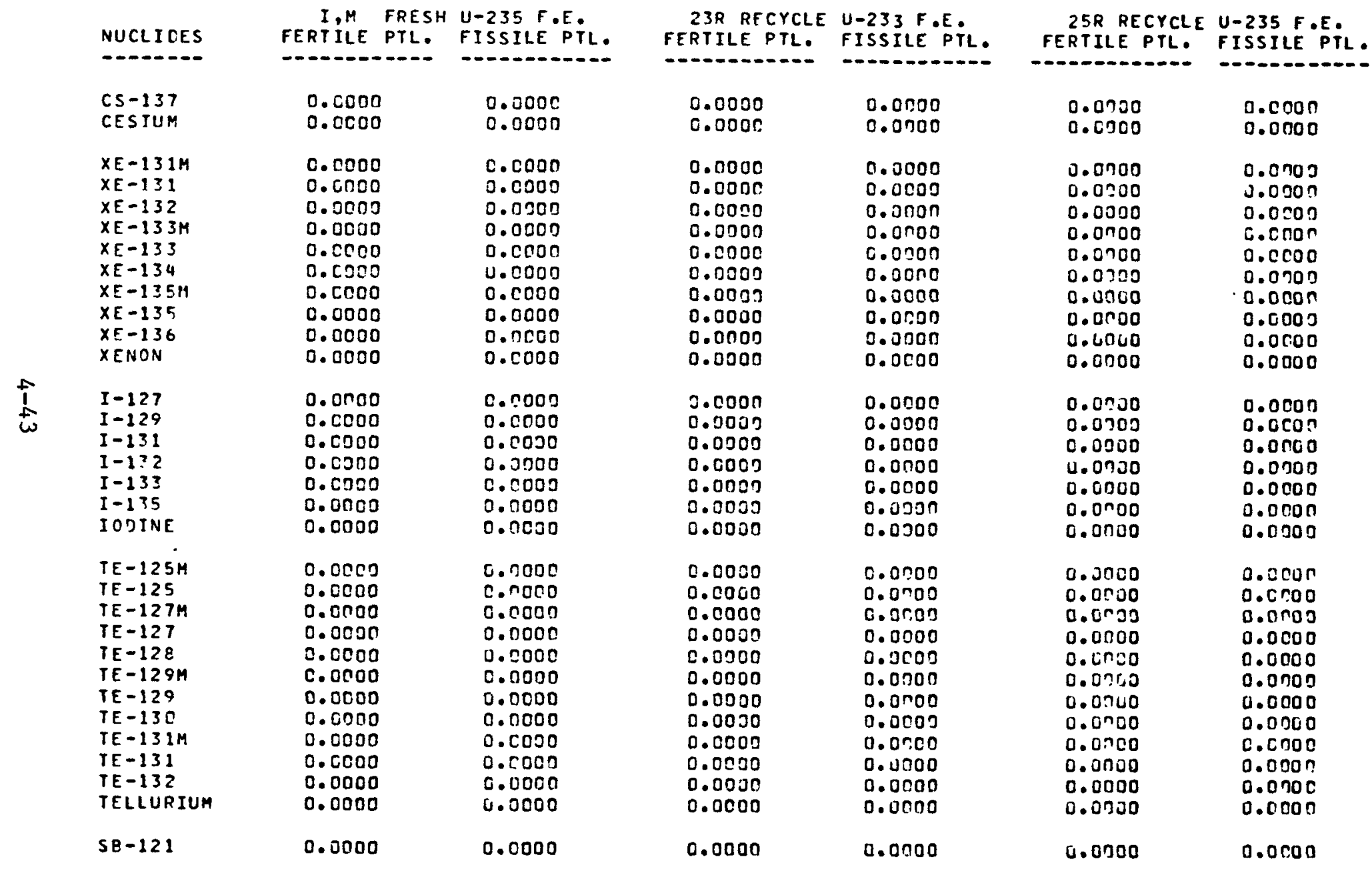


NUCLIDE ACTIVIIIES IN CURIES PER F.E. FOR RELOAD

\begin{tabular}{|c|c|c|c|c|c|c|}
\hline NUCLIOES & $\begin{array}{l}\text { I, M FRESH } \\
\text { FERTILE PTL. }\end{array}$ & $\begin{array}{l}\text { U-235 F.E. } \\
\text { FISSILE PTL. }\end{array}$ & $\begin{array}{l}\text { 23R RECYCLE } \\
\text { FERTILE PTL. }\end{array}$ & $\begin{array}{l}\text { U-233 F.E. } \\
\text { FISSILE PTL. }\end{array}$ & $\begin{array}{l}\text { 25R RECYCLE } \\
\text { FERTILE PTL. }\end{array}$ & $\begin{array}{l}\text { U-235 F PE } \\
\text { FISSILE PTL. }\end{array}$ \\
\hline $\begin{array}{l}S B-122 \\
S B-123 \\
S B-124 \\
S B-125 \\
S E-127 \\
S B-128 \\
S B-129 \\
\text { ANTIMONY }\end{array}$ & $\begin{array}{l}0.0000 \\
0.0000 \\
0.0000 \\
0.0000 \\
0.0000 \\
0.0000 \\
0.0000 \\
0.0000\end{array}$ & $\begin{array}{l}0.0007 \\
0.0000 \\
0.0000 \\
0.0000 \\
0.0000 \\
0.0000 \\
0.0000 \\
0.0000\end{array}$ & $\begin{array}{l}0.0002 \\
0.0000 \\
0.0000 \\
0.0000 \\
0.0000 \\
0.0000 \\
0.0000 \\
0.0000\end{array}$ & $\begin{array}{l}0.0000 \\
0.0000 \\
c .0000 \\
0.0000 \\
0.0000 \\
0.0000 \\
C .0000 \\
0.0000\end{array}$ & $\begin{array}{l}0.0000 \\
0.0000 \\
0.0000 \\
0.0000 \\
0.0700 \\
0.0030 \\
0.0000 \\
0.0000\end{array}$ & $\begin{array}{l}0.0000 \\
0.000 n \\
0.0000 \\
0.0000 \\
0.0700 \\
0.000 n \\
0.0000 \\
0.0000\end{array}$ \\
\hline $\begin{array}{l}S N-115 \\
S N-117 M \\
S N-117 \\
S N-119 M \\
S N-119 \\
S N-12 C \\
S N-121 M \\
S N-121 \\
S N-123 \\
S N-125 \\
T I N\end{array}$ & $\begin{array}{l}0.0000 \\
0.0000 \\
0.0000 \\
0.0000 \\
0.0000 \\
0.0000 \\
0.0000 \\
0.0000 \\
0.0000 \\
0.0000 \\
0.0000\end{array}$ & $\begin{array}{l}0.2000 \\
0.0000 \\
0.0000 \\
0.0300 \\
0.0000 \\
0.2000 \\
0.1000 \\
0.0000 \\
0.0000 \\
0.0000 \\
0.0000\end{array}$ & $\begin{array}{l}0.0000 \\
0.0000 \\
0.0000 \\
0.0000 \\
0.0000 \\
0.0000 \\
0.0000 \\
0.0000 \\
0.0000 \\
0.0000 \\
0.3000\end{array}$ & $\begin{array}{l}0.0000 \\
0.0350 \\
0.0000 \\
0.0000 \\
0.0000 \\
0.0500 \\
0.0000 \\
0.0000 \\
0.0 \times 00 \\
0.0000 \\
0.0500\end{array}$ & $\begin{array}{l}0.0000 \\
0.0700 \\
0.0200 \\
0.0000 \\
0.0000 \\
0.0700 \\
0.0300 \\
0.0000 \\
0.0000 \\
0.0000 \\
0.0030\end{array}$ & $\begin{array}{l}0.0000 \\
0.0000 \\
0.0 n 00 \\
0.0 n 00 \\
0.0 n 00 \\
0.0000 \\
0.0 \operatorname{con} \\
0.0 \operatorname{con} \\
0.0000 \\
0.000 n \\
0.0000\end{array}$ \\
\hline 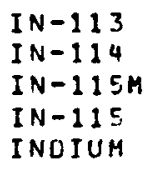 & $\begin{array}{l}0.0000 \\
0.0000 \\
0.0000 \\
0.0000 \\
0.0000\end{array}$ & $\begin{array}{l}0.0000 \\
0.0000 \\
0.3000 \\
0.0000 \\
0.0000\end{array}$ & $\begin{array}{l}0.0000 \\
0.0000 \\
0.0000 \\
0.0000 \\
0.0000\end{array}$ & $\begin{array}{l}0.0000 \\
0.0300 \\
C .0000 \\
0.0000 \\
0.0000\end{array}$ & $\begin{array}{l}0.0000 \\
0.0000 \\
0.0000 \\
0.0000 \\
0.0000\end{array}$ & $\begin{array}{l}0.0000 \\
0.0000 \\
0.0000 \\
0.0000 \\
0.0000\end{array}$ \\
\hline $\begin{array}{l}C D-1110 \\
C D-111 \\
C D-112 \\
C D-113 \\
C D-114 \\
C D-115 M \\
C D-115 \\
C A D M I \cup M\end{array}$ & $\begin{array}{l}0.0000 \\
0.0000 \\
0.0000 \\
0.0000 \\
0.0000 \\
0.0000 \\
0.0000 \\
0.0000\end{array}$ & $\begin{array}{l}0.0000 \\
0.0000 \\
0.0000 \\
0.0000 \\
0.0000 \\
0.0000 \\
0.0000 \\
0.0000\end{array}$ & $\begin{array}{l}0.0000 \\
0.0000 \\
0.0000 \\
0.0000 \\
0.0000 \\
0.0000 \\
0.0000 \\
0.0000\end{array}$ & $\begin{array}{l}0.0000 \\
0.0 \text { 000 } \\
0.0000 \\
0.050 .3 \\
0.0000 \\
0.0000 \\
0.0000 \\
0.0000\end{array}$ & $\begin{array}{l}0.0000 \\
0.0 n 50 \\
0.0000 \\
0.0 n 00 \\
0.0 n 00 \\
0.0000 \\
0.0000 \\
0.0 \text {. } 00\end{array}$ & $\begin{array}{l}0 . c 000 \\
0.0000 \\
0.0000 \\
0.0000 \\
0.0000 \\
0.0000 \\
0.0000 \\
0.0000\end{array}$ \\
\hline
\end{tabular}


NUCLIDE ACTIVITIES IN CURIES PER F.E. FOR RELOAD 9

\begin{tabular}{|c|c|c|c|c|c|c|}
\hline NUCLIICES & $\begin{array}{l}\text { IPM FRESH } \\
\text { FERTILE PTL. }\end{array}$ & $\begin{array}{l}\text { U-235 F.E. } \\
\text { FISSILE PTL. }\end{array}$ & $\begin{array}{l}\text { 23R RECYCLE } \\
\text { FERTILE PTL. }\end{array}$ & $\begin{array}{l}\text { U-233 F.E. } \\
\text { FISSILE PTL. }\end{array}$ & $\begin{array}{l}\text { 25R RECYCLE } \\
\text { FERTILE PTL: }\end{array}$ & $\begin{array}{l}\text { U-235 F EE. } \\
\text { FISSILE PTL. }\end{array}$ \\
\hline $\begin{array}{l}A G-107 \\
A G-108 \\
A G-109 M \\
A G-109 \\
A G-11: M \\
A G-111 M \\
A G-111 \\
A G-112 \\
S I L V E R\end{array}$ & $\begin{array}{l}0.0000 \\
0.0000 \\
0.0000 \\
0.0000 \\
0.0000 \\
0.0000 \\
0.0000 \\
0.0000 \\
0.0000\end{array}$ & $\begin{array}{l}0.2000 \\
0.0000 \\
0.0000 \\
0.0000 \\
0.0000 \\
0.0000 \\
0.0000 \\
0.0000 \\
0.0000\end{array}$ & $\begin{array}{l}0.0000 \\
0.0000 \\
0.0000 \\
0.0000 \\
0.0000 \\
0.0000 \\
0.0000 \\
0.0000 \\
0.0000\end{array}$ & $\begin{array}{l}0.0000 \\
0.0 n 05 \\
0.0000 \\
0.0000 \\
0.0000 \\
0.0000 \\
0.0000 \\
0.0000 \\
0.0000\end{array}$ & $\begin{array}{l}0.0000 \\
0.0000 \\
0.0000 \\
0.0000 \\
0.0000 \\
0.0000 \\
0.0000 \\
0.0200 \\
0.0000\end{array}$ & $\begin{array}{l}0.0000 \\
0.0500 \\
0.0000 \\
0.0000 \\
0.0005 \\
0.0000 \\
0.0000 \\
0.0000 \\
0.0000\end{array}$ \\
\hline $\begin{array}{l}P D-104 \\
P D-105 \\
P D-106 \\
P D-107 \\
P D-108 \\
P D-109 \\
P D-110 \\
P D-111 M \\
P D-111 \\
P D-112 \\
P A L L A D I U M\end{array}$ & $\begin{array}{l}0.0000 \\
0.0000 \\
0.0000 \\
0.0000 \\
0.0000 \\
0.0000 \\
0.0000 \\
0.0000 \\
0.0000 \\
0.0000 \\
0.0000\end{array}$ & $\begin{array}{l}0.0000 \\
0.0000 \\
0.0000 \\
0.0000 \\
0.0000 \\
0.0000 \\
0.0000 \\
0.0000 \\
0.0000 \\
0.0000 \\
0.0000\end{array}$ & $\begin{array}{l}0.0000 \\
0.0000 \\
0.0000 \\
0.0000 \\
0.0000 \\
0.0000 \\
0.0000 \\
0.0000 \\
0.0000 \\
0.0000 \\
0.0000\end{array}$ & $\begin{array}{l}0.0300 \\
0.0000 \\
0.0000 \\
0.0000 \\
0.0000 \\
0.0030 \\
0.0000 \\
0.0000 \\
0.0000 \\
0.0000 \\
0.0000\end{array}$ & $\begin{array}{l}0.0700 \\
0.0300 \\
0.0000 \\
0.0000 \\
0.0500 \\
0.0500 \\
0.0500 \\
0.0000 \\
0.0000 \\
0.0300 \\
0.0700\end{array}$ & $\begin{array}{l}0.0000 \\
0.0000 \\
0.0000 \\
0.0000 \\
0.0000 \\
0.0000 \\
0.0000 \\
0.0000 \\
0.0000 \\
0.0000 \\
0.0000\end{array}$ \\
\hline 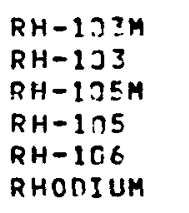 & $\begin{array}{l}0.0000 \\
0.0000 \\
0.0000 \\
0.0000 \\
0.0000 \\
0.0000\end{array}$ & $\begin{array}{l}0.0000 \\
0.0000 \\
0.0000 \\
0.0000 \\
0.0000 \\
0.0000\end{array}$ & $\begin{array}{l}0.0000 \\
0.0000 \\
0.0003 \\
0.0000 \\
0.0000 \\
0.0000\end{array}$ & $\begin{array}{l}0.0000 \\
0.0000 \\
0.0000 \\
0.0000 \\
0.0000 \\
0.0000\end{array}$ & $\begin{array}{l}0.0000 \\
0.0000 \\
0.0000 \\
0.0000 \\
0.0000 \\
0.0200\end{array}$ & $\begin{array}{l}0.0100 \\
0.0500 \\
0.0000 \\
0.0000 \\
0.0000 \\
0.0000\end{array}$ \\
\hline $\begin{array}{l}R U-99 \\
R U-100 \\
R U-101 \\
R U-102 \\
R U-103 \\
R U-104 \\
R U-105\end{array}$ & $\begin{array}{l}0.0000 \\
0.0000 \\
0.0000 \\
0.0000 \\
0.0000 \\
0.0000 \\
0.0000\end{array}$ & $\begin{array}{l}0.0000 \\
0.0000 \\
0.0000 \\
0.0000 \\
0.0000 \\
0.0000 \\
0.0000\end{array}$ & $\begin{array}{l}0.0000 \\
0.0000 \\
0.0000 \\
0.0000 \\
0.0000 \\
0.0000 \\
0.0000\end{array}$ & $\begin{array}{l}0.0000 \\
0.0005 \\
0.0000 \\
0.0000 \\
0.0000 \\
0.0000 \\
0.0000\end{array}$ & $\begin{array}{l}0.0000 \\
\mathcal{C} .0000 \\
0.0000 \\
0.0000 \\
0.0000 \\
0.0500 \\
0.0000\end{array}$ & $\begin{array}{l}0.0000 \\
0.0 n 00 \\
0.0000 \\
0.0000 \\
0.0500 \\
0.0500 \\
0.0000\end{array}$ \\
\hline
\end{tabular}


NUCLIDE ACTIVITIES IN CURIES PER F.E. FOR RELOAD 9

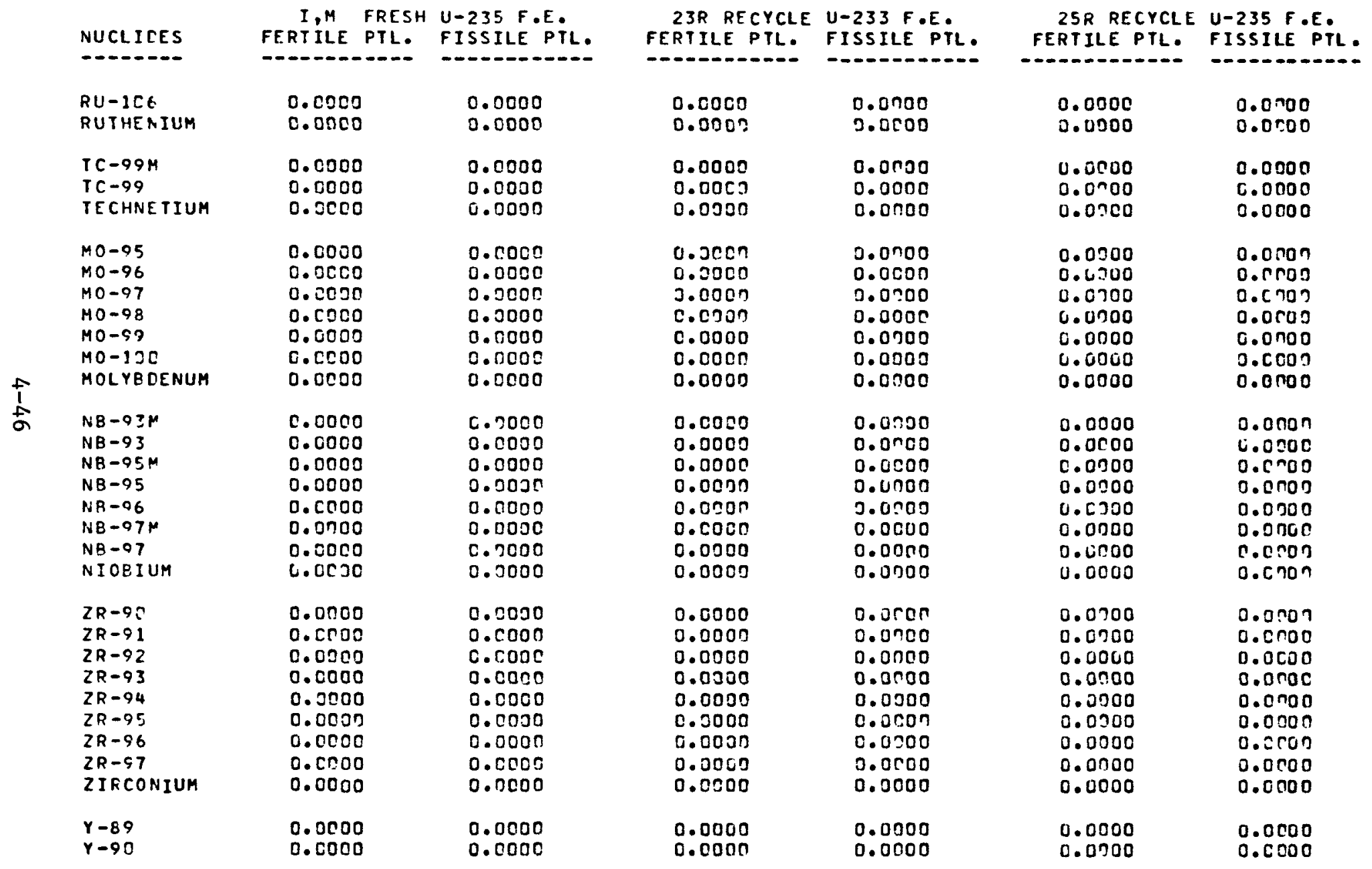


NUCLIDE ACTIVITIES IN CURIES PER F.E. FOR RELOAD 9

\begin{tabular}{|c|c|c|c|c|c|c|}
\hline NUCL IOES & $\begin{array}{l}\text { I.M FRESH } \\
\text { FERTILE PTL. }\end{array}$ & $\begin{array}{l}\text { U-235 F } \cdot E \text {. } \\
\text { FISSILE PTL. }\end{array}$ & $\begin{array}{l}\text { 23R RECYCLE } \\
\text { FERTILE PTL: }\end{array}$ & $\begin{array}{l}\text { U-233 F.E. } \\
\text { FISSILE PTL. }\end{array}$ & $\begin{array}{l}\text { 25R RECYCLE } \\
\text { FERTILE PTL: }\end{array}$ & $\begin{array}{l}\text { U-235 F.E. } \\
\text { FISSILE PTL. }\end{array}$ \\
\hline $\begin{array}{l}Y-91 M \\
Y-91 \\
Y-92 \\
Y-93 \\
Y T T E R B I U M\end{array}$ & $\begin{array}{l}0.0000 \\
0.0000 \\
0.0000 \\
0.0000 \\
0.0000\end{array}$ & $\begin{array}{l}0.0000 \\
0.0000 \\
0.0000 \\
0.0000 \\
0.0000\end{array}$ & $\begin{array}{l}0.0000 \\
0.0005 \\
0.0000 \\
0.0000 \\
0.0000\end{array}$ & $\begin{array}{l}0.0300 \\
0.0300 \\
0.0300 \\
0.0000 \\
0.0000\end{array}$ & $\begin{array}{l}0.0000 \\
0.0000 \\
0.0300 \\
0.0000 \\
0.0000\end{array}$ & $\begin{array}{l}0.0000 \\
0.0000 \\
0.0000 \\
0.0000 \\
0.0000\end{array}$ \\
\hline $\begin{array}{l}S R-86 \\
S R-87 \\
S R-88 \\
S R-89 \\
S R-90 \\
S R-91 \\
S T R O N T I U M\end{array}$ & $\begin{array}{l}0.0000 \\
0.0000 \\
0.0000 \\
0.0000 \\
0.0000 \\
0.0000 \\
0.0000\end{array}$ & $\begin{array}{l}0.0000 \\
0.0000 \\
0.0000 \\
0.0000 \\
0.300 n \\
0.0000 \\
0.0000\end{array}$ & $\begin{array}{l}0.000 n \\
0.0000 \\
0.0000 \\
0.0000 \\
0.0000 \\
0.0000 \\
0.0000\end{array}$ & $\begin{array}{l}0.0000 \\
0.0000 \\
0.0000 \\
0.0000 \\
0.0000 \\
0.0000 \\
0.0000\end{array}$ & $\begin{array}{l}4.0000 \\
0.0000 \\
0.0000 \\
0.0500 \\
0.0000 \\
0.0 n 60 \\
0.0000\end{array}$ & $\begin{array}{l}0.0000 \\
0.0 \operatorname{Qcos} \\
0.0000 \\
0.0000 \\
0.0000 \\
0.000 \\
0.0000\end{array}$ \\
\hline $\begin{array}{l}R B-85 \\
R B-86 \\
R B-87 \\
R \cup B I D I U M\end{array}$ & $\begin{array}{l}0.0000 \\
0.0000 \\
0.0000 \\
0.0000\end{array}$ & $\begin{array}{l}0.0000 \\
0.0000 \\
0.0000 \\
0.0000\end{array}$ & $\begin{array}{l}0.0000 \\
0.0000 \\
0.0000 \\
0.0000\end{array}$ & $\begin{array}{l}0.0300 \\
0.0300 \\
0.0000 \\
0.0000\end{array}$ & $\begin{array}{l}0.0000 \\
0.0000 \\
0.0000 \\
0.0000\end{array}$ & $\begin{array}{l}0.0000 \\
0.0700 \\
0.0000 \\
0.0000\end{array}$ \\
\hline $\begin{array}{l}K R-92 \\
K R-83 M \\
K R-83 \\
K R-84 \\
K R-85 M \\
K R-25 \\
K R-86 \\
K R Y P T O N\end{array}$ & $\begin{array}{l}0.0000 \\
0.0500 \\
0.0020 \\
0.0005 \\
0.0000 \\
0.0000 \\
0.0000 \\
0.0000\end{array}$ & $\begin{array}{l}0.0000 \\
0.0000 \\
0.0000 \\
0.0000 \\
0.0000 \\
0.0000 \\
0.0000 \\
0.0000\end{array}$ & $\begin{array}{l}0.0000 \\
0.0000 \\
0.0000 \\
0.0000 \\
0.0000 \\
0.0000 \\
0.0000 \\
0.0000\end{array}$ & $\begin{array}{l}0.0000 \\
0.0000 \\
0.0000 \\
0.0300 \\
0.0000 \\
0.0300 \\
0.0000 \\
0.0000\end{array}$ & $\begin{array}{l}0.0200 \\
0.0000 \\
0.0200 \\
0.0000 \\
0.0000 \\
0.0700 \\
0.0000 \\
0.0000\end{array}$ & $\begin{array}{l}0 . c n 00 \\
0.0000 \\
0.0000 \\
0.0000 \\
0.0000 \\
0.0000 \\
0.000 n \\
0.0000\end{array}$ \\
\hline $\begin{array}{l}B R-79 \\
B R-91 \\
2 R-82 \\
B R-83 \\
B R O N I N E\end{array}$ & $\begin{array}{l}0.0000 \\
0.0000 \\
0.0000 \\
0.0000 \\
0.0000\end{array}$ & $\begin{array}{l}0.0000 \\
0.0000 \\
0.0000 \\
0.0000 \\
0.0000\end{array}$ & $\begin{array}{l}0.0000 \\
0.0000 \\
0.0000 \\
0.0000 \\
0.0000\end{array}$ & $\begin{array}{l}0.0000 \\
0.0000 \\
0.0200 \\
0.0900 \\
0.0000\end{array}$ & $\begin{array}{l}0.0000 \\
0.0000 \\
0.0000 \\
0.0300 \\
0.0300\end{array}$ & $\begin{array}{l}0.0000 \\
0.0000 \\
0.0000 \\
0.0000 \\
0.0000\end{array}$ \\
\hline $\begin{array}{l}S E-79 \\
S E-80\end{array}$ & $\begin{array}{l}0.0000 \\
0.0000\end{array}$ & $\begin{array}{l}0.0000 \\
0.0000\end{array}$ & $\begin{array}{l}0.0000 \\
0.0000\end{array}$ & $\begin{array}{l}0.0000 \\
0.0000\end{array}$ & $\begin{array}{l}0.0000 \\
0.0000\end{array}$ & $\begin{array}{l}c .0000 \\
0.0000\end{array}$ \\
\hline
\end{tabular}


NUCLIDE ACTIVITIES IN CURIES PER F.E. FOR RELOAD 9

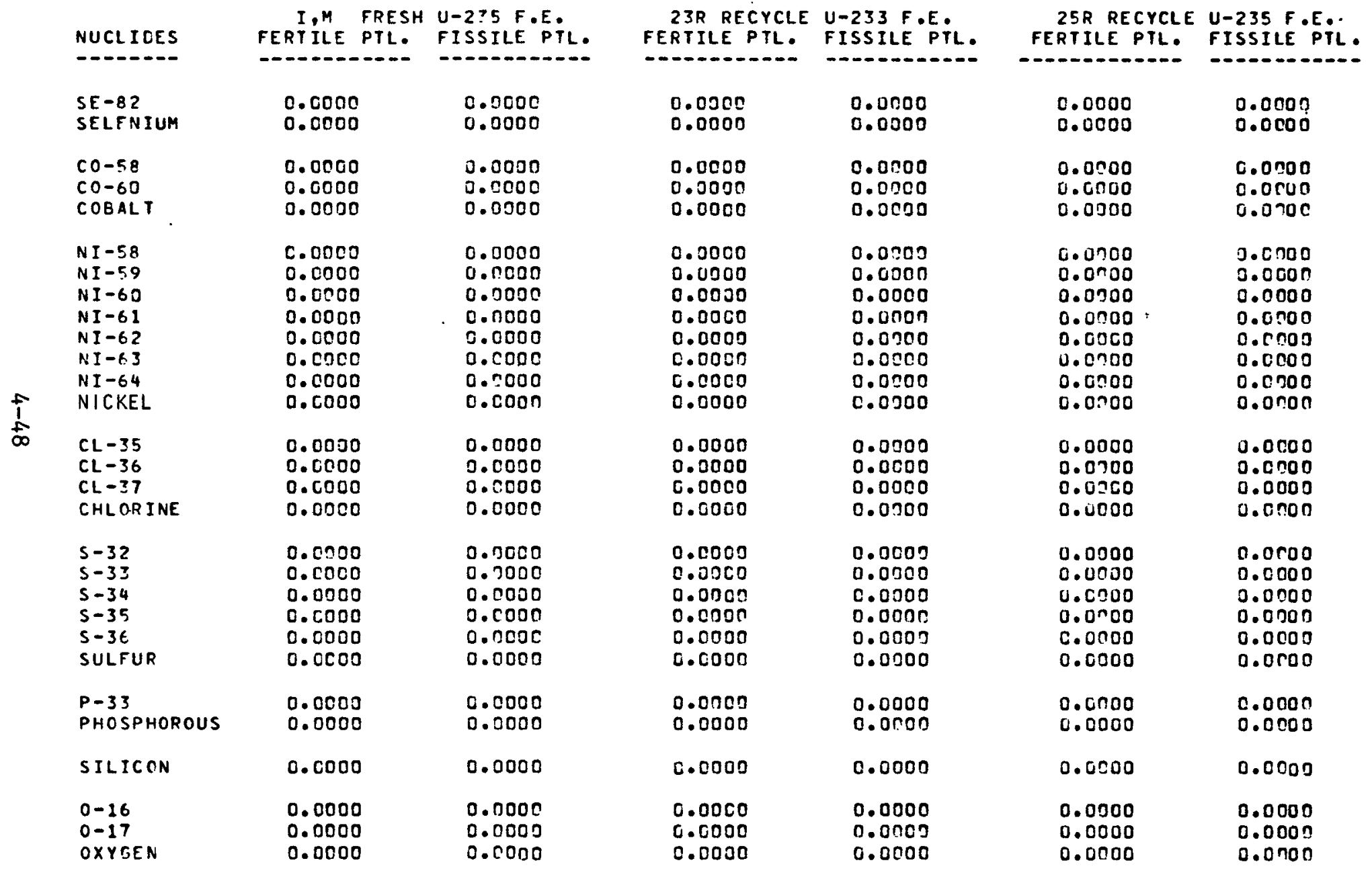


NUCLIDE ACTIVITIES IN CURIES PER F.E. FOR RELOAD 9

\begin{tabular}{|c|c|c|c|c|c|c|}
\hline NUCLICES & $\begin{array}{l}\text { I,M FRESH } \\
\text { FERTILE PTL. }\end{array}$ & $\begin{array}{l}\text { U-235 F.E. } \\
\text { FISSILE PTL. }\end{array}$ & $\begin{array}{l}\text { 23R RECYCLE } \\
\text { FERTILE PTL. }\end{array}$ & $\begin{array}{l}\text { U-233 F.E. } \\
\text { FISS.ILE PTL. }\end{array}$ & $\begin{array}{l}\text { 25R RECYCLE } \\
\text { FERTILE PTL. }\end{array}$ & $\begin{array}{l}\text { U-235 F EE. } \\
\text { FISSILE PTL. }\end{array}$ \\
\hline $\begin{array}{l}N-14 \\
N-15 \\
N I T P O G E N\end{array}$ & $\begin{array}{l}c .0000 \\
0.0000 \\
0.0000\end{array}$ & $\begin{array}{l}0.3000 \\
0.0000 \\
0.0000\end{array}$ & $\begin{array}{l}0.0000 \\
0.0000 \\
0.0000\end{array}$ & $\begin{array}{l}0.0 \cap 50 \\
0.0000 \\
0.0000\end{array}$ & $\begin{array}{l}0.0700 \\
0.0300 \\
0.0500\end{array}$ & 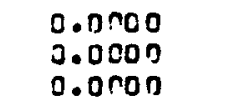 \\
\hline $\begin{array}{l}C-12 \\
C-13 \\
C-14 \text { FROM } C \\
C-14 \text { FROM N } \\
C-14 \text { FROM } 0 \\
\text { CARPON }\end{array}$ & $\begin{array}{l}0.0000 \\
0.0000 \\
0.0000 \\
0.4000 \\
0.0000 \\
0.0000\end{array}$ & $\begin{array}{l}0.0000 \\
0.0000 \\
0.0000 \\
0.0000 \\
0.0000 \\
0.0000\end{array}$ & $\begin{array}{l}0.0000 \\
0.0000 \\
0.0000 \\
0.0000 \\
0.0000 \\
0.0900\end{array}$ & $\begin{array}{l}0.0 \cap 00 \\
0.0000 \\
0.0 n 00 \\
0.0000 \\
0.0000 \\
0.0000\end{array}$ & $\begin{array}{l}0.0000 \\
0.0000 \\
0.0000 \\
0.0000 \\
0.0000 \\
0.0000\end{array}$ & $\begin{array}{l}0.0000 \\
0.0500 \\
0.0700 \\
0.0700 \\
0.0000 \\
0.0000\end{array}$ \\
\hline $\begin{array}{l}B-10 \\
B-11 \\
\text { BORON }\end{array}$ & $\begin{array}{l}0.0000 \\
0.0000 \\
0.0000\end{array}$ & $\begin{array}{l}0.0000 \\
0.0000 \\
0.0000\end{array}$ & $\begin{array}{l}0.0030 \\
0.0000 \\
0.0000\end{array}$ & $\begin{array}{l}0.0000 \\
0.0000 \\
0.0000\end{array}$ & $\begin{array}{l}0.0000 \\
0.0700 \\
0.0000\end{array}$ & $\begin{array}{l}0.0 n 00 \\
0.0000 \\
0.0 n 00\end{array}$ \\
\hline $\begin{array}{l}\text { LI }-6 \\
\text { LI }-7 \\
\text { LITHIUM }\end{array}$ & $\begin{array}{l}0.0000 \\
0.0000 \\
0.0000\end{array}$ & $\begin{array}{l}0.0000 \\
0.0000 \\
0.0000\end{array}$ & $\begin{array}{l}0.0000 \\
0.0005 \\
0.0050\end{array}$ & $\begin{array}{l}0.0000 \\
0.0090 \\
0.0000\end{array}$ & $\begin{array}{l}0.0 \cap 00 \\
C .0000 \\
0.0000\end{array}$ & $\begin{array}{l}0.050 n \\
0.0 n g 0 \\
0.0000\end{array}$ \\
\hline $\begin{array}{l}\text { H-3 FFOM LIG } \\
\text { H-3 FPOM BIS } \\
\text { H-3 F. YIELD } \\
\text { TRITIUM }\end{array}$ & $\begin{array}{l}0.0000 \\
0.0000 \\
0.0000 \\
0.0000\end{array}$ & $\begin{array}{l}0.000 n \\
0.000 \mathrm{C} \\
0.0000 \\
0.0000\end{array}$ & $\begin{array}{l}0.0000 \\
0.0000 \\
0.0000 \\
0.0000\end{array}$ & $\begin{array}{l}0.0 n 00 \\
0.0 n 00 \\
0.0000 \\
0.0000\end{array}$ & $\begin{array}{l}0.0000 \\
0.0000 \\
0.0500 \\
0.0000\end{array}$ & $\begin{array}{l}0.0000 \\
0.0500 \\
0.000 n \\
0.0 \operatorname{con}\end{array}$ \\
\hline MUD & $0.0 n 00$ & 0.0000 & 0.0000 & 0.0000 & 0.0000 & $0.0 \cos \theta$ \\
\hline
\end{tabular}




\section{TABLE 5}

RELOAD 13 - FUEL ELEMENT COMPOSITION AS DISCHARGED

The MS, 23RS, and 25RS elements discharged at reload 13 were in the reactor core for $4 \mathrm{yr}$. Reload 13 spent fuel elements are typical for an HTGR operating at equilibrium with recycle fuel. 
NUCLIDE WEIGHTS IN GRAMS PER F.E. AT TIME 180.0 DAYS AFTER DISCHARGE

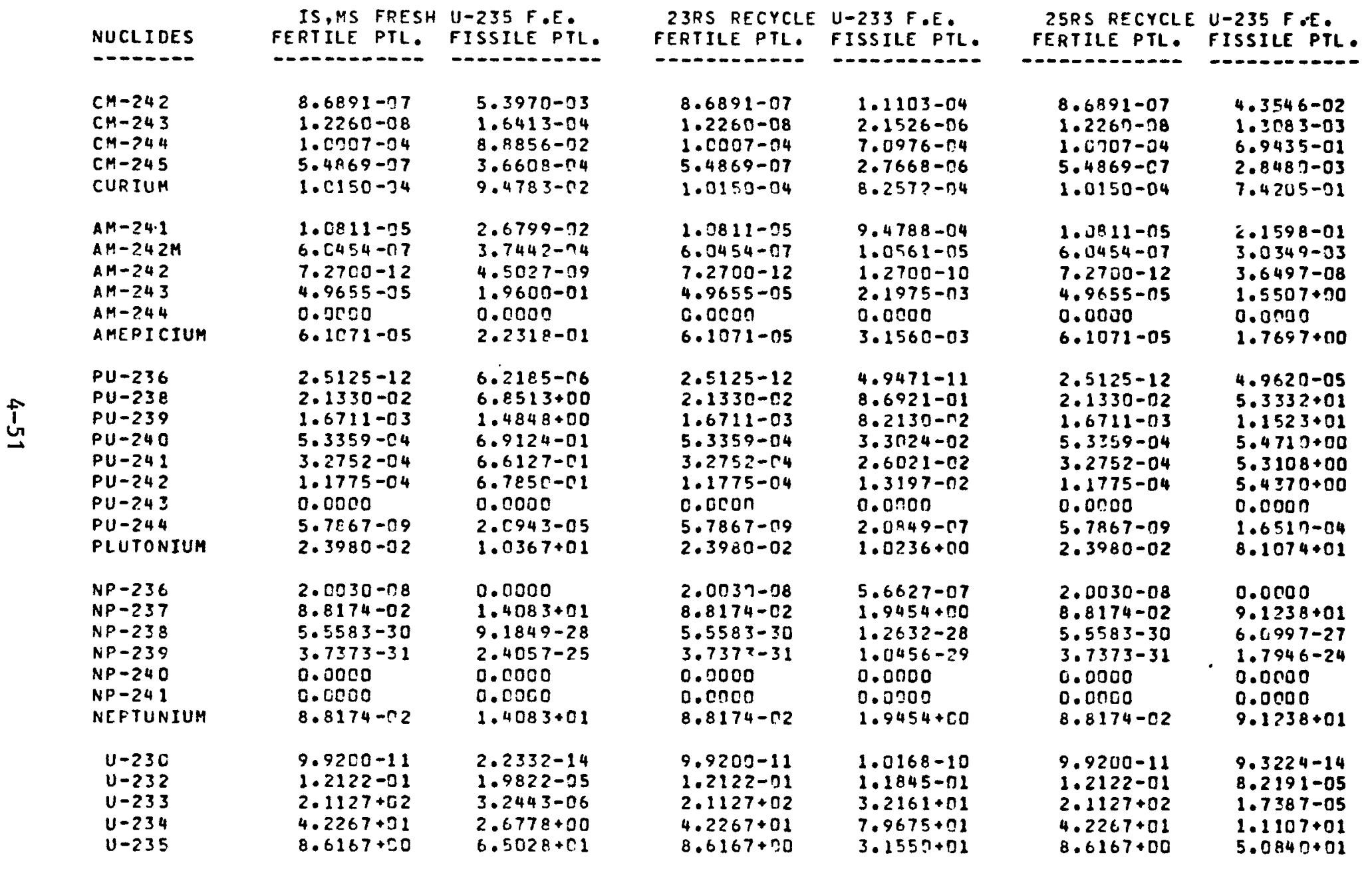


NUCLIOE WEIGHTS IN GRAMS PER F.E. AT TIME 180.0 DAYS AFTER DISCHARGE

\begin{tabular}{|c|c|c|c|c|c|c|}
\hline NUCLIDES & $\begin{array}{l}\text { IS,MS FRESH } \\
\text { FERTILE PTL. }\end{array}$ & $\begin{array}{l}\text { U-235 F.E. } \\
\text { FISSILE PTL. }\end{array}$ & $\begin{array}{l}\text { 23RS RECYCLE } \\
\text { FERTILE PTL. }\end{array}$ & $\begin{array}{l}\text { U-233 F.E. } \\
\text { FISSILE PTL. }\end{array}$ & $\begin{array}{l}\text { 25RS RECYCLE } \\
\text { FERTILE PTL. }\end{array}$ & $\begin{array}{l}\text { U-235 F.E. } \\
\text { FISSILE PTL. }\end{array}$ \\
\hline $\begin{array}{l}U-236 \\
U-237 \\
U-238 \\
U-239 \\
U-240 \\
\text { URANIUM }\end{array}$ & $\begin{array}{l}1.3594+00 \\
3.4910-11 \\
5.9095-05 \\
0.0000 \\
0.0030 \\
2.6364+02\end{array}$ & $\begin{array}{l}1.1510+02 \\
2.7008-09 \\
3.7535+01 \\
0.0000 \\
0.0000 \\
2.2034+02\end{array}$ & $\begin{array}{l}1.3594+00 \\
3.4917-11 \\
5.9095-05 \\
0.0000 \\
0.0001 \\
2.6364+02\end{array}$ & $\begin{array}{l}1.6082+01 \\
4.2082-10 \\
1.6400-03 \\
0.0000 \\
0.0000 \\
1.5959+02\end{array}$ & $\begin{array}{l}1.3594+00 \\
3.4910-11 \\
5.9595-05 \\
0.0000 \\
0.0900 \\
2.6364+02\end{array}$ & $\begin{array}{l}1.0542+03 \\
2.3602-08 \\
2.8000+02 \\
0.0 \times 00 \\
0.0000 \\
1.3962+03\end{array}$ \\
\hline $\begin{array}{l}P A-230 \\
P A-231 \\
P A-232 \\
P A-233 \\
P A-234 M \\
P A-234 \\
P A-235 \\
\text { PROTACTINIUM }\end{array}$ & $\begin{array}{l}6.2596-12 \\
5.8657-02 \\
0.0000 \\
1.9835-01 \\
7.7898-10 \\
2.6850-07 \\
0.0000 \\
2.5701-01\end{array}$ & $\begin{array}{l}9.7219-16 \\
9.7975-16 \\
0.0000 \\
2.6166-10 \\
0.0000 \\
6.3581-11 \\
0.0000 \\
9.7978-06\end{array}$ & $\begin{array}{l}6.2596-12 \\
5.8657-02 \\
0.0000 \\
1.9835-01 \\
7.7898-10 \\
2.6850-07 \\
0.0700 \\
2.5751-01\end{array}$ & $\begin{array}{l}6.4231-13 \\
6.4764-03 \\
0.0000 \\
2.0679-09 \\
0.0000 \\
1.7932-15 \\
0.0000 \\
6.4764-03\end{array}$ & $\begin{array}{l}6.2596-12 \\
5.8657-02 \\
0.0000 \\
1.9835-01 \\
7.7898-10 \\
2.6950-07 \\
0.0000 \\
2.5701-01\end{array}$ & $\begin{array}{l}4.0586-15 \\
4.0 \times 06-05 \\
0.0000 \\
5.2394-09 \\
0.0000 \\
4.7435-10 \\
0.0000 \\
4.0812-05\end{array}$ \\
\hline $\begin{array}{l}T H-226 \\
T H-227 \\
T H-228 \\
T H-229 \\
T H-23 C \\
T H-231 \\
T H-232 \\
T H-233 \\
T H-234 \\
T H-235 \\
\text { THORIUM }\end{array}$ & $\begin{array}{l}1.0067-13 \\
7.6085-09 \\
1.3769-73 \\
1.3659-03 \\
3.1050-01 \\
3.5025-11 \\
7.9359+03 \\
0.0000 \\
1.2256-05 \\
0.0000 \\
7.9362+03\end{array}$ & $\begin{array}{l}2.2663-17 \\
6.2446-13 \\
2.2377-07 \\
3.036 C-08 \\
3.8556-05 \\
2.6432-10 \\
9.910 C-06 \\
0.000 c \\
5.4479-10 \\
0.0000 \\
4.8721-05\end{array}$ & $\begin{array}{l}1.0067-13 \\
7.6085-09 \\
1.3769-03 \\
1.3659-03 \\
3.1050-01 \\
3.5025-11 \\
7.9359+03 \\
0.0000 \\
1.2256-05 \\
0.0000 \\
7.9362+03\end{array}$ & $\begin{array}{l}0.0000 \\
3.8099-10 \\
4.4931-03 \\
5.8919-03 \\
2.4274-02 \\
1.2824-10 \\
8.6102-05 \\
0.0000 \\
1.5214-13 \\
0.0000 \\
3.4747-02\end{array}$ & $\begin{array}{l}1.0067-13 \\
7.6085-09 \\
1.3769-03 \\
1.3659-03 \\
3.1050-01 \\
3.5025-11 \\
7.9359+03 \\
0.0000 \\
1.2256-05 \\
0.0000 \\
7.9362+03\end{array}$ & $\begin{array}{l}9.4604-17 \\
2.5990-12 \\
9.2536-07 \\
1.2497-07 \\
1.6189-04 \\
2.0666-10 \\
1.7672-04 \\
0.0000 \\
4.0642-09 \\
0.0000 \\
3.3967-04\end{array}$ \\
\hline $\begin{array}{l}A C-225 \\
A C-227 \\
A C-228 \\
A C-229 \\
A C T I N I U M\end{array}$ & $\begin{array}{l}4.6688-09 \\
3.3385-0.6 \\
1.6889-09 \\
0.0000 \\
3.3448-06\end{array}$ & $\begin{array}{l}1.0377-13 \\
2.8488-10 \\
1.7461-18 \\
0.0000 \\
2.8488-10\end{array}$ & $\begin{array}{l}4.6688-09 \\
3.3385-06 \\
1.6889-09 \\
0.0309 \\
3.3448-06\end{array}$ & $\begin{array}{l}2.0139-0.8 \\
1.7636-07 \\
6.0680-16 \\
0.0900 \\
1.7636-07\end{array}$ & $\begin{array}{l}4.6688-09 \\
3.3385-06 \\
1.6889-09 \\
0.0000 \\
3.3448-06\end{array}$ & $\begin{array}{l}4.2716-13 \\
1.1858-09 \\
9.2345-18 \\
0.0000 \\
1.1858-09\end{array}$ \\
\hline $\begin{array}{l}R A-222 \\
R A-223\end{array}$ & $\begin{array}{l}2.0267-15 \\
4.6978-10\end{array}$ & $\begin{array}{l}4.5622-19 \\
3.8557-14\end{array}$ & $\begin{array}{l}2.0267-15 \\
4.6978-10\end{array}$ & $\begin{array}{l}2.0774-15 \\
2.3524-11\end{array}$ & $\begin{array}{l}2.0267-15 \\
4.6978-10\end{array}$ & $\begin{array}{l}1.9045-18 \\
1.6043-13\end{array}$ \\
\hline
\end{tabular}


NUCLIDE NEIGHTS IN GRAMS PER F.E. AT TIME 180.0 DAYS AFTER DISCHARGE

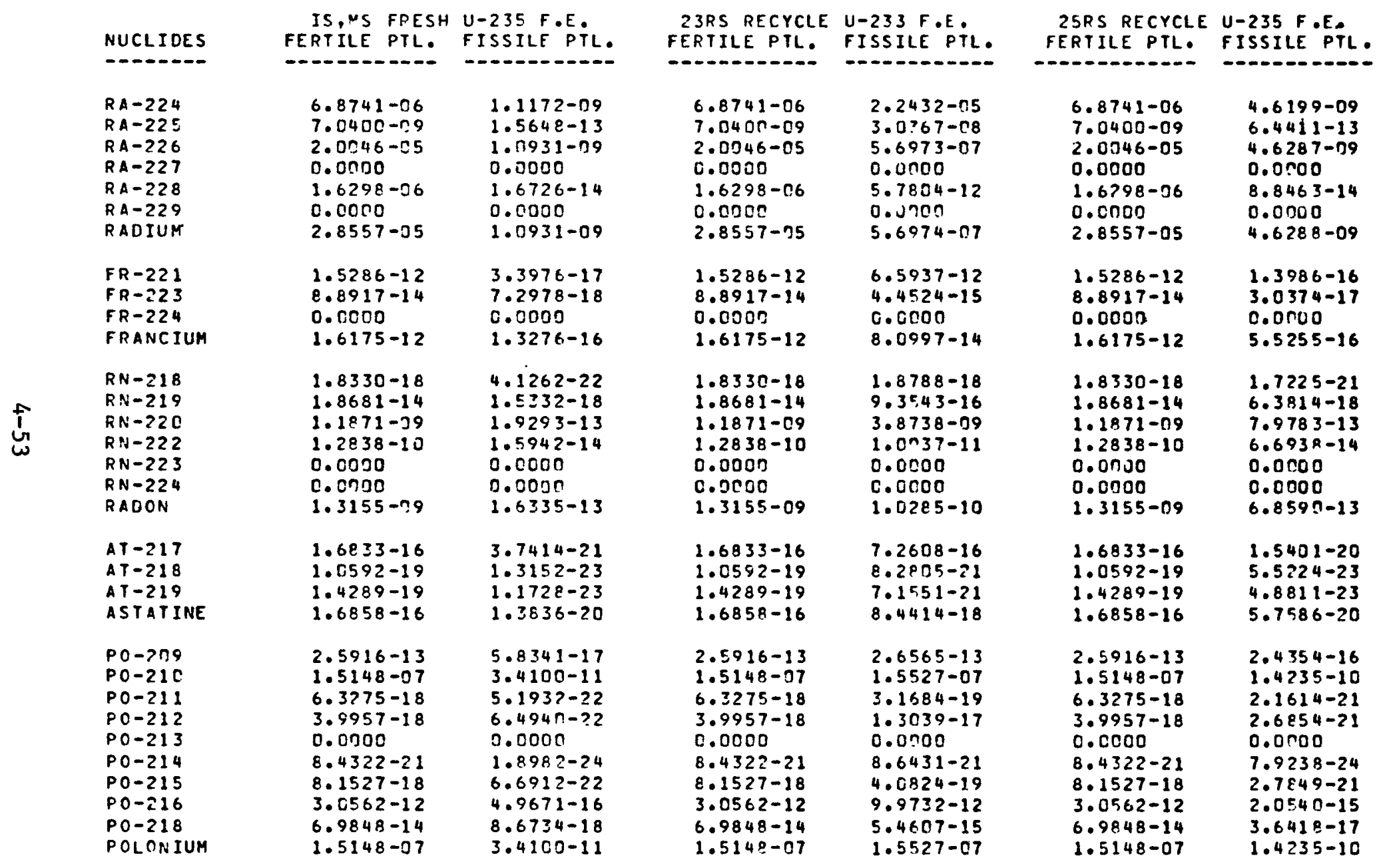


NUCLIDE WEIGHTS IN GRAMS PER F.E. AT TIME 180.0 DAYS AFTER DISCHARGE

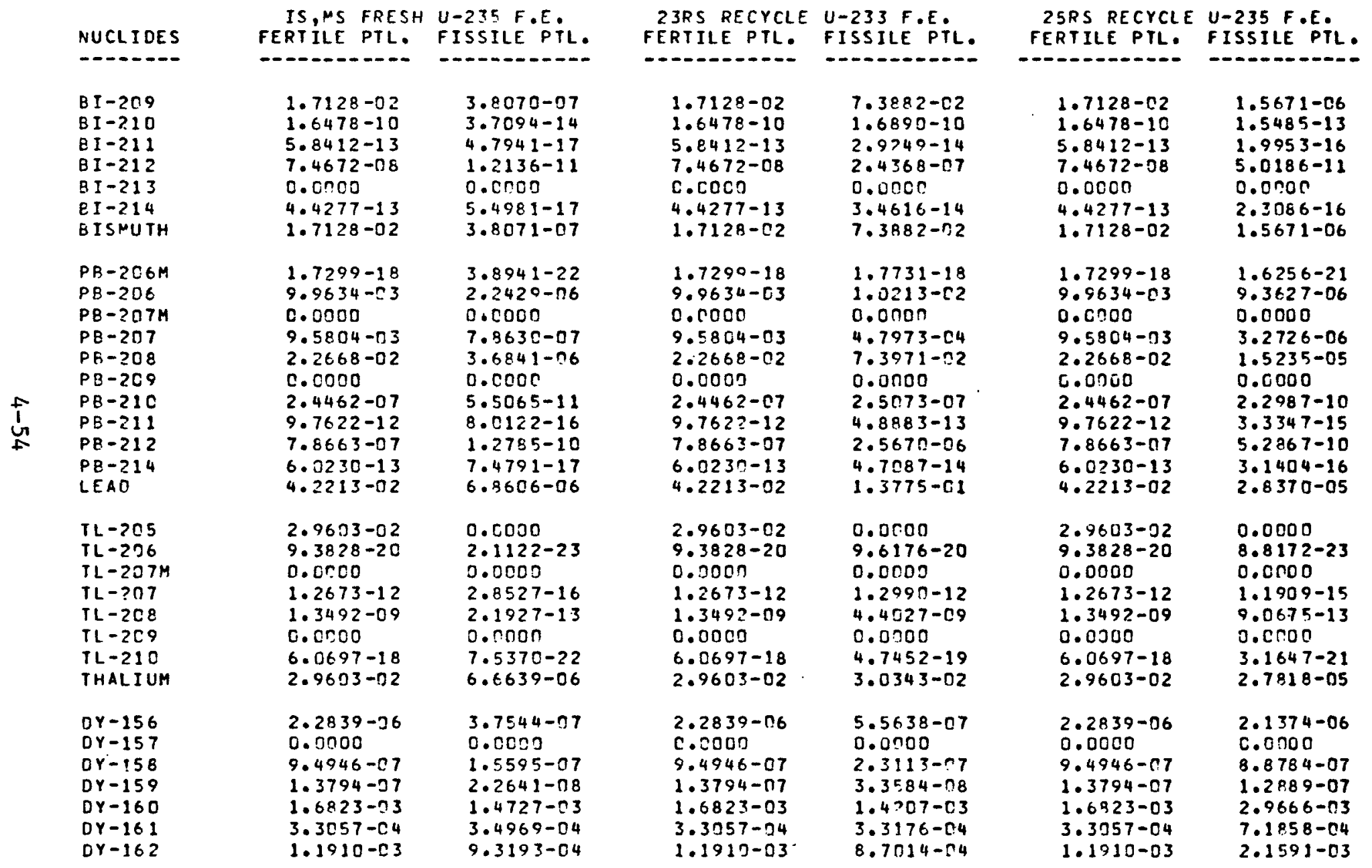


NUCL IDE WEIGHTS IN GRAMS PEP F.E. AT TIME 183.0 OAYS AFTER DISCHARGE

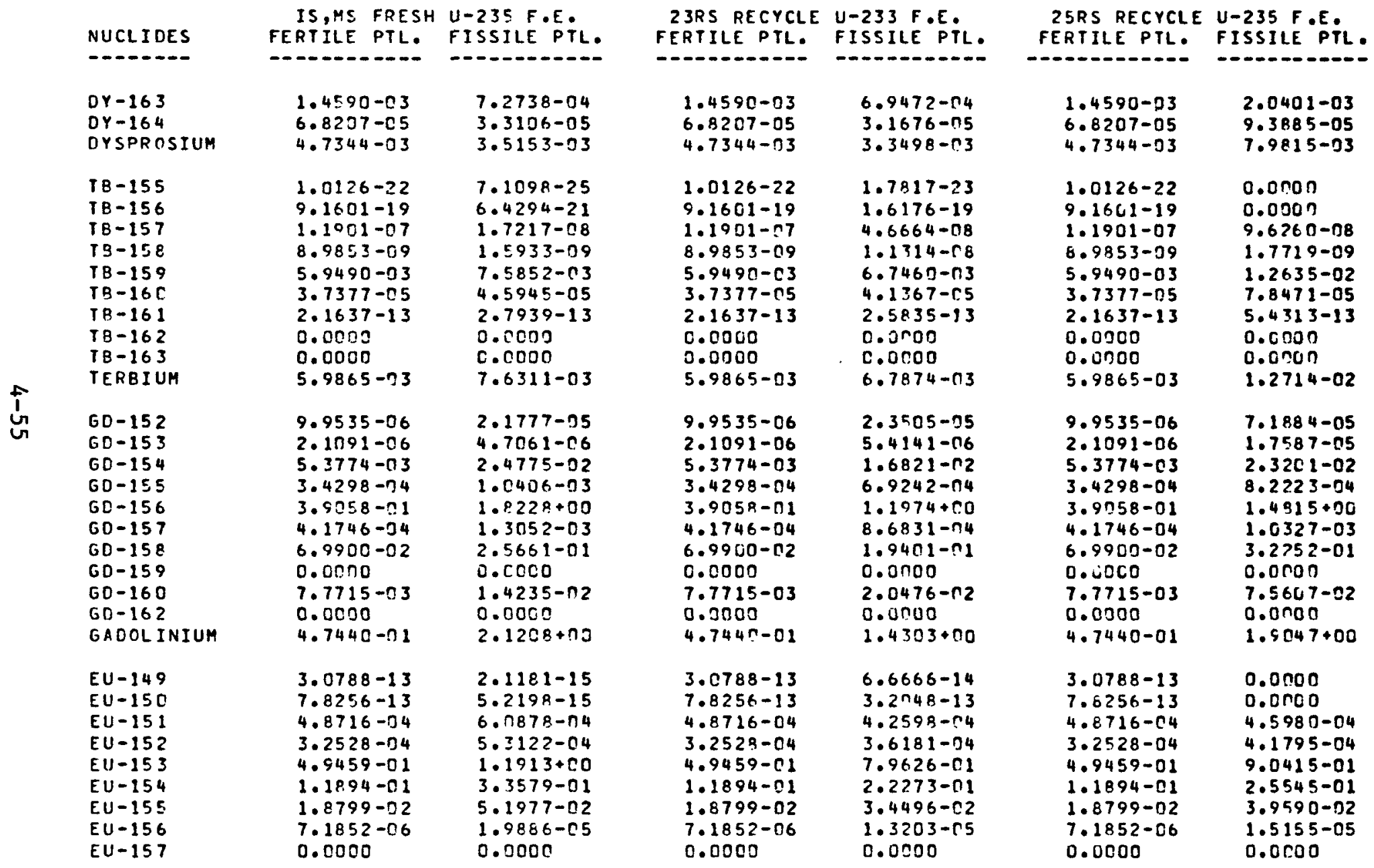


NUCLIDE WEIGHTS IN GRAMS PER F.E. AT TIME $180 . C$ DAYS AFTER DISCHARGE

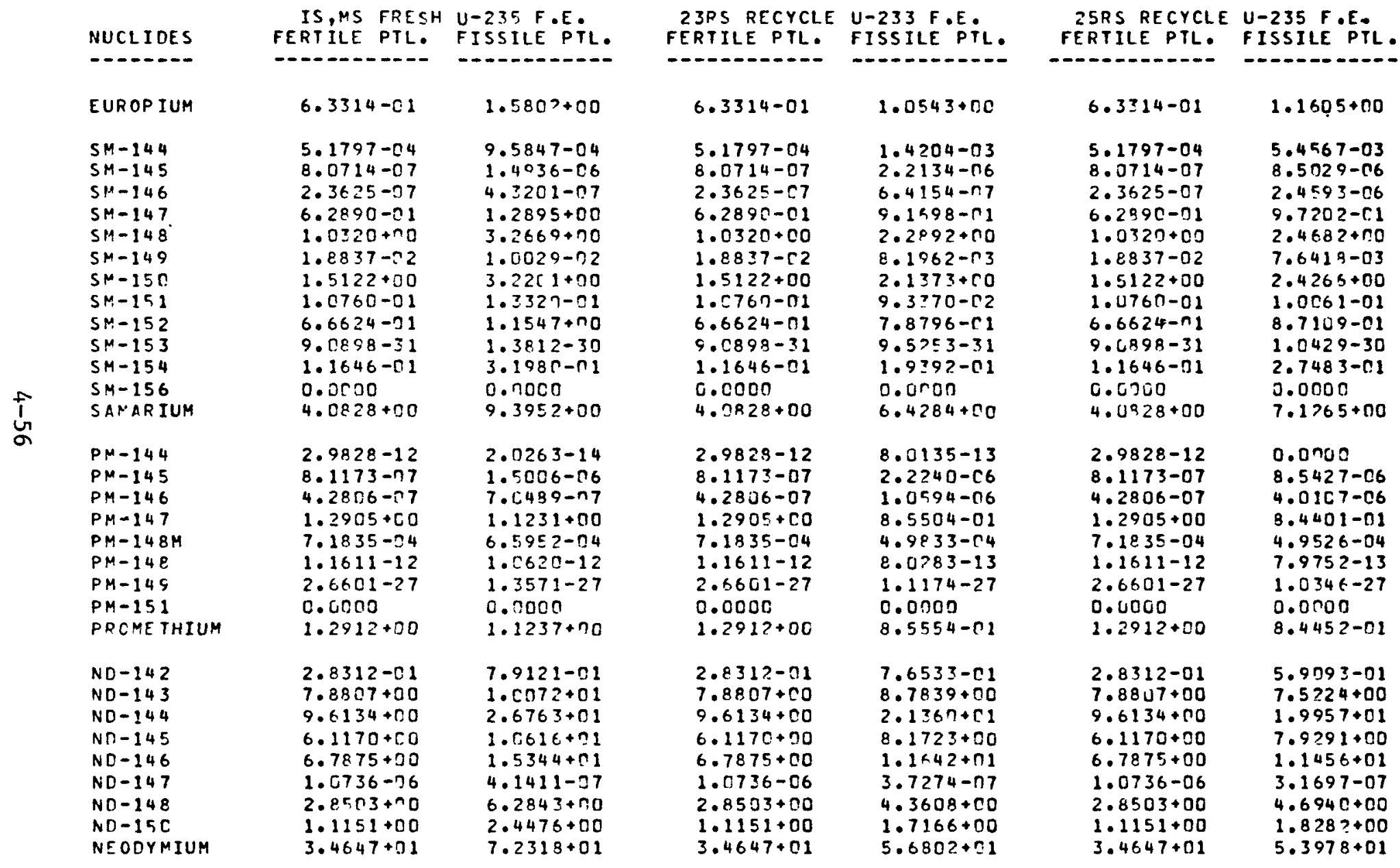


NUCLIDE WEIGHTS IN GRAMS PER F.E. AT TIME 180.C DAYS AFTER DISCHARgE

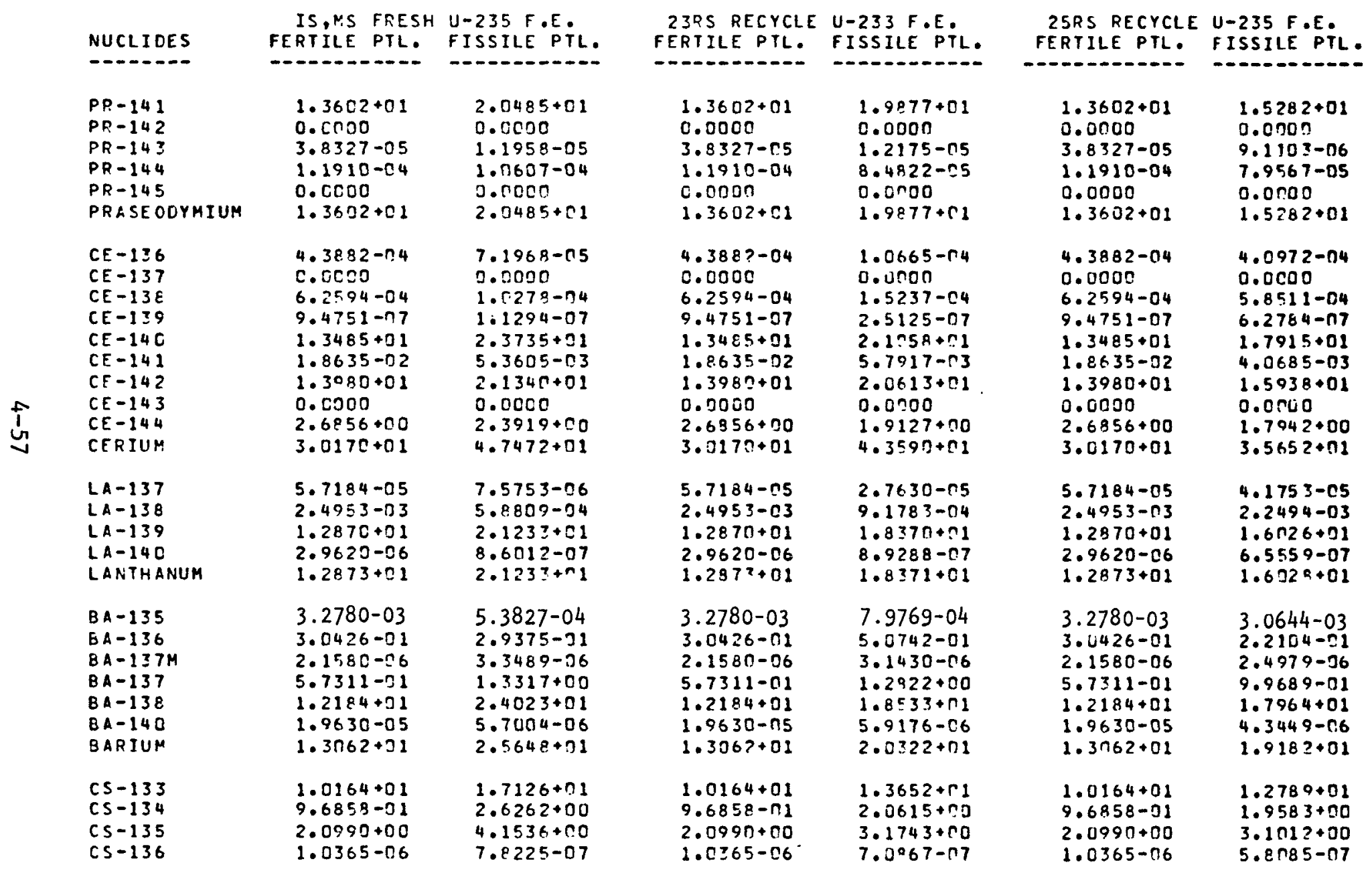


NUCLIDE WEIGHTS IN GRAMS PER F.E. AT TIME 180.0 DAYS AFTER DISCHARGE

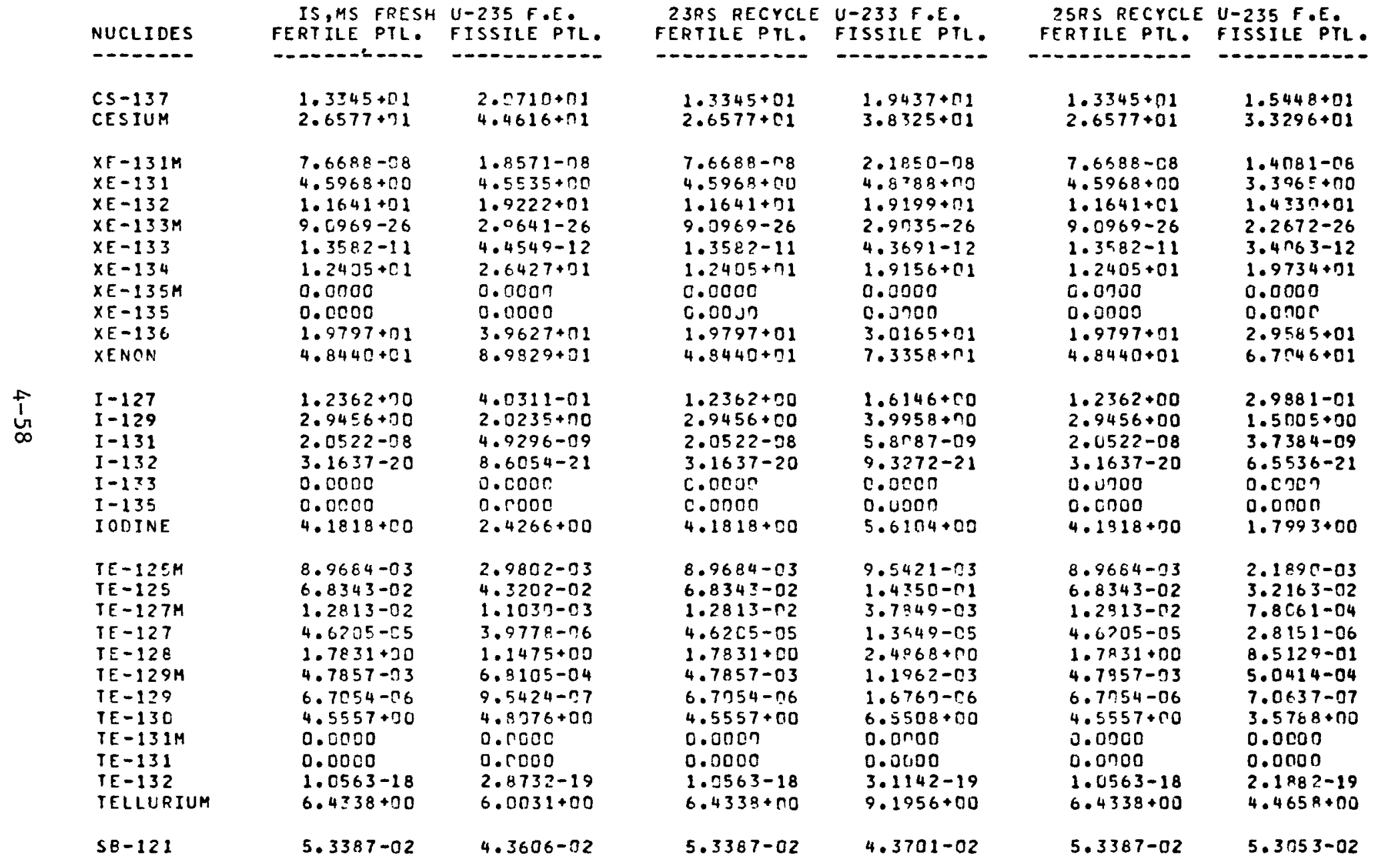


NUCLIOE WEIGHTS IN GRAMS PER F.E. AT TIME 180.0 DAYS AFTER DISCHARGE

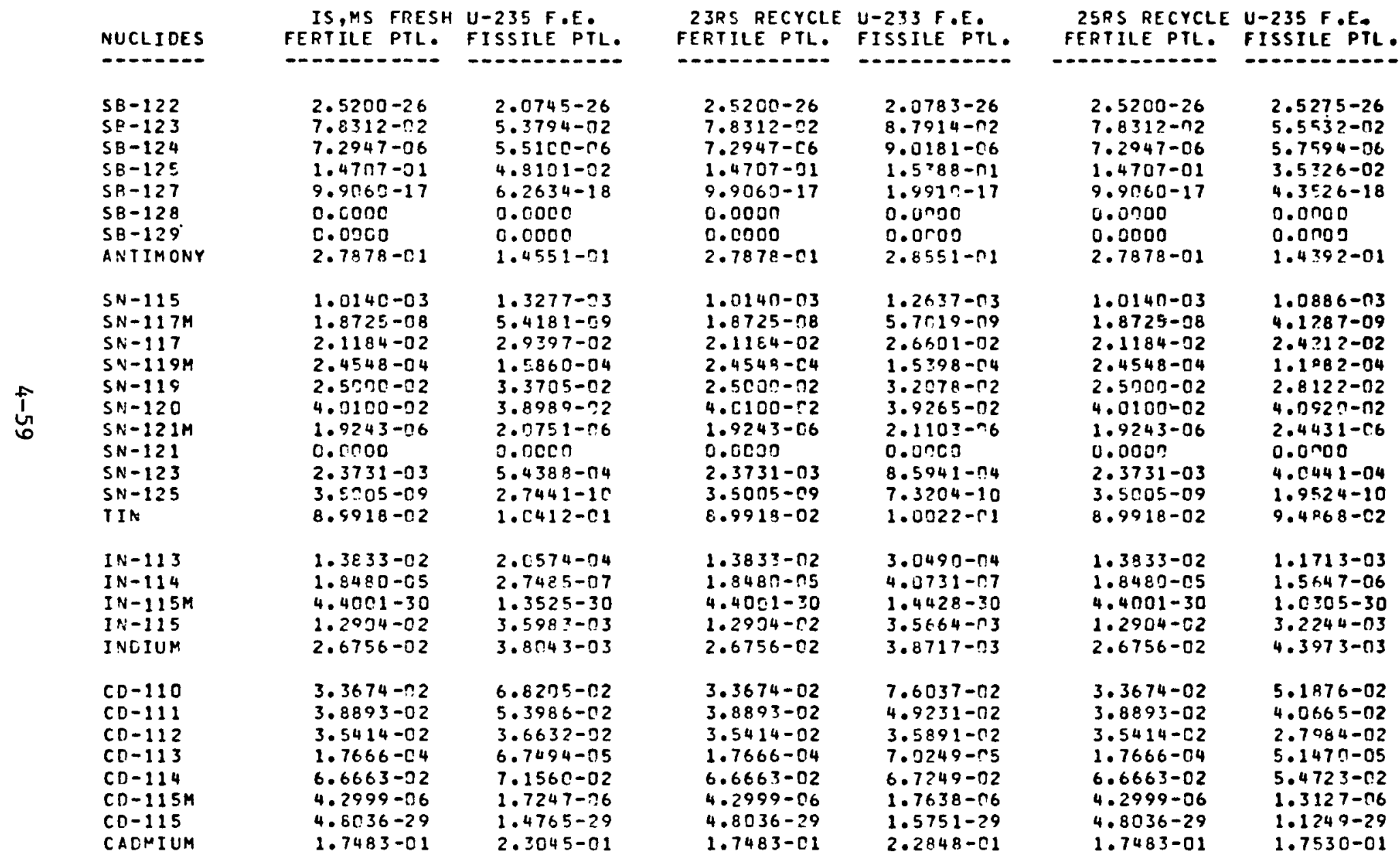


NUCLIDE WEIGHTS IN GRAMS PER F.E. AT TIME 180.0 DAYS AFTER DISCHARGE

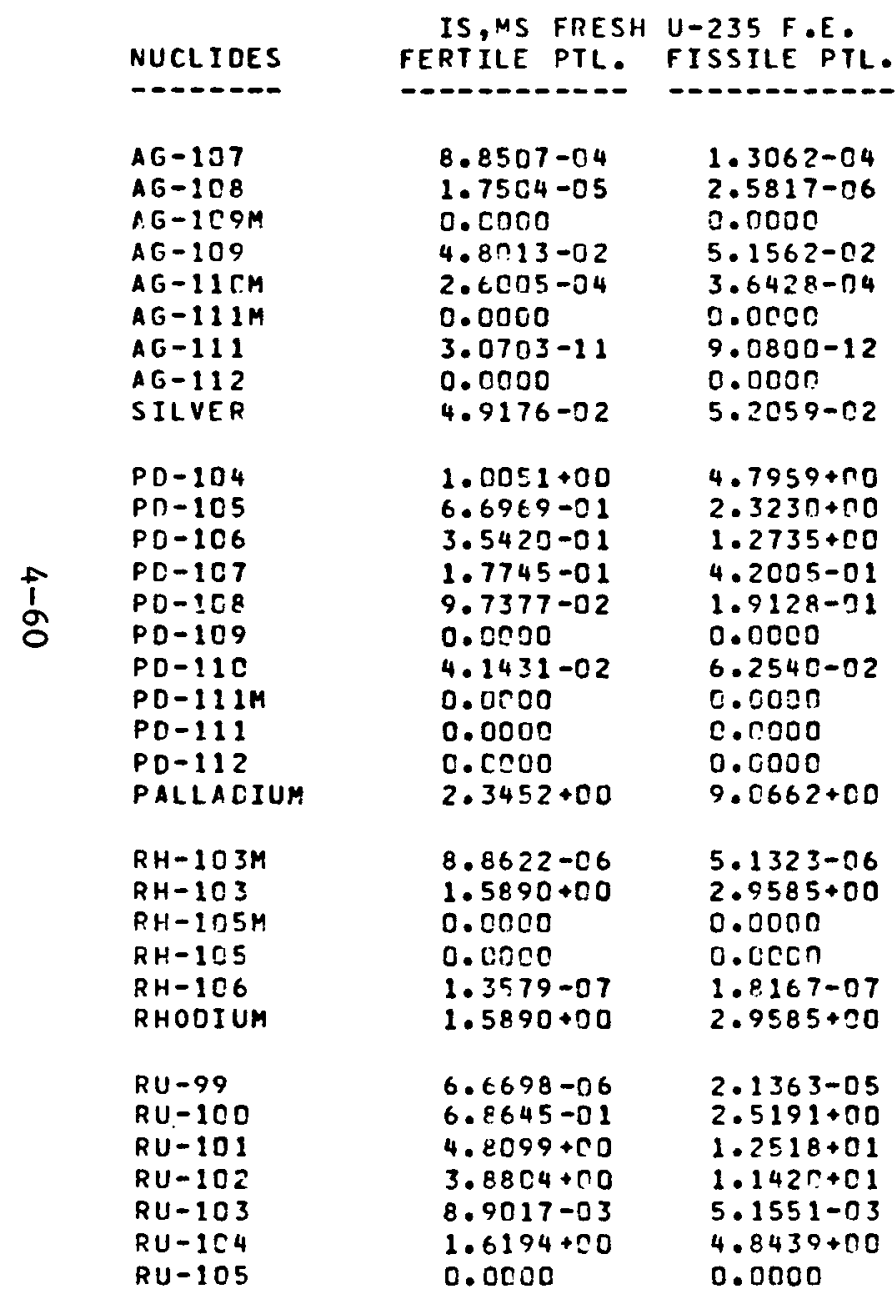

23RS RECYCLE U-233 F.E. FERTILE PTL. FISSILE PTL.

2SRS RECYCLE U-235 F.E. FERTILE PTL. FISSILE PTL.

\section{$8.85 C 7-\mathrm{Cu}_{4}$}

$1.7504-7.5$

0.0000

$4.8013-02$

$2.6005-04$

C. 0000

$3.0703-11$

0.0000

$4.9176-c 2$

$1.0051+00$

6.6969-01

$3.542 n-01$

$1.7745-01$

$9.7377-02$

0.0000

4.1431-02

0.0000

0.0000

C.000

$2.3452+90$

$8.8622-06$

$1.5890+00$

c. 0000

0.0000

1. 3579-.

$1.5890+00$

$6.6698-06$

$6.8645-01$

$4.8099+C 0$

$3.8804+C 0$

$8.9017-03$

$1.6194+00$

0.0000

\section{$1.9348-04$}

$3.8255-.06$

C.0000

$5.2910-02$

$3.8681-C 4$

0.0000

$9.6420-12$

0.0000

$5.3394-22$

$2.4523+00$

$1.000 \mathrm{C}+\mathrm{nO}$

$7.1299-01$

2.7?71-ก1

$1.4482-01$

0.0050

6.1365-02

0.0 ก00

0.0000

$0.0 \mathrm{con}$

$4.7342+100$

3. $3 \cos -1.6$

$1.6468+00$

0.0 ODC

$0 . u n c 0$

1. $1.10-07$

$1.6468+00$

$1.5397-05$

1. $8103+n 0$

$7.5277+50$

$6.3778+r 0$

$3.8175-03$

$2.6260+00$

0.0500

$\begin{array}{ll}8.8507-04 & 7.4307-04 \\ 1.7504-05 & 1.4695-05 \\ 0.0000 & 0.0000 \\ 4.8013-02 & 3.8694-02 \\ 2.6005-04 & 2.7357-04 \\ 0.0000 & 0.0000 \\ 3.0703-11 & 6.9149-12 \\ 0.0700 & 0.0000 \\ 4.4176-02 & 3.9725-02 \\ & \\ 1.0051+00 & 3.4963+00 \\ 6.6969-01 & 1.7381+00 \\ 3.5420-01 & 9.5187-01 \\ 1.7745-01 & 3.1384-01 \\ 9.7377-02 & 1.4346-01 \\ 0.0000 & 0.0000 \\ 4.1431-02 & 4.6636-02 \\ 0.0000 & 0.0000 \\ 0.0000 & 0.0000 \\ 0.0000 & 0.0000 \\ 2.3452+00 & 6.6902+00 \\ 8.8622-06 & 3.8430-06 \\ 1.5890+00 & 2.1658+00 \\ 0.0000 & 0.0000 \\ 0.0700 & 0.0000 \\ 1.3579-07 & 1.3628-07 \\ 1.5890+00 & 2.1658+00 \\ & \\ 6.6598-06 & 1.5567-05 \\ 6.8645-01 & 1.8353+00 \\ 4.8099+00 & 9.1366+00 \\ 3.8804+00 & 8.3357+00 \\ 8.9717-03 & 3.8601-03 \\ 1.6194+00 & 3.5363+00 \\ 0.0000 & 0.0000 \\ & \end{array}$


THIS SEGMENT WAS OISCHARGED AT THE BEGINNING OF RELOAD 13 AND WAS ORIGINALY LOADED AT RELOAD 9

NUCLIDE WEIGHTS IN GRAMS PER F.E. AT TIME 180.0 DAYS AFTER DISCHARGE

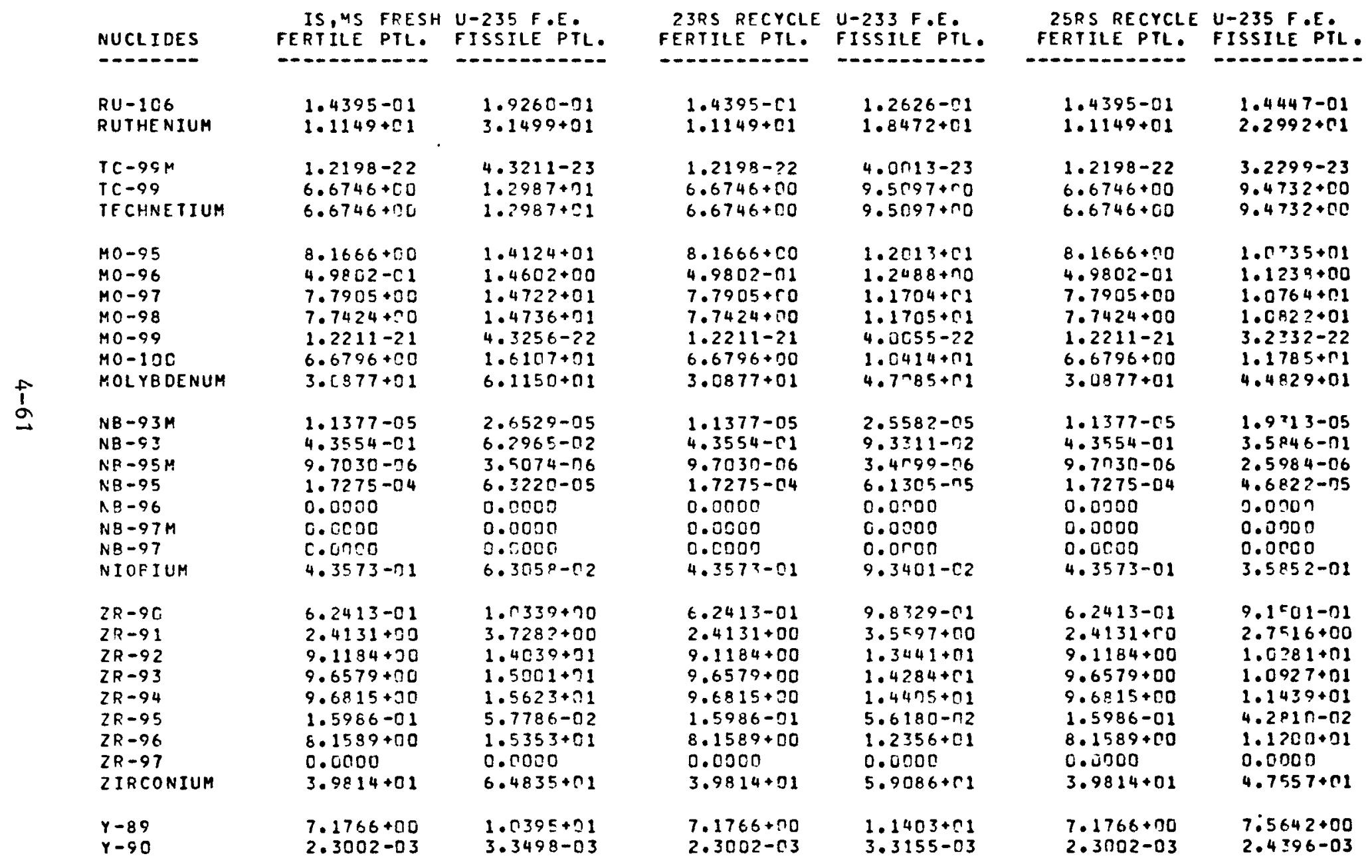


NUCLIDE WEIGHTS IN GRAMS PER F.E. AT TIME 180.0 DAYS AFTER DISCHARGE

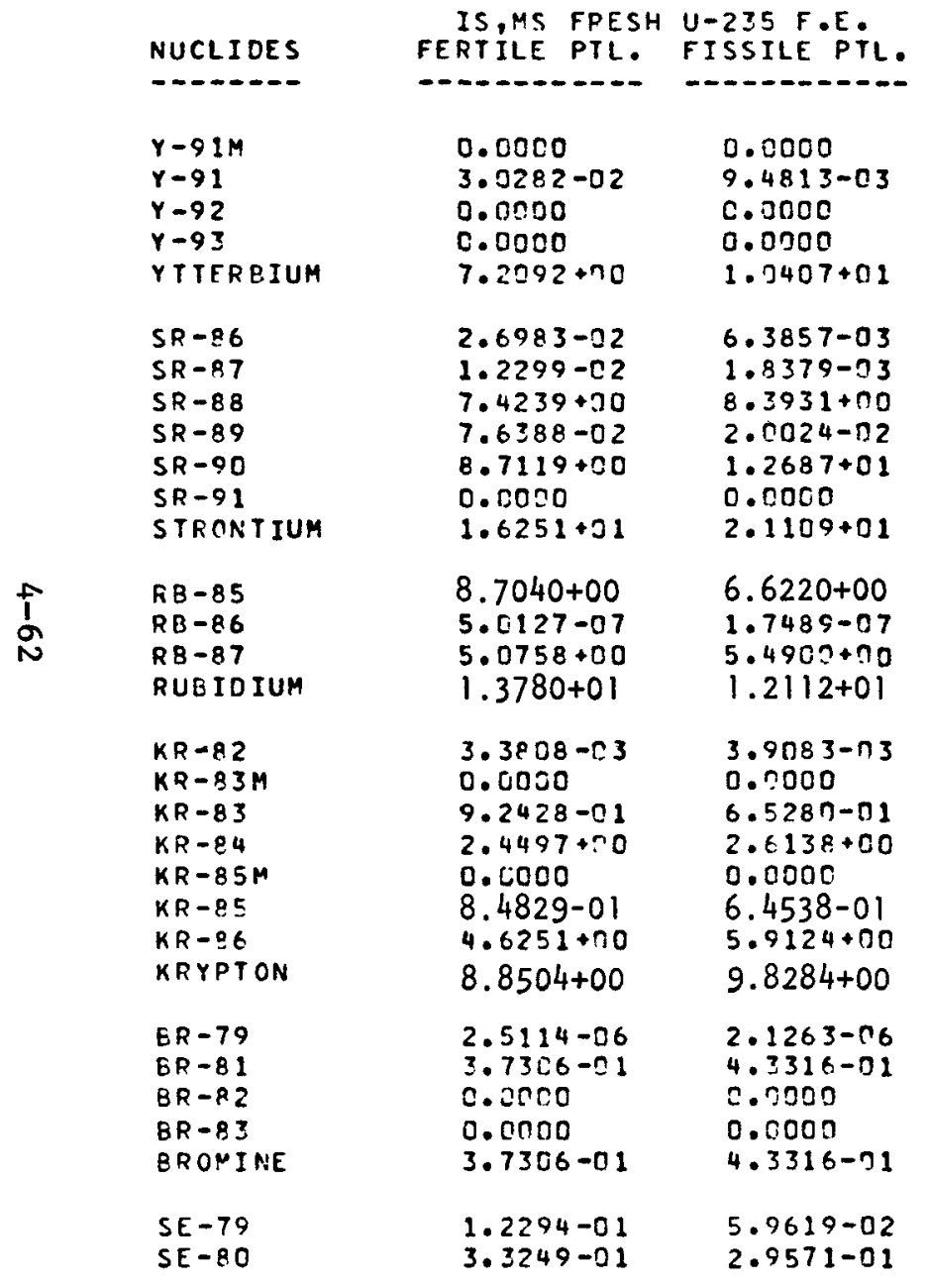

23RS RECYCLE U-233 F.E. 25RS RECYCLE U-235 F. EA FERTILE PTL. FISSILE PTL. FERTILE PTL. FISSILE PTL. FERILE PTL FERTILE PTL

$\begin{array}{ll}0.0000 & 0.0 n 00 \\ 3.0282-02 & 1.0018-n 2 \\ 0.0000 & 0.0700 \\ 0.0000 & 0.0000 \\ 7.2092+00 & 1.1416+01 \\ & \\ 2.6983-02 & 1.9286-n 2 \\ 1.2299-02 & 2.7677-03 \\ 7.4239+00 & 1.0658+01 \\ 7.6388-02 & 2.3431-02 \\ 8.7119+00 & 1.2558+01 \\ 0.0000 & 0.0 n 00 \\ 1.6251+01 & 2.3262+01 \\ & \\ 8.7040+00 & 9.1054+00 \\ 5.0127-07 & 2.9943-07 \\ 5.0758+00 & 7.2086+00 \\ 1.3780+01 & 1.6314+01 \\ 3.3809-03 & 6.6520-03 \\ 0.0000 & 0.0000 \\ 9.2428-01 & 1.0191+00 \\ 2.4497+00 & 3.8065+00 \\ 0.0900 & 0.0000 \\ 8.4829-01 & 8.8742-01 \\ 4.6251+00 & 7.5313+00 \\ 8.8504+00 & 1.3251+01 \\ & \\ 2.5114-06 & 4.9382-06 \\ 3.7306-01 & 5.3850-01 \\ 0.0000 & 0.0200 \\ 0.0000 & 0.0000 \\ 3.7306-01 & 5.3851-01 \\ & \\ 1.2294-01 & 1.3239-01 \\ 3.3249-01 & 5.1055-01 \\ & \\ & \end{array}$

$\begin{array}{ll}0.0000 & 0.0000 \\ 3.0282-02 & 7.0094-03 \\ 0.0500 & 0.0000 \\ 0.0000 & 0.0 n 0 n \\ 7.2092+00 & 7.5737+00 \\ & \\ 2.6983-02 & 1.7163-02 \\ 1.2299-02 & 1.0446-02 \\ 7.4239+00 & 6.2109+n 0 \\ 7.6388-02 & 1.4757-02 \\ 8.7119+00 & 9.2399+n 0 \\ 0.0000 & 0.0 n 00 \\ 1.6251+01 & 1.5493+n 1 \\ 8.7040+00 & 4.8115+00 \\ 5.0127-07 & 1.2536-07 \\ 5.0758+00 & 3.9909+00 \\ 1.3780+01 & 8.8025+00\end{array}$

$3.3848-03 \quad 2.8350-03$ 0.0000

9. $2428-01$

$2.4497+00$

0.0000

8.4829-01

$4.6251+00$

$8.8504+00$

2. 5114-06

$3.7306-01$

0.0000

0.0000

2.835 D-0

0.0000

$4.7372-01$
$1.8988+00$

0.0000

4.6893-01

$4.299 ?+00$

$7.1435+00$

$3.7306-01$

$1.5393-06$

$3.1501-01$

0.0500

0.0000

1.2294-01

3.1501-01

$3.3249-01$

$4.3 r .62-02$

2. $1454-01$ 
NUCLIOE WEIGHTS IN GRAMS PER F.E. AT TIME 180.0 DAYS AFTER DISCHARGE

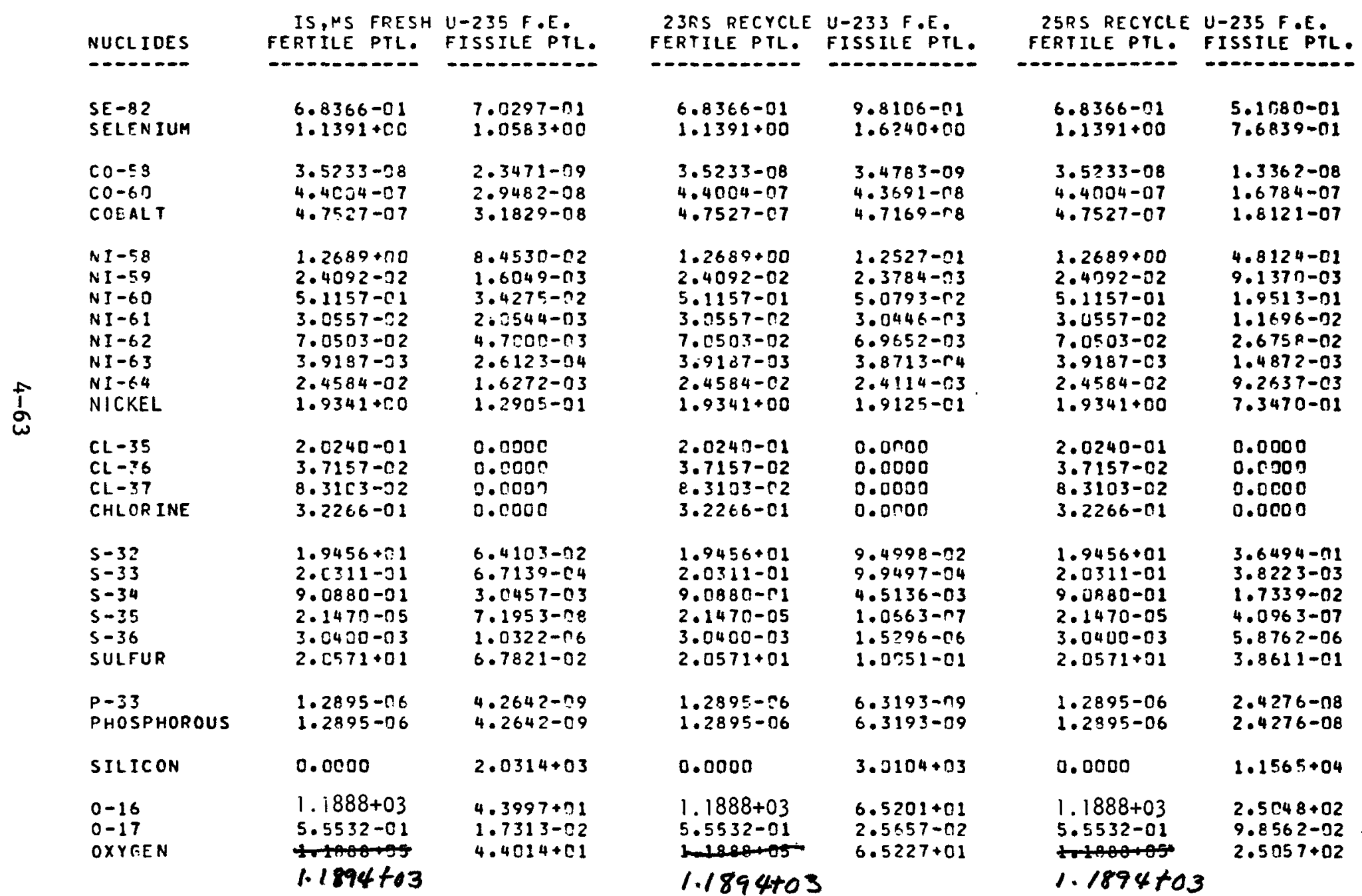


NUCLIOE HEIGHTS IN GRAMS PER F.E. AT TIME 180.0 DAYS AFTER DISCHARGE

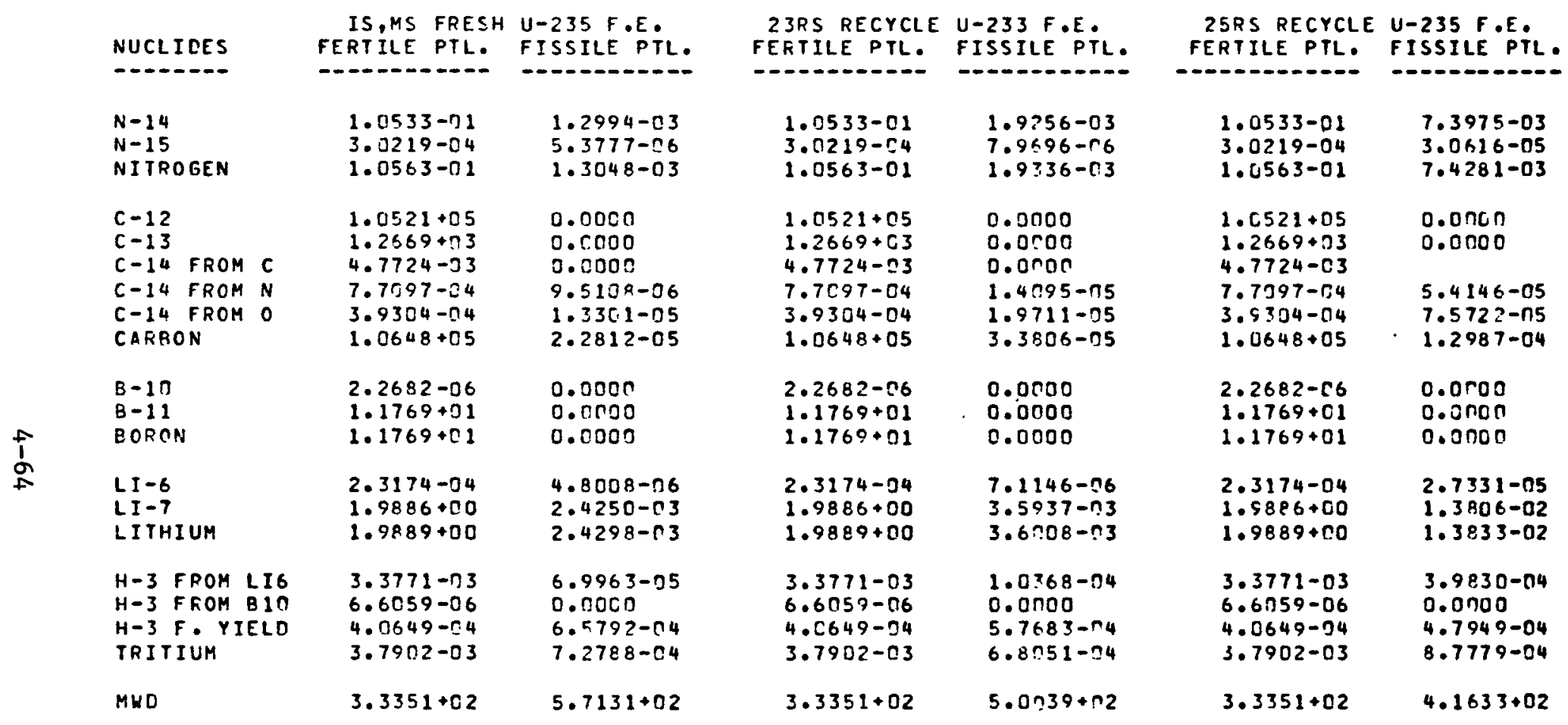


NUCLIDE ACTIVITIES IN CURIES PES F.E. AT TIME 18.0.0 DAYS AFTER DISCHARGE

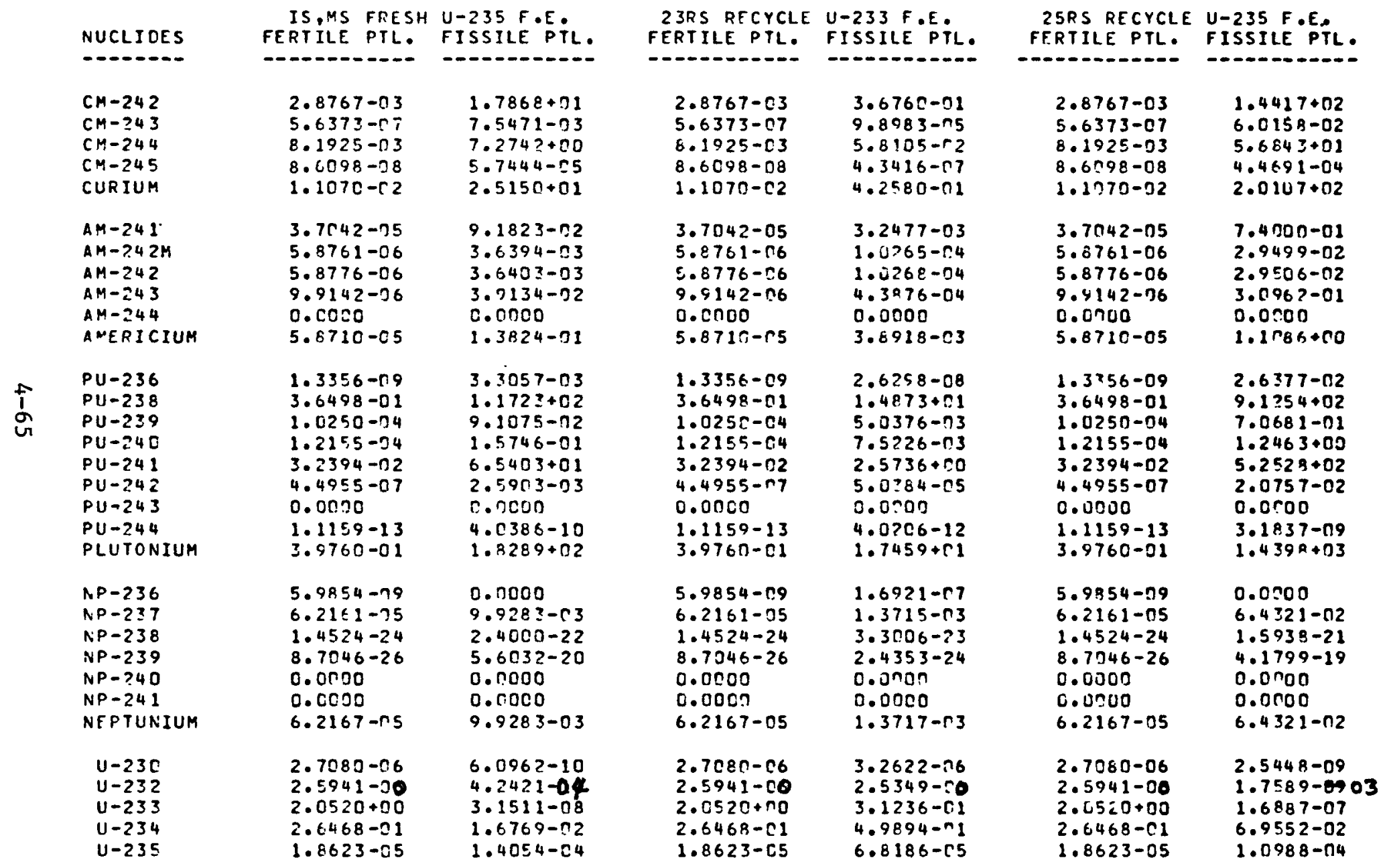


NUCLIDE ACTIVITIES IN CURIES PER F.E. AT TIME 180.0 DAYS AFTER DISCHARGE

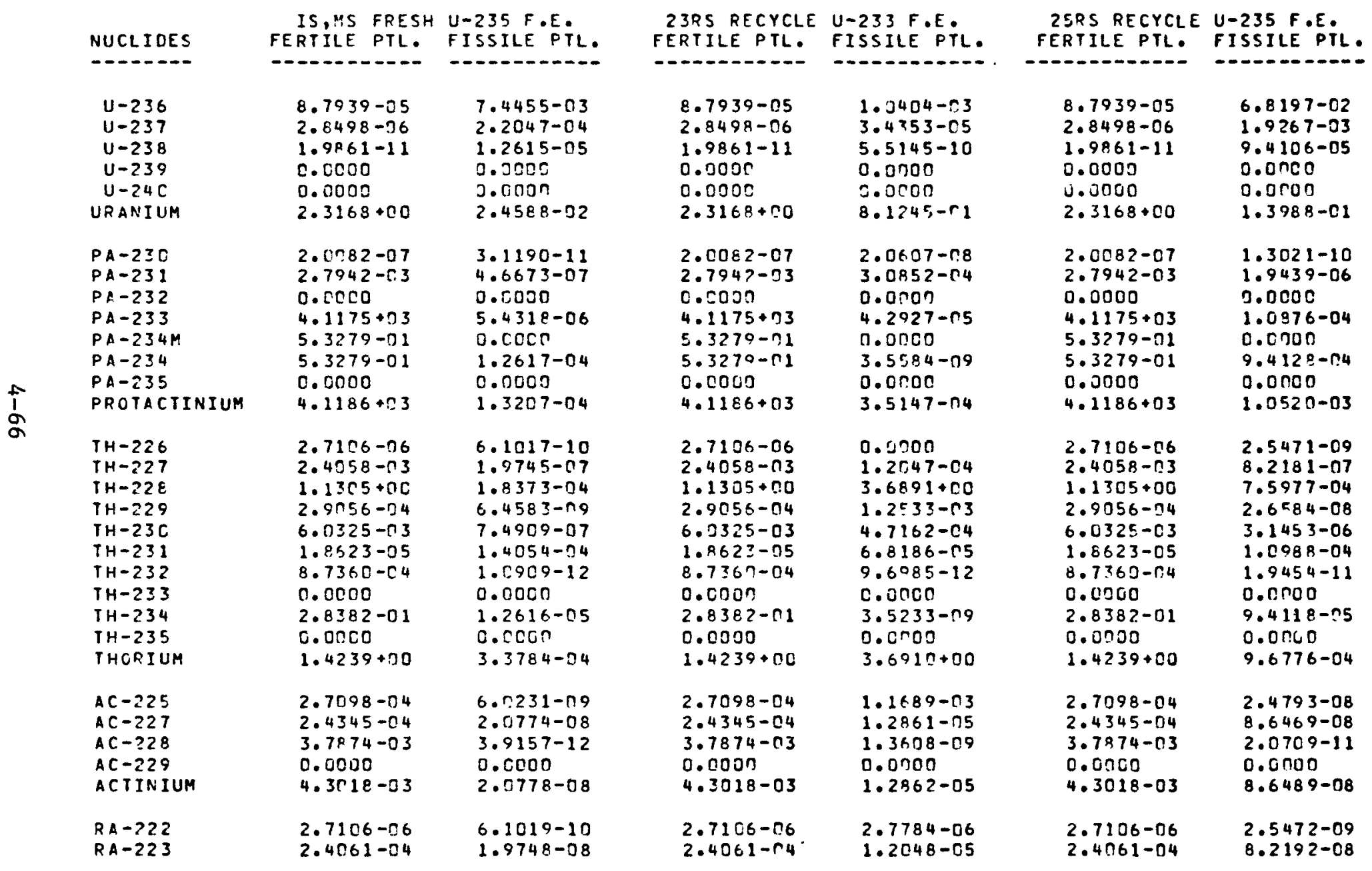


NUCLIDE ACTIVITIES IN CURIES PER F.E. AT TIME 180.0 DAYS AFTER DISCHARGE

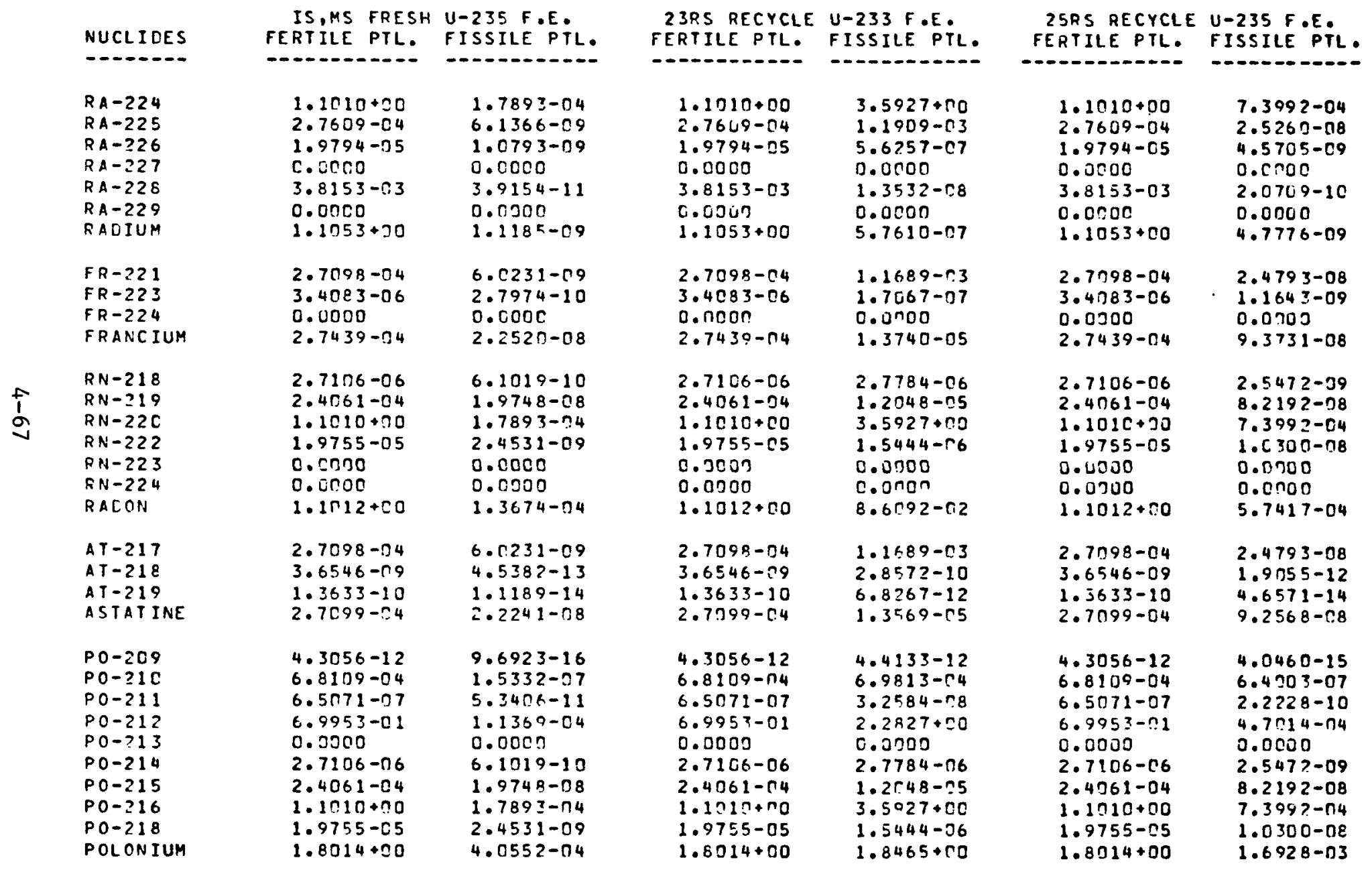


NUCLIDE ACTIVITIES IN CURIES PER F.E. AT TIME 180.0 DAYS AFTER DISCHARGE

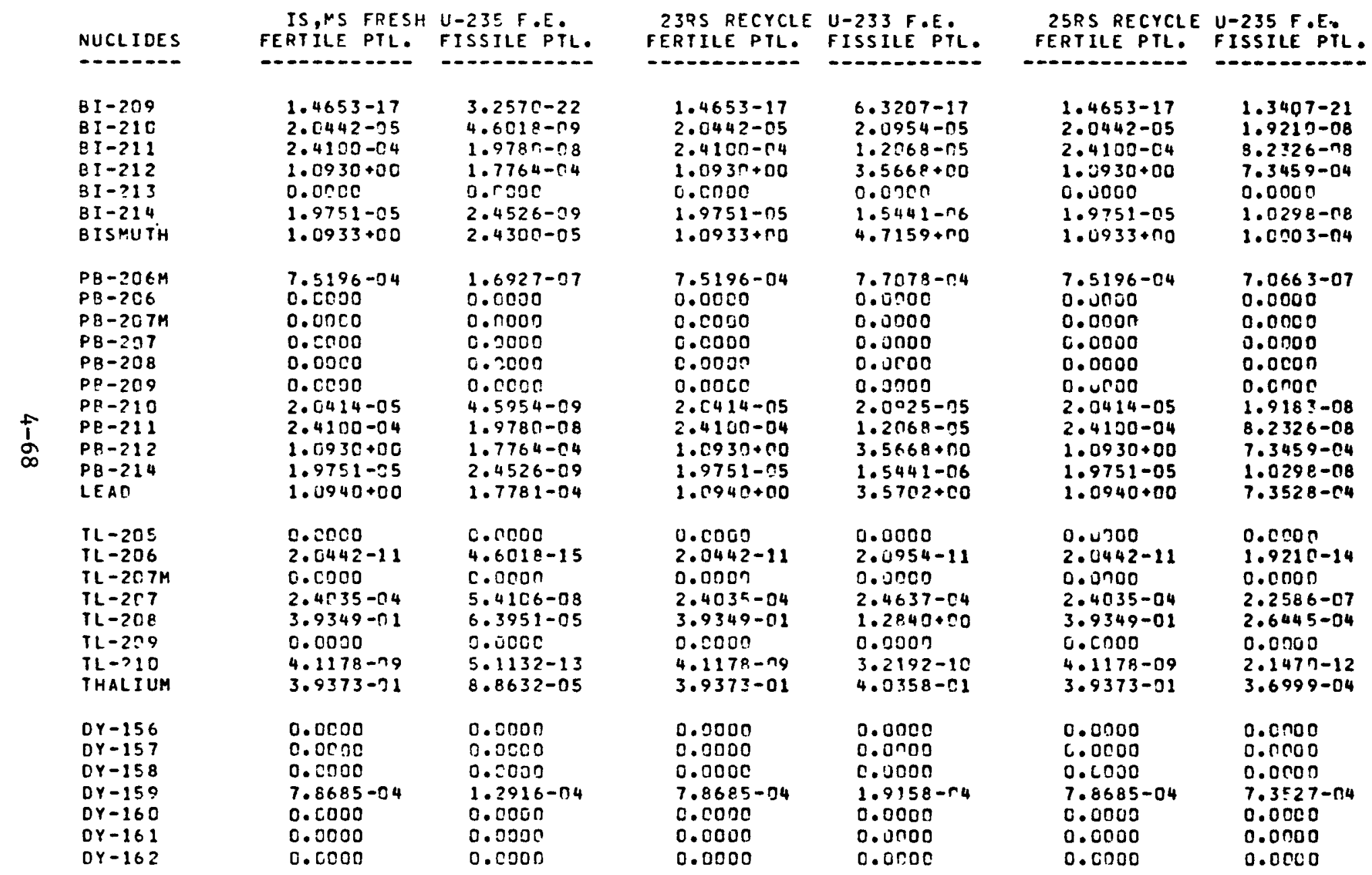


NUCLIDE ACTIVITIES IN CURIES PER F.E. AT TIME 180.0 DAYS AFTER DISCHARGE

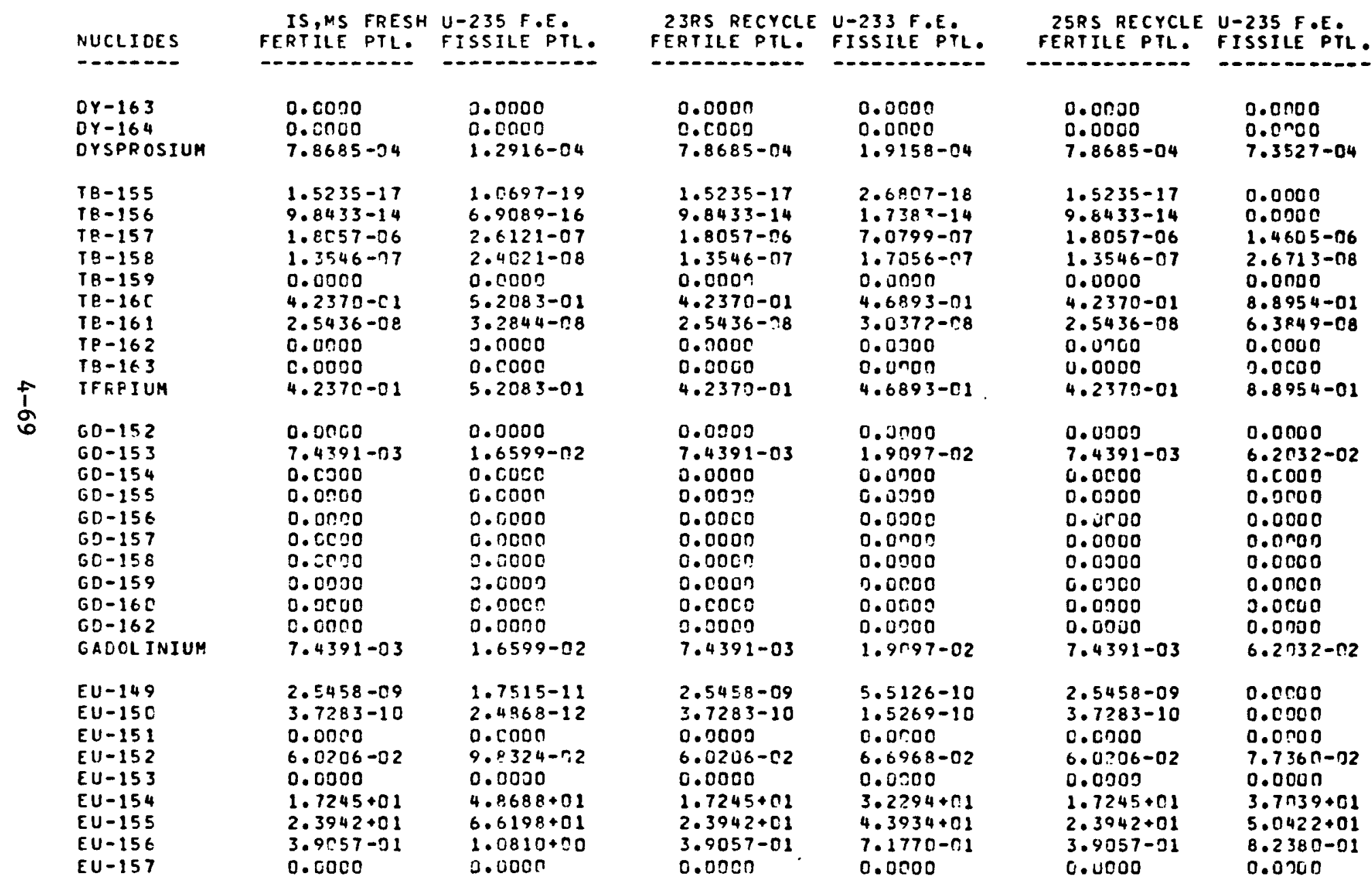


NUCLIDE ACTIVITIES IN CURIES DER F.E. AT TIME 180.0 DAYS AFTER DISCHARGE

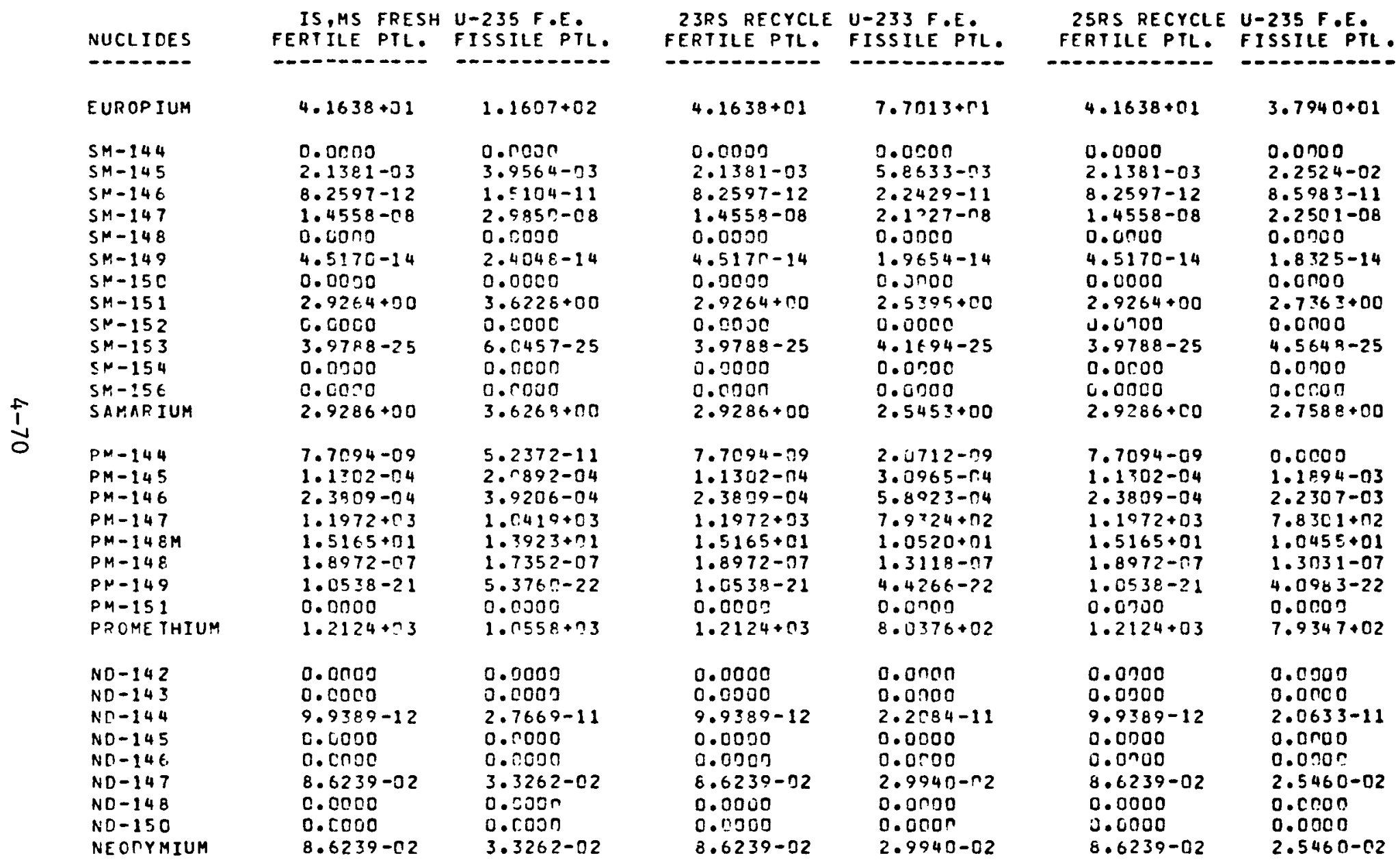


NUCLIDE ACTIVITIES IN CURIES PER F.E. AT TIME $18 \mathrm{n} .0$ DAYS AFTER OISCHARGE

\begin{tabular}{|c|c|c|c|c|c|c|}
\hline NUCLIDES & $\begin{array}{l}\text { IS,MS FRESH } \\
\text { FERTILE PTL. }\end{array}$ & $\begin{array}{l}\text { U-235 F.E. } \\
\text { FISSILE PTL. }\end{array}$ & $\begin{array}{l}\text { 23RS RFCYCLEE } \\
\text { FERTILE PTL. } \\
\end{array}$ & $\begin{array}{l}\text { U-233 F.E. } \\
\text { FISSILE PTL. }\end{array}$ & $\begin{array}{l}\text { 25RS RECYCLE } \\
\text { FERTILE PTL: }\end{array}$ & $\begin{array}{l}\text { U-235 F.E. } \\
\text { FISSILE PTL. }\end{array}$ \\
\hline $\begin{array}{l}P R-141 \\
P R-142 \\
P R-143 \\
P R-144 \\
P R-145 \\
P R A S E \text { ODYIUM }\end{array}$ & $\begin{array}{l}0.0000 \\
0.0000 \\
2.5760+00 \\
9.0979+03 \\
0.0 r 00 \\
9.0105+03\end{array}$ & $\begin{array}{l}0.0000 \\
0.0000 \\
8.0366-01 \\
8.0227+013 \\
0.0030 \\
8.0235+03\end{array}$ & $\begin{array}{l}0.0000 \\
0.0000 \\
2.5760+00 \\
9.0079+03 \\
0.0005 \\
9.0105+03\end{array}$ & $\begin{array}{l}0.0000 \\
0.0500 \\
8.1830-91 \\
6.4154+C 3 \\
0.0100 \\
6.4162+03\end{array}$ & $\begin{array}{l}0.0000 \\
0.0000 \\
2.5760+00 \\
9.0079+03 \\
0.0000 \\
9.0105+03\end{array}$ & $\begin{array}{l}0.0000 \\
0.0000 \\
6.1229-01 \\
6.0179 .03 \\
0.0107 \\
6.0186 .03\end{array}$ \\
\hline $\begin{array}{l}C E-136 \\
C E-137 \\
C E-138 \\
C E-139 \\
C E-140 \\
C E-141 \\
C E-142 \\
C E-143 \\
C E-144 \\
C E R I U M\end{array}$ & $\begin{array}{l}3.9746-11 \\
0.0000 \\
0.0000 \\
6.3587-03 \\
0.0500 \\
5.3168+02 \\
0.0 r 00 \\
0.0000 \\
8.5754+03 \\
9.1065+03\end{array}$ & $\begin{array}{l}6.5184-12 \\
0.0000 \\
0.0000 \\
7.5795-04 \\
0.0000 \\
1.5 .277+02 \\
0.0000 \\
0.0000 \\
7.6375+03 \\
7.7903+03\end{array}$ & $\begin{array}{l}3.9746-11 \\
0.0000 \\
0.0000 \\
6.3587-03 \\
0.0900 \\
5.3108+02 \\
0.0500 \\
0.0000 \\
8.5754+03 \\
9.1065+03\end{array}$ & $\begin{array}{l}9.6600-12 \\
0.0000 \\
0.0000 \\
1.6861-03 \\
0.0000 \\
1.6506+02 \\
0.0000 \\
0.0000 \\
6.1074+03 \\
6.2724+03\end{array}$ & $\begin{array}{l}3.9746-11 \\
0.0700 \\
0.0000 \\
6.3587-03 \\
0.0300 \\
5.3108+02 \\
0.0300 \\
0.0300 \\
8.5754+03 \\
9.1065+03\end{array}$ & $\begin{array}{l}3.7110-11 \\
0.0000 \\
0.0000 \\
4.2134-03 \\
0.0000 \\
1.1595+02 \\
0.7000 \\
0.0200 \\
5.7 ? 90+03 \\
5.8449+03\end{array}$ \\
\hline $\begin{array}{l}\text { LA-137 } \\
\text { LA-138 } \\
\text { LA-139 } \\
\text { LA-140 } \\
\text { LANTHANUM }\end{array}$ & $\begin{array}{l}2.4857-i 5 \\
5.7685-11 \\
0.0500 \\
1.6463+00 \\
1.6464+70\end{array}$ & $\begin{array}{l}3.2928-06 \\
1.3595-11 \\
0.0050 \\
4.7807-n 1 \\
4.7808-01\end{array}$ & $\begin{array}{l}2.4857-05 \\
5.7685-11 \\
0.0000 \\
1.6463+00 \\
1.6464+00\end{array}$ & $\begin{array}{l}1.2010-n 5 \\
2.1218-11 \\
0.0000 \\
4.9629-n 1 \\
4.9630-01\end{array}$ & $\begin{array}{l}2.4857-05 \\
5.7685-11 \\
0.0000 \\
1.6463+00 \\
1.6464+00\end{array}$ & $\begin{array}{l}1.8149-05 \\
5.200 n-11 \\
0.0000 \\
3.6440-71 \\
3.6441-01\end{array}$ \\
\hline $\begin{array}{l}B A-135 \\
B A-136 \\
B A-137 M \\
B A-137 \\
B A-138 \\
B A-14 C \\
B A R I \cup M\end{array}$ & $\begin{array}{l}0.0000 \\
0.0000 \\
1.1616+n 3 \\
0.0000 \\
0.0 r 00 \\
1.4305+00 \\
1.1631+03\end{array}$ & $\begin{array}{l}0.0000 \\
0.0000 \\
1.8027+03 \\
0.0000 \\
0.000 n \\
4.1540-01 \\
1.8032+n 3\end{array}$ & $\begin{array}{l}0.0000 \\
0.0000 \\
1.1616+03 \\
0.0000 \\
0.0000 \\
1.4305+00 \\
1.1631+03\end{array}$ & $\begin{array}{l}0.0000 \\
0.0000 \\
1.6919+03 \\
0.0000 \\
0.0200 \\
4.3122-n 1 \\
1.6923+03\end{array}$ & $\begin{array}{l}0.0000 \\
0.0000 \\
1.1616+03 \\
0.0000 \\
0.0000 \\
1.4305+00 \\
1.1631+03\end{array}$ & $\begin{array}{l}0.0000 \\
0.0000 \\
1.3447+03 \\
0.0000 \\
0.0900 \\
3.1 \in 62-01 \\
1.3450+03\end{array}$ \\
\hline $\begin{array}{l}C S-133 \\
C S-134 \\
C S-135 \\
C S-136\end{array}$ & $\begin{array}{l}0.0020 \\
1.2625+03 \\
1.8518-03 \\
7.2629-02\end{array}$ & $\begin{array}{l}0.0005 \\
3.4233+03 \\
3.6644-03 \\
5.4815-02\end{array}$ & $\begin{array}{l}0.0000 \\
1.2625+03 \\
1.8518-03 \\
7.2629-02\end{array}$ & $\begin{array}{l}0.0300 \\
2.6971+n 3 \\
2.8004-03 \\
4.9658-\Gamma 2\end{array}$ & $\begin{array}{l}0.0700 \\
1.2625+03 \\
1.8518-03 \\
7.2629-n 2\end{array}$ & $\begin{array}{l}0.0300 \\
2.5526003 \\
2.7360-03 \\
4.0701-02\end{array}$ \\
\hline
\end{tabular}


NUCLIDE ACTIVITIES IN CURIES PER F.E. AT TIME 180.0 DAYS AFTER DISCHARGE

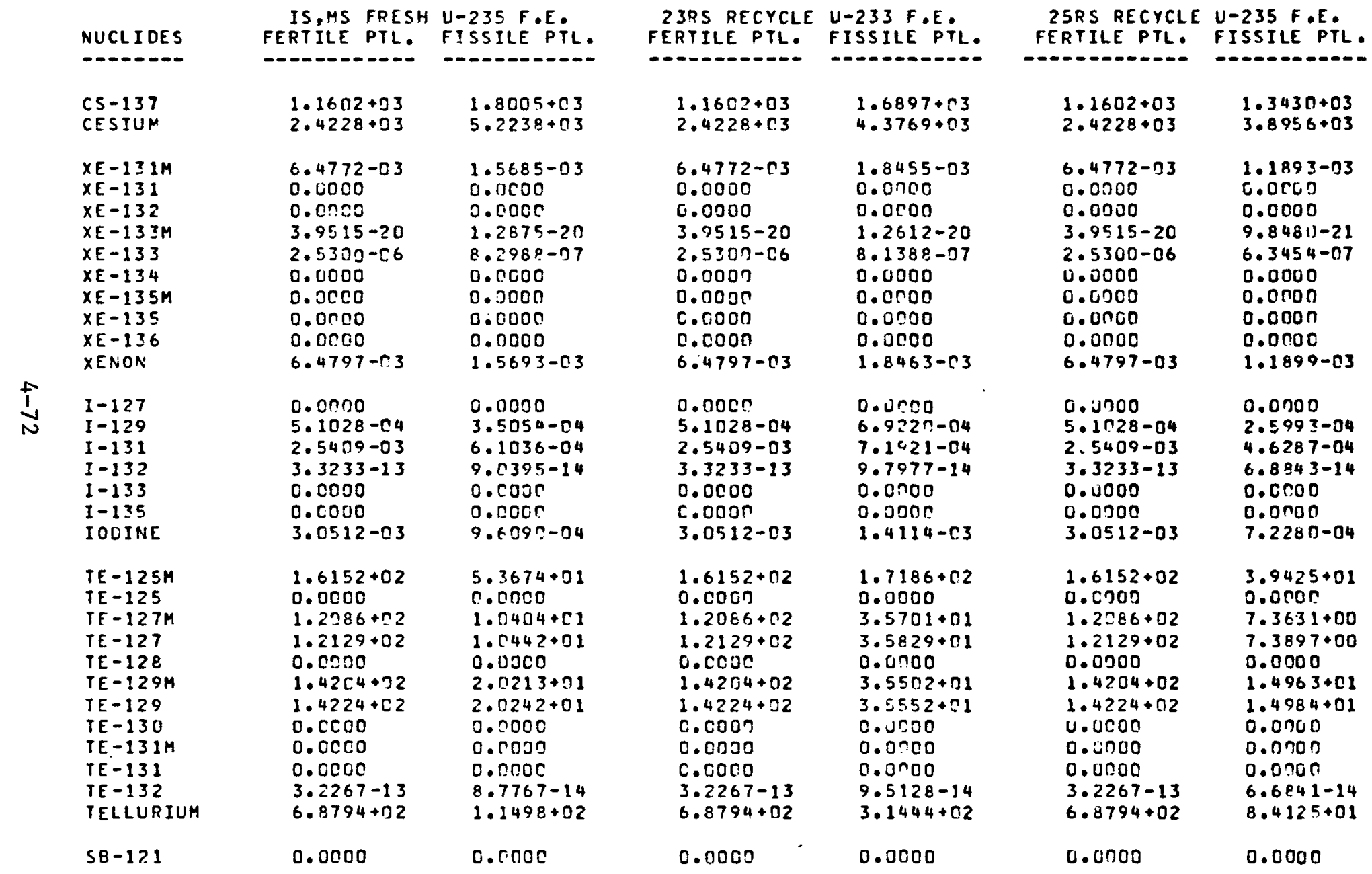


NUCLIDE ACTIVITIES IN CURIES PER F.E. AT TIME 180.0 DAYS AFTER DISCHARGE

\begin{tabular}{|c|c|c|c|c|c|c|}
\hline NUCLIDES & $\begin{array}{l}\text { IS,MS FRESH } \\
\text { FERTILE PTL. }\end{array}$ & $\begin{array}{l}\text { U-2ZS F.E. } \\
\text { FISSILE PTL. }\end{array}$ & $\begin{array}{l}23 R S \text { RECYCLE } \\
\text { FERTILE PTL. }\end{array}$ & $\begin{array}{l}\text { U-233 F.E. } \\
\text { FISSILE PTL. }\end{array}$ & $\begin{array}{l}\text { 25RS RECYCLE } \\
\text { FERTILE PTL. }\end{array}$ & $\begin{array}{l}\text { U-235 F.E. } \\
\text { FISSILE PIL. }\end{array}$ \\
\hline $\begin{array}{l}S B-122 \\
S B-123 \\
S P-124 \\
S B-125 \\
S B-127 \\
S B-128 \\
S B-129 \\
\text { ANTIMONY }\end{array}$ & $\begin{array}{l}9.9156-21 \\
0.0000 \\
1.2756-31 \\
2.5519+22 \\
2.6283-11 \\
0.0000 \\
0.5000 \\
1.5532+02\end{array}$ & $\begin{array}{l}8.1628-21 \\
0.0000 \\
9.6348-02 \\
5.0757+31 \\
1.6618-12 \\
0.000 n \\
0.0000 \\
5.0853+01\end{array}$ & $\begin{array}{l}9.9156-21 \\
0.0000 \\
1.2756-01 \\
1.5519+02 \\
2.6283-11 \\
0.0000 \\
0.0000 \\
1.5532+72\end{array}$ & $\begin{array}{l}8.1777-21 \\
0.0000 \\
1.5769-.21 \\
1.6238+C 2 \\
5.2826-12 \\
0.0700 \\
0.0000 \\
1.6254+02\end{array}$ & $\begin{array}{l}9.9156-21 \\
0.0000 \\
1.2756-01 \\
1.5519+02 \\
2.6283-11 \\
0.0000 \\
0.0000 \\
1.5532+02\end{array}$ & $\begin{array}{l}9.9453-21 \\
0.0000 \\
1.0071-01 \\
3.7277+01 \\
1.1549-12 \\
0.0900 \\
0.0000 \\
3.7378+01\end{array}$ \\
\hline $\begin{array}{l}S N-115 \\
S N-117 M \\
S N-117 \\
S N-119 M \\
S N-119 \\
S N-12 C \\
S N-121 M \\
S N-121 \\
S N-123 \\
S N-125 \\
T 1 N\end{array}$ & $\begin{array}{l}0.0000 \\
1.4926-33 \\
0.0000 \\
1.0772+00 \\
0.0000 \\
0.0050 \\
7.4790-05 \\
0.0000 \\
2.0154+01 \\
3.8898-04 \\
2.1233+01\end{array}$ & $\begin{array}{l}0.0000 \\
4.3187-04 \\
0.0000 \\
6.9598-01 \\
0.2000 \\
0.0000 \\
8.0650-05 \\
0.0000 \\
4.6189+00 \\
3.0493-05 \\
5.3154+00\end{array}$ & $\begin{array}{l}0.0000 \\
1.4926-03 \\
0.0000 \\
1.0772+00 \\
0.0000 \\
0.0000 \\
7.4790-05 \\
0.0000 \\
2.0154+01 \\
3.8898-04 \\
2.1233+01\end{array}$ & $\begin{array}{l}0.0000 \\
4.5449-04 \\
0.0000 \\
6.7570-01 \\
0.0000 \\
0.0000 \\
8.2020-05 \\
0.0000 \\
7.2986+00 \\
8.1247-75 \\
7.9749+50\end{array}$ & $\begin{array}{l}0.0000 \\
1.4926-03 \\
0.0000 \\
1.0772+00 \\
0.0000 \\
0.0000 \\
7.4790-05 \\
0.0000 \\
2.0154+01 \\
3.8298-04 \\
2.1233+01\end{array}$ & $\begin{array}{l}0.0 n 00 \\
3.2909-04 \\
0.0000 \\
5.2142-01 \\
0.0000 \\
0.0 n 00 \\
9.4953-05 \\
0.0000 \\
3.4345+100 \\
2.1695-05 \\
3.9563+100\end{array}$ \\
\hline $\begin{array}{l}\text { IN-1113 } \\
\text { IN-1 } 114 \\
\text { IN-11 } \\
\text { IN-1115 } \\
\text { INDIUM }\end{array}$ & $\begin{array}{l}0.0000 \\
4.2749-01 \\
2.6639-23 \\
0.0000 \\
4.2749-01\end{array}$ & $\begin{array}{l}0.0000 \\
6.3578-03 \\
8.1684-24 \\
0.0000 \\
6.3578-03\end{array}$ & $\begin{array}{l}0.0000 \\
4.2749-01 \\
2.6639-23 \\
0.0000 \\
4.2749-01\end{array}$ & $\begin{array}{l}0.0 ก 00 \\
9.4221-03 \\
8.7351-24 \\
0.0000 \\
9.4 .21-03\end{array}$ & $\begin{array}{l}0.0300 \\
4.2749-01 \\
2.6639-23 \\
0.0709 \\
4.2749-01\end{array}$ & $\begin{array}{l}0.0500 \\
3.6196-02 \\
6.2 \times 85-24 \\
0.0000 \\
3.6196-02\end{array}$ \\
\hline $\begin{array}{l}C D-11 C \\
C D-111 \\
C D-1112 \\
C D-1113 \\
C D-114 \\
C D-115 M \\
C D-115 \\
C A L M I \cup M\end{array}$ & $\begin{array}{l}0.0000 \\
0.0000 \\
0.0000 \\
0.0000 \\
0.0000 \\
1.1351-01 \\
2.4403-23 \\
1.1351-01\end{array}$ & $\begin{array}{l}0.0000 \\
0.0000 \\
0.0050 \\
0.0000 \\
0.0000 \\
4.5530-02 \\
7.5013-24 \\
4.5530-02\end{array}$ & $\begin{array}{l}0.0000 \\
0.0000 \\
0.0000 \\
0.0000 \\
0.0000 \\
1.1351-91 \\
2.4403-23 \\
1.1351-01\end{array}$ & $\begin{array}{l}0.0000 \\
0.0500 \\
0.0700 \\
0.0000 \\
0.0200 \\
4.6564-02 \\
8.0021-24 \\
4.6564-02\end{array}$ & $\begin{array}{l}0.0900 \\
0.0000 \\
0.0900 \\
0.0000 \\
0.0000 \\
1.1351-01 \\
2.4403-23 \\
1.1351-01\end{array}$ & $\begin{array}{l}0.0000 \\
0.0000 \\
0.0000 \\
0.0000 \\
0.0000 \\
3.4653-02 \\
5.7153-24 \\
3.4653-02\end{array}$ \\
\hline
\end{tabular}


NUCLIDE ACTIVITIES IN CURIES DER F.E. AT TIME 180.0 DAYS AFTER DISCHARGE

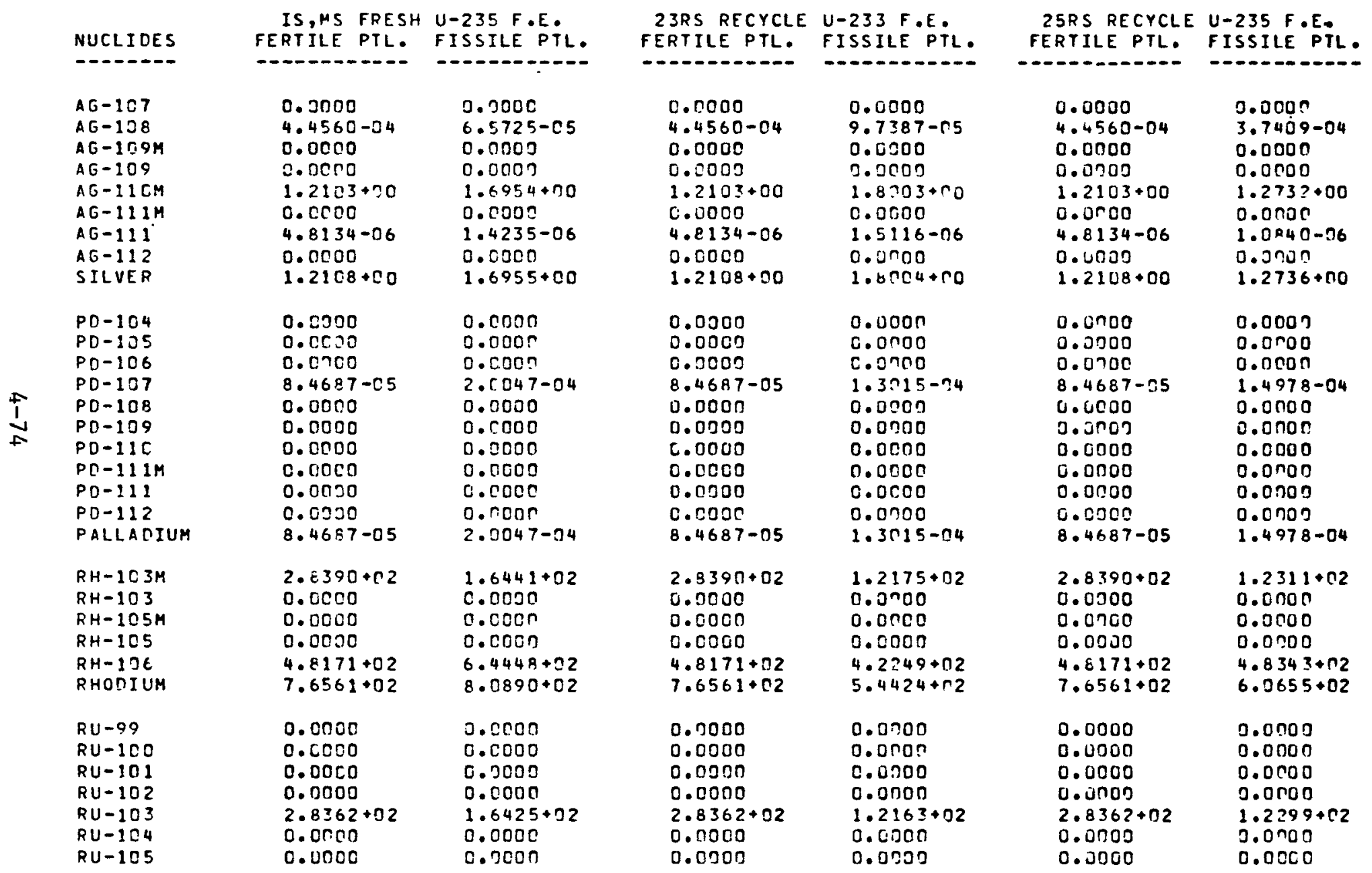


NUCLIDE ACTIVITIES IN CURIES PEP F.E. AT TIME 180.0 DAYS AFTER DISCHARGE

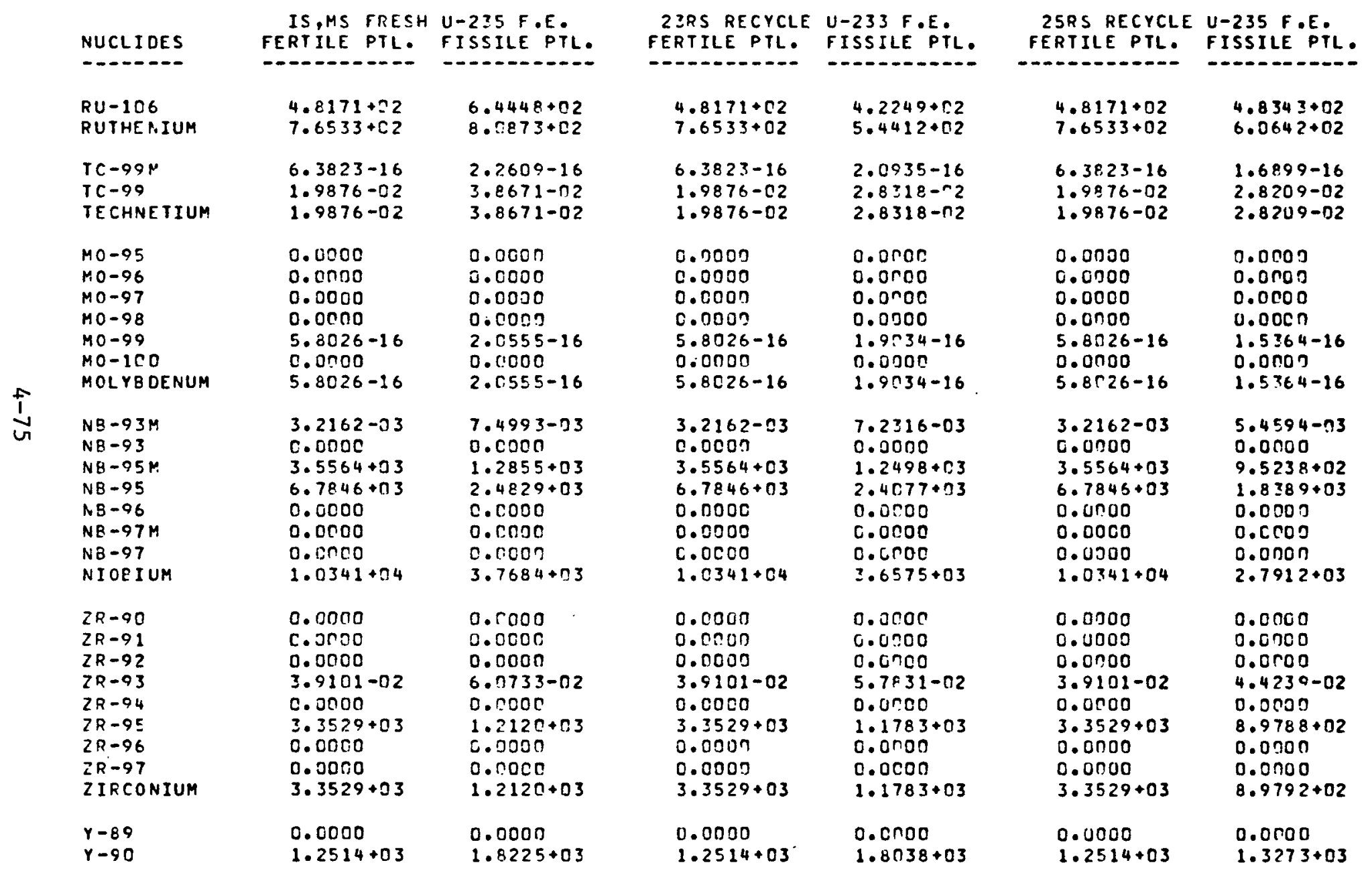


NUCLIDE ACTIVITIES IN CURIES PER F.E. AT TIME 180.0 DAYS AFTER OISCHARGE

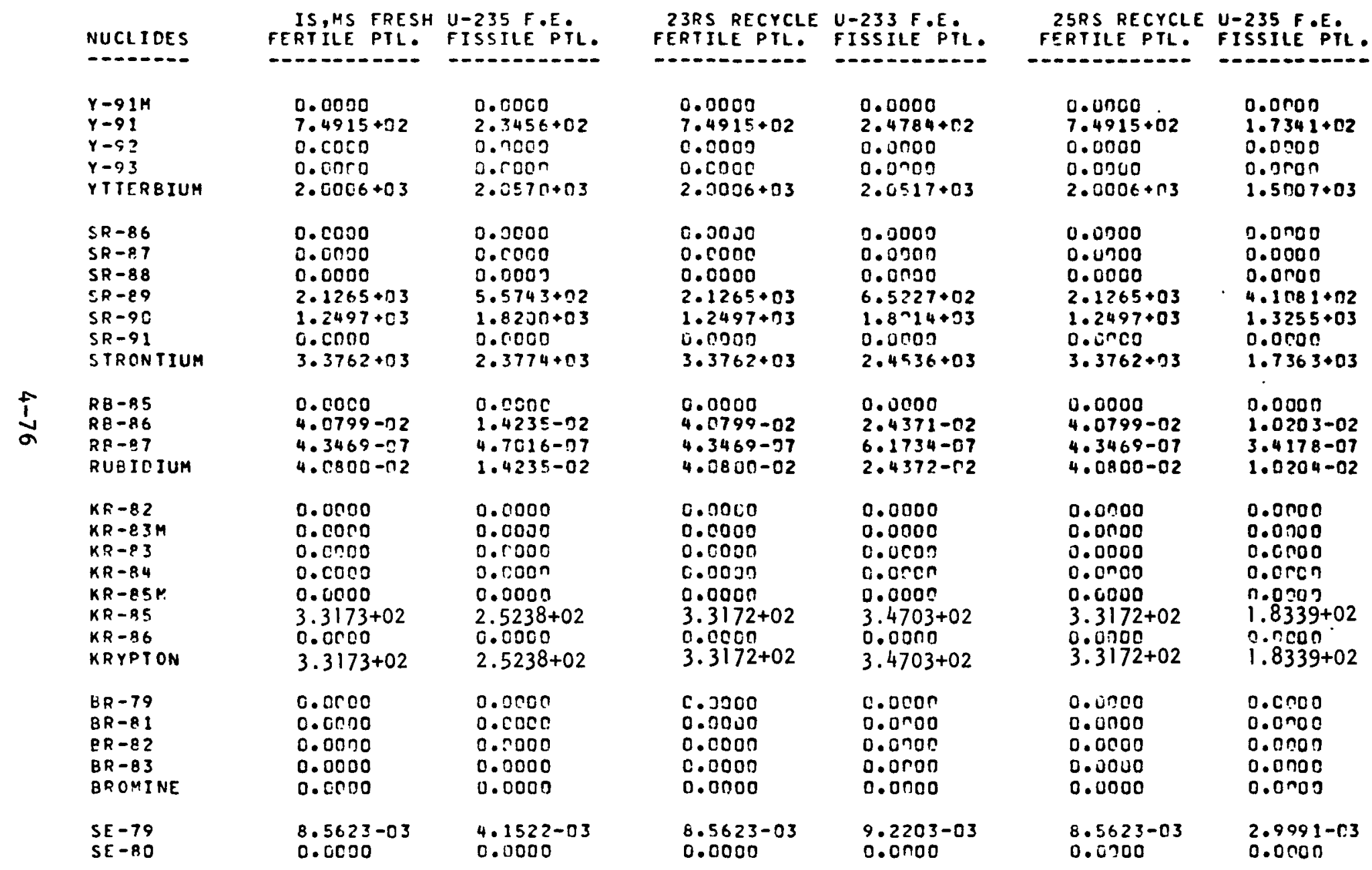


NUCLIDE ACTIVITIES IN CURIES PER F.E. AT TIME 180.C DAYS AFTER DISCHARGE

\begin{tabular}{|c|c|c|c|c|c|c|}
\hline NUCLIDES & $\begin{array}{l}\text { IS,MS FRESH } \\
\text { FERTILE PTL. }\end{array}$ & $\begin{array}{l}\text { U-235 F.E. } \\
\text { FISSILE PTL. }\end{array}$ & $\begin{array}{l}23 R S \text { RECYCLE } \\
\text { FERTILE PTL. }\end{array}$ & $\begin{array}{l}\text { U-233 F.E. } \\
\text { FISSILE PTL. }\end{array}$ & $\begin{array}{l}\text { 25RS RECYCLE } \\
\text { FERTILE PTL. }\end{array}$ & $\begin{array}{l}\text { U-235 F.E. } \\
\text { FISSILE PTL. }\end{array}$ \\
\hline$\cdots-\cdots--$ & $--0-0-0-0$ & $-\infty-0-0-0-0$ & $\cdots--0-0-1$ & 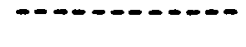 & - - n- & $---0-0-0-0-0$ \\
\hline $\begin{array}{l}\text { SE-82 } \\
\text { SELENIUM }\end{array}$ & $\begin{array}{l}0.0000 \\
8.5623-93\end{array}$ & $\begin{array}{l}0.0000 \\
4.152 ?-33\end{array}$ & $\begin{array}{l}0.000 n \\
8.5623-03\end{array}$ & $\begin{array}{l}0.0000 \\
9.2203-03\end{array}$ & $\begin{array}{l}0.0300 \\
8.5623-03\end{array}$ & $\begin{array}{l}0.0000 \\
2.9991-03\end{array}$ \\
\hline $\begin{array}{l}\text { CO-58 } \\
\text { CO-6C } \\
\text { COEALT }\end{array}$ & $\begin{array}{l}1.1115-03 \\
4.9879-04 \\
1.6103-03\end{array}$ & $\begin{array}{l}7.4042-05 \\
3.3419-75 \\
1.0746-04\end{array}$ & $\begin{array}{l}1.1115-03 \\
4.9879-04 \\
1.6103-03\end{array}$ & $\begin{array}{l}1.0973-r 4 \\
4.9525-C 5 \\
1.5925-r 4\end{array}$ & $\begin{array}{l}1.1115-03 \\
4.9879-04 \\
1.6103-03\end{array}$ & $\begin{array}{l}4.2153-04 \\
1.9025-04 \\
6.1178-04\end{array}$ \\
\hline $\begin{array}{l}N I-58 \\
N I-59 \\
N I-60 \\
N I-61 \\
N I-62 \\
N I-63 \\
N I-64 \\
N I C K E L\end{array}$ & $\begin{array}{l}0.0000 \\
1.8253-03 \\
0.0000 \\
0.0000 \\
0.0000 \\
2.4179-01 \\
0.0000 \\
2.4362-01\end{array}$ & $\begin{array}{l}0.0000 \\
1.2160-04 \\
0.0000 \\
0.0000 \\
0.0000 \\
1.61119-02 \\
0.0000 \\
1.6240-02\end{array}$ & $\begin{array}{l}0.0000 \\
1.8253-03 \\
0.3000 \\
0.0000 \\
0.0000 \\
2.4179-01 \\
0.5050 \\
2.4362-01\end{array}$ & $\begin{array}{l}0.0700 \\
1.8020-04 \\
0.0000 \\
0.0000 \\
0.0000 \\
2.3087-02 \\
0.0000 \\
2.4067-02\end{array}$ & $\begin{array}{l}0.0000 \\
1.8253-03 \\
0.0300 \\
0.0000 \\
0.0700 \\
2.4179-01 \\
0.0200 \\
2.4362-01\end{array}$ & $\begin{array}{l}0.0000 \\
6.9225-04 \\
0.0000 \\
0.0000 \\
0.0000 \\
9.1764-02 \\
0.0700 \\
9.2456-02\end{array}$ \\
\hline $\begin{array}{l}C L=35 \\
C L-36 \\
C L=37 \\
C H L O R \text { INE }\end{array}$ & $\begin{array}{l}0.0000 \\
1.2 \cos -03 \\
0 . \operatorname{con} 2 \\
1.2023-03\end{array}$ & $\begin{array}{l}0.0000 \\
0.0000 \\
0.0000 \\
0.0000\end{array}$ & $\begin{array}{l}0.300 n \\
1.2023-03 \\
0.0002 \\
1.2023-03\end{array}$ & $\begin{array}{l}0.0000 \\
0.0000 \\
0.0000 \\
0.3500\end{array}$ & $\begin{array}{l}0.0000 \\
1.2023-03 \\
0.0000 \\
1.2023-03\end{array}$ & $\begin{array}{l}0.0 n 00 \\
0.0000 \\
0.0 \text { noo } \\
0.0 \text { no }\end{array}$ \\
\hline $\begin{array}{l}s-32 \\
s-33 \\
s-34 \\
s-35 \\
s-36 \\
S U L F U R\end{array}$ & $\begin{array}{l}0.0000 \\
0.0000 \\
0.0000 \\
9.1877-21 \\
0.0030 \\
9.1877-01\end{array}$ & $\begin{array}{l}0.0000 \\
0.0000 \\
0.0000 \\
3.2791-03 \\
0.2000 \\
3.0791-03\end{array}$ & $\begin{array}{l}0.0000 \\
0.0000 \\
0.0000 \\
9.1877-01 \\
0.0000 \\
9.1877-01\end{array}$ & $\begin{array}{l}0.0000 \\
0.0000 \\
0.0000 \\
4.5631-03 \\
0.4000 \\
4.5631-03\end{array}$ & $\begin{array}{l}0.0000 \\
0.0000 \\
0.0000 \\
9.1877-01 \\
0.0000 \\
9.1877-01\end{array}$ & $\begin{array}{l}0.0700 \\
0.0500 \\
0.0000 \\
1.753 n-02 \\
0.0000 \\
1.7530-02\end{array}$ \\
\hline $\begin{array}{l}P-33 \\
\text { PHOSPHOROUS }\end{array}$ & $\begin{array}{l}0.0000 \\
0.0000\end{array}$ & $\begin{array}{l}0.3000 \\
0.0000\end{array}$ & $\begin{array}{l}0.0030 \\
0.0000\end{array}$ & $\begin{array}{l}0.0500 \\
0.0000\end{array}$ & $\begin{array}{l}0.0000 \\
0.0000\end{array}$ & $\begin{array}{l}0.0700 \\
0.0 \text { no }\end{array}$ \\
\hline SILICON & 0.0030 & 0.0000 & 0.0000 & 0.0000 & 0.0000 & 0.0000 \\
\hline $\begin{array}{l}0-16 \\
0-17 \\
\text { OXYGEN }\end{array}$ & $\begin{array}{l}0 . c 000 \\
0.0000 \\
0.0000\end{array}$ & $\begin{array}{l}0.0000 \\
0.0000 \\
0.0000\end{array}$ & $\begin{array}{l}0.0000 \\
0.0000 \\
0.0000\end{array}$ & $\begin{array}{l}0.0 n 00 \\
0.000 n \\
0.0000\end{array}$ & $\begin{array}{l}0.0000 \\
0.0000 \\
0.0000\end{array}$ & $\begin{array}{l}0.0000 \\
0.0000 \\
0.0000\end{array}$ \\
\hline
\end{tabular}


NUCLIDE ACTIVITIES IN CURIES PER F.E. AT TIME 180.0 DAYS AFTER DISCHARGE

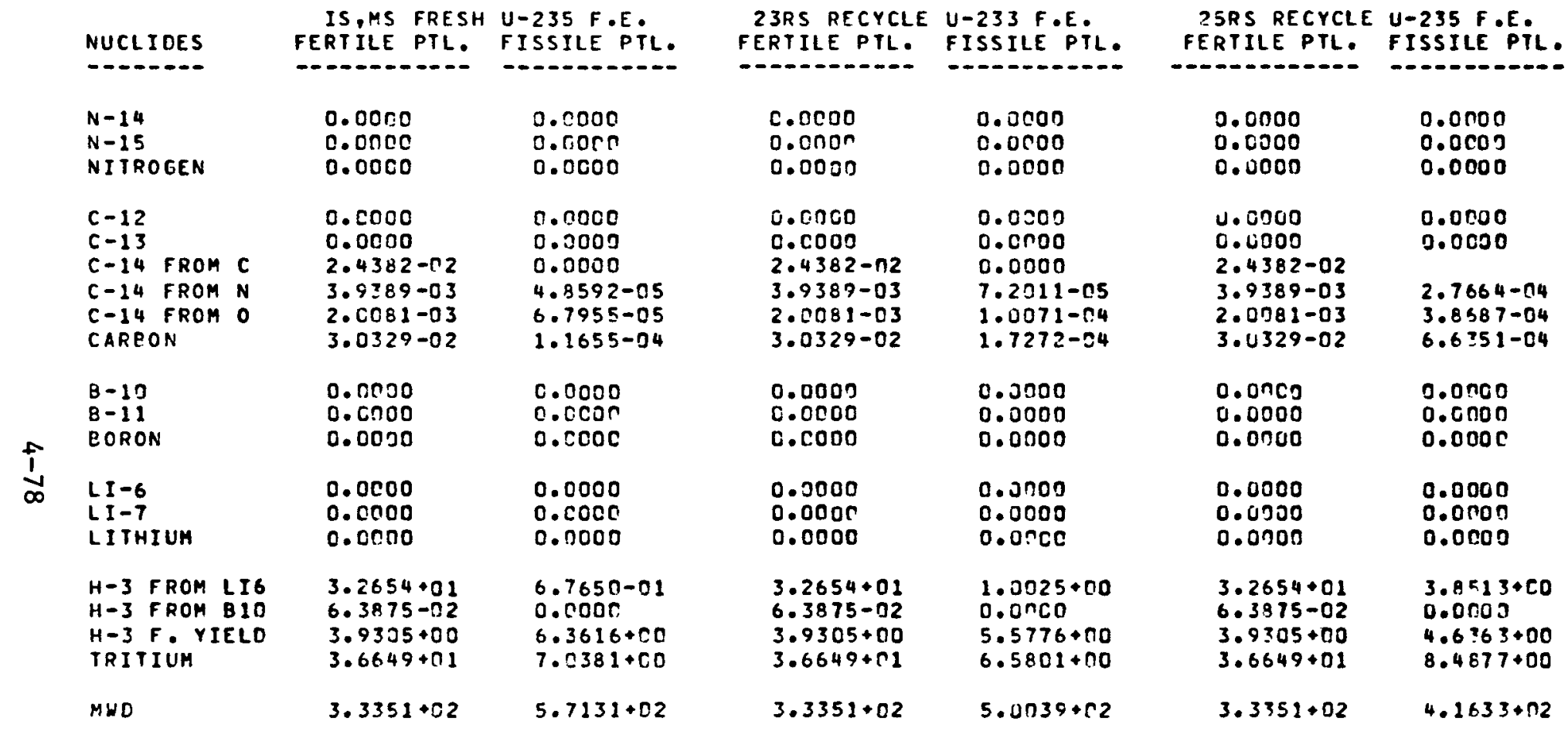




\section{CONCLUSIONS}

The HTGR discharge and reload fuel element block flow data have been forecast for an HTGR market developed by ERDA for use as a planning basis. The data illustrate the change in the number of different types of fuel elements that occurs annually for reprocessing and refabrication.

Initially, the reprocessing head end handles IS and MS elements, with the 23RS and 25RS elements entering the recycle system only after $5 \mathrm{yr}$. Throughput requirements for the U-235 PUREX and the U-233 THOREX solvent extraction systems change as the reactor market builds and as the individual reactor fuel cycles develop toward the equilibrium recycle fuel mode.

Similarly, the $25 \mathrm{R}$ and $23 \mathrm{R}$ fuel refabrication throughput requirements change with the age or development of the reactor market. Initially, about an equal number of $25 \mathrm{R}$ fuel elements and $23 \mathrm{R}$ fuel elements are refabricated. After the first year, the relative number of each type of fuel element refabricated changes until about six times as many 23R elements are refabricated as $25 \mathrm{R}$ elements.

The recycle plant reprocessing and refabrication systems will be required to have sufficient throughput flexibility to accommodate changes in the relative number of fuel element types forecast for an HTGR market.

The fuel element composition, including heavy metals, impurities, fission products, and activation products, has been calculated for reload fresh and recycle fuel elements and for discharged spent fuel elements for each operating cycle of a typical HTGR. The nuclide composition of the fissile and the fertile particles in each type of fuel element has been stored on archive tapes. 
Two codes have been written to process the fuel element composition data. The NUCLIN code reads the data tape files and computes the nuclide concentrations for user-specified decay times. Reports from printout tabulations are compiled by the LOOKUP code for the user-specified reactor operating cycles and decay periods. The LOOKUP code calculates the concentration data in units of grams per fuel element and curies per fuel element.

Although the printed results included in this volume and in Volume II are extensive, there are a number of additional cases that would be valuable for recycle fuel design engineering and analyses. Process systems, process support systems, and waste or effluent handling systems are designed for upper-limit cases. The printout results included in this report and the data available from the archive files are average data for each fuel element type. A series of calculations specifically to develop the upper limit fuel element compositions can be made for use in the design limit calculations.

As a result of ongoing fuel cycle economic studies, alternate fuel recycle strategies, reactor reload cycle variations, and fissile or fertile fuel loading variations are identified. Fuel element composition and mass flow calculations should be carried out for the promising fuel management cases.

In addition to the HTGR results presented in this report, the calculational method is applicable to other reactor or fuel composition analyses. A number of stand-in irradiated fuels are candidates for the ERDA Thorium Utilization Program hot engineering scale testing. Calculations to estimate the fuel compositions could be made to assist in the determination of the useful fuels.

With the archive data files available for the current HTGR cases or for other cases developed in the future, additional data can be derived for design uses. The calculational method should be extended to calculate 
decay heat loads as a function of decay time. Also, irradiation energies for types of decay radiation can be calculated for the nuclides present in the spent fuel. The radiation data would be necessary for shielding, activation, and dose level analyses. 


\section{REFERENCES}

1. Dahlberg, R. C., R. F. Turner, and W. V. Goeddel, "HTGR Fuel and Fuel Cycle Summary Description," General Atomic Report GA-A12801, January $21,1974$.

2. "Conceptual Design Summary and Design Qualifications for HTGR Target Recycle Plant," ERDA Report GA-A13365, General Atom1c Company, Apr11 $30,1975$.

3. "Preconceptual Design and Estimate Summary for HTGR Recycle Demonstration Facility (HRDF)," Vol. I and II, July 1975, General Atomic Company, unpublished data.

4. "Flowsheet Review for Production Reprocessing (120) and Production Refabrication (220) Requirements," ERDA Report GA-A13751, Genera1 Atomic Company, to be issued.

5. Todt, F. W., "GARGOYLE II, An Infinite Medium Fuel Cycle Analysis Code with Fuel and Poison Searches," Gulf General Atomic Report GA-9477, October 20, 1969.

6. Merril1, Meldon H., "Nuclear Design Methods and Experimental Data in Use at Gulf General Atomic," Gulf General Atomic Report Gulf-GA-A12652, July 1973.

7. "RISC Computer Code Collection ORIGEN Isotope Generation and Depletion Code - Matrix Exponential Method," contributed by Oak RIdge National Laboratory, Radlation Shielding Information Center, CCC-217, April 26, 1975.

8. Drake, M. K. (Ed.), "Data Formats and Procedure for the ENDF Neutron Cross Section Library," USAEC Report BNL-50274, Bette1le Memorial Institute, 1970.

9. Walti, P., and P. Koch, "MICROX - A Two-Region Flux Spectrum Code for the Efficient Calculation of Group Cross-Sections," Gulf General Atomic Report Gulf-GA-A10827, April 14, 1972. 
10. Bender, D. M., "Documentation of GARGOYLE Code Modifications for Extended Nuclide Chain Analysis," General Atomic Company, unpublished data.

11. "Average Nuclide Inventories in Spent HTGR Fuel," General Atomic Report, to be issued.

12. Holden, Norman E., and F. William Walker (preparers), "Chart of The Nuclides," 11th ed., revised April 1972, Knolls Atomic Power Laboratory.

13. Lederer, C. M., J. M. Hollander, and I. Perlman, Table of Isotopes, 6th ed., John Wiley \& Sons, New York, 1967.

14. Engle, G. B., and W. R. Johnson, "Properties of Unirradiated Fuel Element Graphites H-451 and TS-1240," ERDA Report GA-A13752, Genera1 Atomic Company, January 31, 1976. 